\title{
Evaluation of Cesium, Strontium, and Lead Sorption, Desorption, and Diffusion in Volcanic Tuffs from Frenchman Flat, Nevada Test Site: Macroscopic and Spectroscopic Investigations
}

\author{
prepared by \\ Charalambos Papelis and Wooyong Um \\ submitted to \\ Nevada Site Office \\ National Nuclear Security Administration \\ U.S. Department of Energy \\ Las Vegas, Nevada
}

MARCH 2003

Publication No. 45189 
Reference herein to any specific commercial product, process, or service by trade name, trademark, manufacturer, or otherwise, does not necessarily constitute or imply its endorsement, recommendation, or favoring by the United States Government or any agency thereof or its contractors or subcontractors. The views and opinions of authors expressed herein do not necessarily state or reflect those of the United States Government or any agency thereof.

This report has been reproduced directly from the best available copy.

Available for sale to the public, in paper, from:

U.S. Department of Commerce

National Technical Information Service

5285 Port Royal Rd.

Springfield, VA 22161

phone: 800.553 .6847

fax: 703.605.6000

email: order@ntis.fedworld.gov

online ordering: http://www.ntis.gov/ordering.htm

Available electronically at http://www.doe.gov/bridge

Available for a processing fee to the U.S. Department of Energy and its contractors, in paper, from:

U.S. Department of Energy

Office of Scientific and Technical Information

P.O. Box 62

Oak Ridge, TN 37831-0062

phone: 423.576 .8401

fax: 423.576.5728

email: reports@adonis.osti.gov 


\title{
Evaluation of Cesium, Strontium, and Lead Sorption, Desorption, and Diffusion in Volcanic Tuffs from Frenchman Flat, Nevada Test Site: Macroscopic and Spectroscopic Investigations
}

\author{
Prepared by \\ Charalambos Papelis and Wooyong Um \\ Division of Hydrologic Sciences \\ Desert Research Institute \\ University and Community College System of Nevada \\ Publication No. 45189 \\ Submitted to \\ Nevada Site Office \\ National Nuclear Security Administration \\ U.S. Department of Energy \\ Las Vegas, Nevada
}

March 2003

The work upon which this report is based was supported by the U.S. Department of Energy under Contract \#DE-AC08-00NV13609. Approved for public release; further dissemination unlimited. 



\section{EXECUTIVE SUMMARY}

The interaction of radionuclides and other contaminants with minerals and other aquifer materials controls the rate of migration of these contaminants in groundwater. The stronger these interactions, the more a radionuclide will be retarded. Processes such as sorption and diffusion often control the migration of inorganic compounds in aquifers. In addition, these processes are often controlled by the nature of ions of interest, the nature of the aquifer materials, and the specific geochemical conditions.

Parameters describing sorption and diffusion of radionuclides and other inorganic ions on aquifer materials are used in transport codes to predict the potential for migration of these contaminants into the accessible environment. Sorption and diffusion studies can help reduce the uncertainty of radionuclide transport modeling on the Nevada Test Site (NTS) and other nuclear testing areas. For example, reliable sorption equilibrium constants, obtained under a variety of conditions, can be used to suggest a plausible sorption mechanism and to provide retardation parameters that can be used in transport models. In addition, these experiments, performed under a variety of conditions, can lead to models that can accommodate changing geochemical conditions. Desorption studies can probe the reversibility of reactions and test whether the reversibility assumed by equilibrium models is justified. Kinetic studies can be used to probe the time-dependent limitations of reactions and suggest whether an equilibrium or kinetic model may be more appropriate. Finally, spectroscopic studies can be used to distinguish between different sorption mechanisms, and provide further guidance with respect to model selection.

Equilibrium sorption, desorption, and diffusion experiments were conducted with three different samples from Frenchman Flat on the NTS. These materials included volcanic tuff samples ER5/3/2 2800D, ER5/3/2 2960D, and ER5/3/2 3270D. The samples were characterized based on mineralogy, specific surface area, density, and porosity. Sample ER5/3/2 2800D was composed mainly of zeolites, mostly clinoptilolite, whereas sample ER5/3/2 2960D was composed mainly of feldspars and quartz with some clinoptilolite, and sample ER5/3/2 3270D was composed mainly of feldspars, quartz, and some smectites, but no zeolites.

Based on sorption data with different solid and metal concentrations, linear sorption isotherms were derived for sorption at $\mathrm{pH} 8.3$, representing a common NTS groundwater $\mathrm{pH}$. Following sorption, desorption experiments were conducted to determine the reversibility of the sorption reactions. Lead was the most reactive metal ion and exhibited considerable $\mathrm{pH}$-dependent sorption on sample 3270D. Sorption on the other two samples was much less $\mathrm{pH}$ dependent for all three cations. Typical $K_{\mathrm{d}} \mathrm{s}$ for $\mathrm{Pb}$ sorption ranged from $5.5 \times 10^{-3}$ to $1.29 \times 10^{-2} \mathrm{~m}^{3} / \mathrm{g}$. The sorption of $\mathrm{Cs}$ and $\mathrm{Sr}$ was much less $\mathrm{pH}$ dependent and was overall substantially reduced compared to $\mathrm{Pb}$. Typical $K_{\mathrm{d}} \mathrm{S}$ for Cs and $\mathrm{Sr}$ were approximately one order of magnitude less than $\mathrm{Pb} K_{\mathrm{d}} \mathrm{S}$ $\left(6.72 \times 10^{-4}\right.$ to $\left.2.78 \times 10^{-3} \mathrm{~m}^{3} / \mathrm{g}\right)$. In all but one case, $K_{\mathrm{d}} \mathrm{s}$ obtained from desorption experiments were higher than the $K_{\mathrm{d}} \mathrm{s}$ obtained from sorption experiments. The reversibility of sorption was a function of both the sorbent mineral phase and the metal cation.

Diffusion experiments were conducted with coarser particles, compared to the finer particles used in the sorption and desorption experiments. These experiments were conducted with $\mathrm{Cs}, \mathrm{Sr}$, and $\mathrm{Pb}$. The data were fitted with a pore diffusion model, assuming diffusion into a sphere from a solution of limited volume and constant concentration. The diffusion model was better at representing the uptake of cations by samples 2960D and 3270D than sample 2800D. This is because $2800 \mathrm{D}$, the high zeolite concentration sample, with its high cation exchange 
capacity, resulted in fast quantitative removal of all three cations. The uptake of these cations by $2800 \mathrm{D}$ was therefore not controlled by a diffusion process and the model results were consistent with this hypothesis.

The elemental surface composition, determined by x-ray photoelectron spectroscopy (XPS), was consistent with the known sample mineralogy. The binding energies for $\mathrm{Cs}$ and $\mathrm{Sr}$ were consistent with the expected coordination environment of these metals under these conditions. The surface coverage of adsorbents by Cs and Sr, independently determined by XPS measurements following the sorption and diffusion experiments, was compared to estimates from macroscopic uptake experiments. The XPS measurements of surface coverage agreed well with the estimates from sorption experiments.

These experiments clearly show the difference in degree of interaction between different metal ions with different aquifer materials. The spectroscopic experiments allowed distinction between different possible sorption mechanisms and helped explained observations from macroscopic sorption experiments. Finally, this work showed the significance of aquifer material properties on the observed radionuclide transport behavior and the importance of a combination of studies to reduce the uncertainties of transport modeling.

\section{ACKNOWLEDGEMENTS}

This work could not have been completed without the dedicated and careful experimental work of Rebekah Harris, Christine Riland, and Greg Schmett. Support for this work was provided by the U.S. Department of Energy under Contract \#DE-AC08-00NV13609. The continued support of Robert Bangerter is greatly appreciated. The comments of Tim Rose, Carol Bruton, Mavrik Zavarin, Janet Wille, and Dave Finnegan improved the report and are gratefully acknowledged. 


\section{TABLE OF CONTENTS}

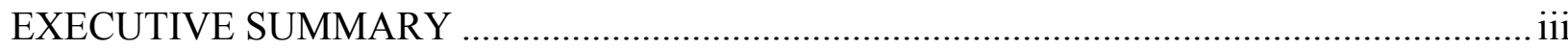

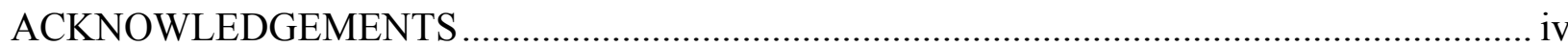

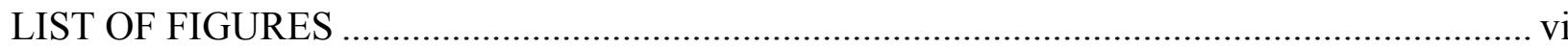

LIST OF TABLES …………………………………................................................ vii

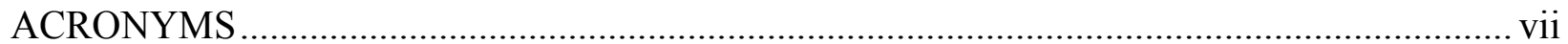

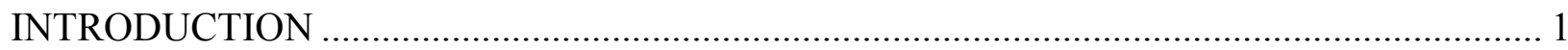

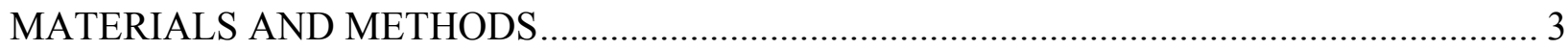

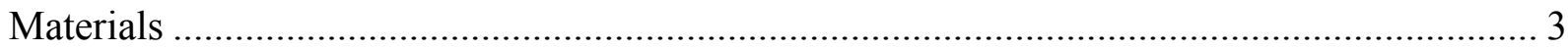

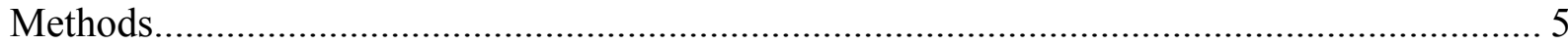

Batch Sorption/Desorption Equilibrium Experiments ......................................................... 5

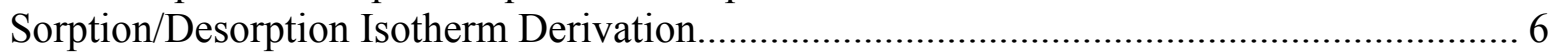

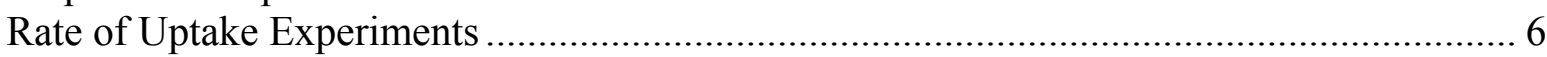

X-ray Photoelectron Spectroscopy (XPS) …………………………………………... 7

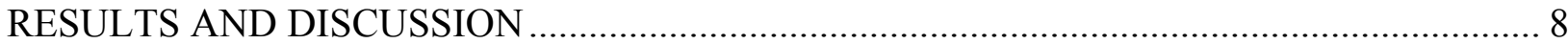

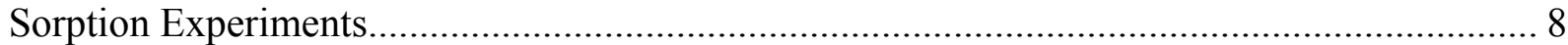

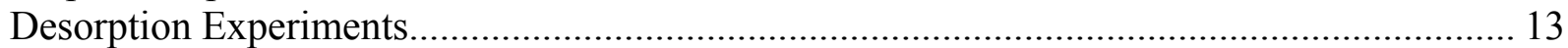

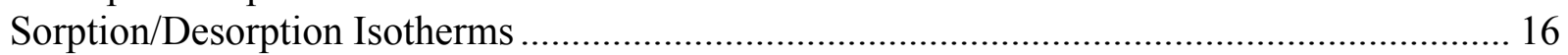

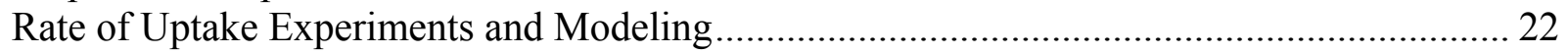

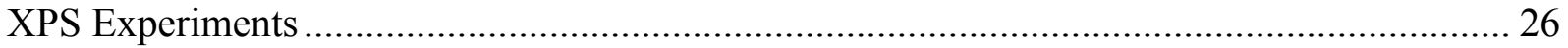

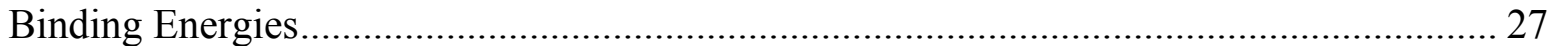

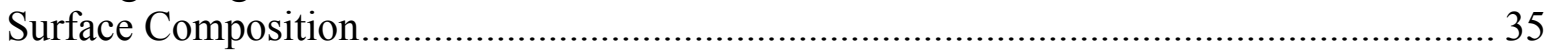

Estimation of Sorbent Surface Coverage by Cesium, Lead, and Strontium.......................... 37

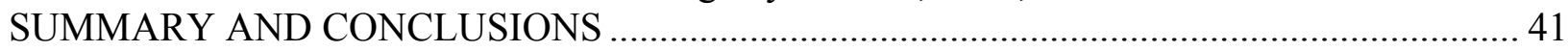

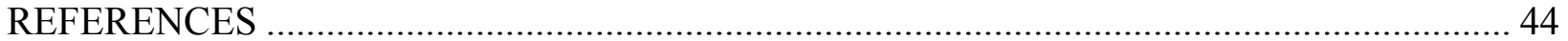




\section{LIST OF FIGURES}

1. Sorption of $10^{-5} \mathrm{M}$ Sr on $3 \mathrm{~g} / \mathrm{L}$ of samples $2800 \mathrm{D}$, 2960D, and 3270D.............................. 9

2. Sorption of $10^{-5} \mathrm{M}$ Cs on $3 \mathrm{~g} / \mathrm{L}$ of samples 2800D, 2960D, and 3270D........................... 10

3. Sorption of $10^{-5} \mathrm{M} \mathrm{Pb}$ on $3 \mathrm{~g} / \mathrm{L}$ of samples 2800D, 2960D, and 3270D .......................... 11

4. Sorption of $10^{-6} \mathrm{M}$ Se on $10 \mathrm{~g} / \mathrm{L}$ of samples 2800D, 2960D, and 3270D........................... 12

5. Sorption of $10^{-6} \mathrm{M} \mathrm{Cr}$ on $10 \mathrm{~g} / \mathrm{L}$ of samples 2800D, 2960D, and 3270D.......................... 13

6. Percent Sr release following sorption of $10^{-5} \mathrm{M} \mathrm{Sr}$ on $3 \mathrm{~g} / \mathrm{L}$ of samples $2800 \mathrm{D}, 2960 \mathrm{D}$, and 3270D.

7. Percent Cs release following sorption of $10^{-5} \mathrm{M}$ Cs on $3 \mathrm{~g} / \mathrm{L}$ of samples $2800 \mathrm{D}, 2960 \mathrm{D}$,

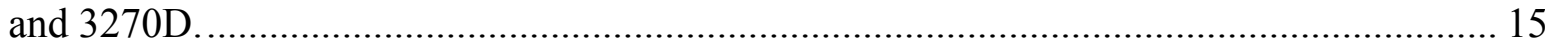

8. Percent $\mathrm{Pb}$ release following sorption of $10^{-5} \mathrm{M} \mathrm{Pb}$ on $3 \mathrm{~g} / \mathrm{L}$ of samples 2800D, 2960D,

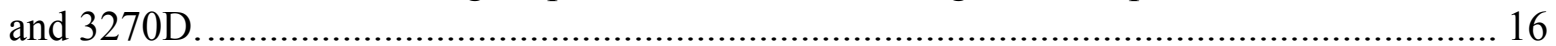

9. Linear sorption and desorption Sr isotherms for samples 2800D, 2960D, and 3270D....... 18

10. Freundlich Sr isotherms for samples 2800D, 2960D, and 3270D . ................................ 18

11. Linear sorption and desorption Cs isotherms for samples 2800D, 2960D, and 3270D........ 20

12. Freundlich Cs isotherms for samples 2800D, 2960D, and 3270D. ................................. 20

13. Linear sorption and desorption $\mathrm{Pb}$ isotherms for samples 2800D, 2960D, and 3270D........ 21

14. Freundlich $\mathrm{Pb}$ isotherms for samples 2800D, 2960D, and 3270D. ................................... 21

15. Modeling the rate of Sr uptake by samples 2800D, 2960D, and 3270D ............................. 23

16. Modeling the rate of Cs uptake by samples 2800D, 2960D, and 3270D .............................. 24

17. Modeling the rate of $\mathrm{Pb}$ uptake by sample 2800D............................................................. 25

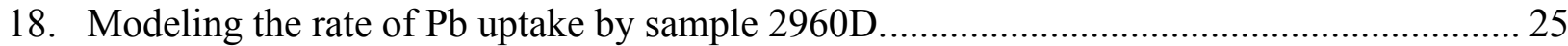

19. Modeling the rate of $\mathrm{Pb}$ uptake by sample 3270D............................................................. 26

20. Typical XPS survey scan of a Frenchman Flat powder sample.............................................2 27

21. Typical Al 2p x-ray photoelectron spectrum in a Frenchman Flat powder sample...............29

22. Typical Si $2 p x$-ray photoelectron spectrum in a Frenchman Flat powder sample. .............. 30

23. Typical O 1s x-ray photoelectron spectrum in a Frenchman Flat powder sample. ............... 30

24. Typical Na 1s x-ray photoelectron spectrum in a Frenchman Flat powder sample............... 31

25. Typical K 2p x-ray photoelectron spectrum in a Frenchman Flat powder sample................ 32

26. Typical Ca $2 \mathrm{p}$ x-ray photoelectron spectrum in a Frenchman Flat powder sample. ............. 33

27. Typical Fe 2p x-ray photoelectron spectrum in a Frenchman Flat powder sample................ 33

28. Typical $\mathrm{Sr} 3 \mathrm{~d} \mathrm{x}$-ray photoelectron spectrum in a Frenchman Flat powder sorption sample.

29. Typical Cs $3 d \mathrm{~d}$-ray photoelectron spectrum in a Frenchman Flat powder sorption sample. 


\section{LIST OF TABLES}

1. Physicochemical characteristics of Frenchman Flat samples. ....................................... 3

2. Specific surface area and pore information for different sized samples............................ 4

3. Linear sorption/desorption isotherms for $\mathrm{Sr}, \mathrm{Cs}$, and $\mathrm{Pb}$............................................ 19

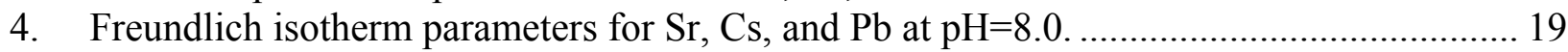

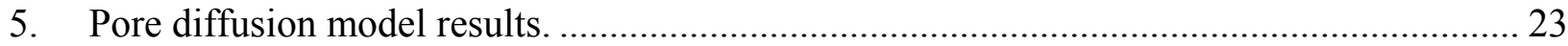

6. Chemical shifts and binding energies $(\mathrm{eV})$ of major elements in powder samples............ 28

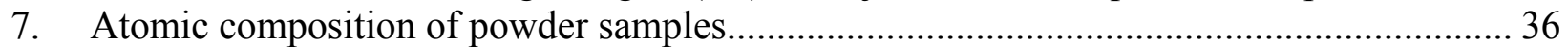

8. Comparison of percent monolayer coverage of Frenchman Flat powders as determined

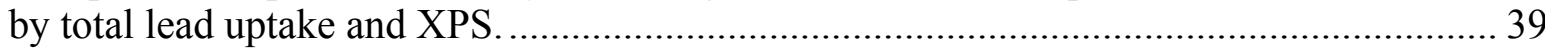

9. Comparison of percent monolayer coverage of Frenchman Flat powders as determined

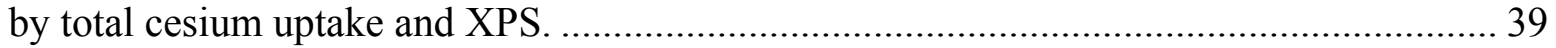

10. Comparison of percent monolayer coverage of Frenchman Flat powders as determined by total strontium uptake and XPS.

\section{ACRONYMS}

$\begin{array}{ll}\text { AA } & \text { atomic adsorption } \\ \text { BE } & \text { binding energy } \\ \text { LANL } & \text { Los Alamos National Laboratory } \\ \text { NTS } & \text { Nevada Test Site } \\ \text { XPS } & \text { x-ray photoelectron spectroscopy } \\ \text { XRD } & \text { x-ray diffraction }\end{array}$





\section{INTRODUCTION}

During the cold war, the United States government conducted a series of nuclear weapons tests at various nuclear test sites throughout the country. Nuclear weapons tests started at the Nevada Test Site (NTS) on January 27, 1951. Until 1992, more than 1,021 detonations including both atmospheric and underground tests have been conducted at different locations on the NTS (U.S. Department of Energy, 2000). Underground nuclear tests were conducted in a variety of geologic and hydrologic environments to prevent the atmospheric fallout of radioactive debris from surface detonations at the NTS (U.S. Department of Energy, 2000). However, both types of tests resulted in contamination from radionuclides as well as from other toxic metals at the surface and underground, especially in the subsurface aqueous environment.

Radionuclides included both uranium and heavy transuranic elements (man-made radionuclides heavier than uranium), such as neptunium, plutonium, and americium, as well as radioactive isotopes of lighter elements, typically fission products of uranium and plutonium. In addition, nuclear tests have released substantial amounts of non-radioactive, hazardous inorganic heavy metals, such as lead, copper, arsenic, chromium, and cadmium, and possibly organic compounds such as PVC, polystyrene, and phenols (Bryant and Fabryka-Martin, 1991). In the case of tests conducted above the groundwater table, these contaminants may potentially move downward to the groundwater table and therefore travel with the groundwater outside the NTS boundary, posing environmental health risks, because of their toxicity, radioactivity, or carcinogenicity (Sax, 1981; Chang, 1988; Francis, 1994). In other cases, tests were conducted below groundwater level, so that radionuclides and other contaminants would be available for transport following nuclear melt glass dissolution. Because of the potential health hazards associated with these contaminants, fate and transport models are being developed to predict contaminant transport for up to 1,000 years in the future.

The severity of environmental problems associated with these contaminants is related to their potential movement from the source of contamination into the accessible environment. The fate and transport of individual contaminants in the aqueous phase is largely controlled by the degree of contaminant interaction with the surrounding solid matrix. Radionuclides and metals with high sorption affinity for the surrounding mineral phases tend to be severely retarded compared to groundwater flow. Other contaminants with little sorptive affinity for the mineral surfaces present are less retarded so that they move essentially at groundwater velocity. An understanding of the potential distribution for a particular type of contaminant between the aqueous and the solid phases likely to be encountered in a given flowpath is required to predict the fate and transport of contaminants in the subsurface environment. The failure to consider sorption and desorption processes can lead to errors in estimation of the amount of a contaminant at a point some distance from the source, as well as the time required for travel.

In addition to sorption/desorption experiments used to predict the potential for migration of contaminants, studies of diffusion are also essential to fully understand the fate and transport of contaminants. Diffusion is an important transport mechanism, especially in slow groundwater flow, where the migration of solutes can be retarded by diffusion of contaminants into the solid matrix (Shackelford, 1991; Tachi et al; 1998). Although groundwater flow is relatively fast in fractures, diffusion can behave like a retarding mechanism by removing contaminants from the groundwater in fractures to the surrounding matrix, in essence resulting in contaminant dilution (Neretnieks, 1980). If the radionuclides and other contaminants sorb onto the rock matrix or react chemically with it, diffusion into the matrix will lead to sorption not only at fracture surfaces but 
also at the surfaces of micropores in the rock matrix as well (Skagius and Neretnieks, 1986). Therefore, a better understanding of sorption/desorption and diffusion processes and parameters obtained from these experiments are required to predict the migration of radionuclides and other contaminants at the NTS and other nuclear test sites.

Because interactions at mineral-water interfaces control the degree of sorption/desorption and the rate of contaminant uptake in a porous matrix, specific investigations of processes occurring at the mineral-water interface are necessary. Macroscopic and microscopic experiments provide useful information for sorption uptake, desorption release, and physicochemical properties of adsorbents. Such studies, however, cannot reveal the nature of specific interactions of individual contaminants with mineral surfaces. In this study, $\mathrm{x}$-ray photoelectron spectroscopy (XPS) was used to obtain chemical state information at mineralwater interfaces. XPS can be used to obtain chemical composition of surfaces by measuring the kinetic energy of ejected, x-ray-induced photoelectrons. The kinetic energy of the electrons gives information about the binding energy of electrons involved and the oxidation state of the specific element, as well as dissolution, precipitation, and weathering reactions at surfaces (Biino and Grönig, 1998; Hochella, 1988, 1990). Because XPS is a surface-sensitive technique, only the outermost surface layers of the adsorbents can be examined.

The present study included sorption/desorption, diffusion, and XPS experiments of divalent lead $\left(\mathrm{Pb}^{\mathrm{II}}\right)$, divalent strontium $\left(\mathrm{Sr}^{\mathrm{II}}\right)$, monovalent cesium $\left(\mathrm{Cs}^{\mathrm{I}}\right)$, hexavalent chromium, as chromate $\left(\mathrm{Cr}^{\mathrm{VI}}\right)$, and tetravalent selenium, as selenite $\left(\mathrm{Se}^{\mathrm{IV}}\right)$ ions on volcanic tuff samples from Frenchman Flat of the NTS. These particular ions were selected either because they are commonly found fission products in nuclear test sites $(\mathrm{Cs}, \mathrm{Sr})$, because they are toxic $(\mathrm{Pb}, \mathrm{Se}$, $\mathrm{Cr}$ ) or because they serve as good analogs for transport of other radionuclides (chromate and selenite were used as analogs for pertechnetate). The toxicity of $\mathrm{Pb}$ to humans has been known for over 2,000 years. The toxic effects of $\mathrm{Pb}$ on the human body are cumulative (Chang, 1988). Inhaled or ingested $\mathrm{Pb}$ concentrates in the blood, tissues, and bones in mammals and $\mathrm{Pb}$ exceeding $0.4 \mathrm{ppm}$ in human blood could cause adverse health effects (Sloop, 1998). Lead poisoning is known to cause anemia and brain damage, and to affect the central nervous system and kidney functions (Chang, 1988).

Strontium is a naturally occurring alkaline earth metal, but only the radioactive form, ${ }^{90} \mathrm{Sr}$, poses a health risk to humans. Because of chemical similarity to $\mathrm{Ca},{ }^{90} \mathrm{Sr}$ replaces $\mathrm{Ca}$ in the human body and would expose the body to $\beta$-radiation leading to anemia, leukemia, and other chronic illnesses (Chang, 1988). Non-radioactive Cs is also nontoxic. The radioactive isotope of $\mathrm{Cs},{ }^{137} \mathrm{Cs}$, however, is a significant health hazard because it persists for some time in the environment (approximately 300 years) as a highly radioactive element and bioaccumulates through the food chain (Hammond, 1995). Chromium occurs naturally at the earth's surface and is encountered primarily in the + III oxidation state $\left(\mathrm{Cr}^{\mathrm{III}}\right)$, as a metal, in the environment.

Chromium is a natural constituent of living matter and an essential trace element for both plants and animals. At higher concentrations and particularly in the $+\mathrm{VI}$ oxidation state $\left(\mathrm{Cr}^{\mathrm{VI}}\right)$, as the oxyanion chromate $\left(\mathrm{CrO}_{4}{ }^{2-}\right), \mathrm{Cr}$ becomes lethal or can result in severe systemic toxicity and cancer (Squibb and Snow, 1993). Selenium is also known as an essential trace element for a number of organisms. Deficiency of Se is associated with disorders such as ovarian cysts, metritis, and decreased conception rates in cattle. At higher doses, Se is substantially toxic and can cause Se poisoning (Hogan, 1993). Selenium is found in several oxidation states, including $-\mathrm{II}, 0,+\mathrm{IV}$, and $+\mathrm{VI}$. The oxyanion selenite $\left(\mathrm{SeO}_{3}{ }^{2-}\right)$, Se in the $+\mathrm{IV}$ oxidation state, was used in this work. 
Because these contaminants can pose substantial environmental health risks, an understanding of the interaction of each specific ion at mineral-water interfaces is required for any remediation strategy. The experimental results and parameters obtained from this study can reduce the uncertainty of models used to predict the fate and transport of these contaminants at the Frenchman Flat area of the NTS.

\section{MATERIALS AND METHODS}

\section{Materials}

Experiments were conducted with three different volcanic tuff samples from Frenchman Flat on the NTS. All these samples were obtained from well ER5/3/2 and from different depths. Specifically, the three samples were obtained from the following depths below ground surface: 2800, 2960, and $3270 \mathrm{ft}$. Consequently, the three samples were referred to as ER5/3/2 2800D, ER5/3/2 2960D, and ER5/3/2 3270D. The letter D signifies that these samples represent in situ rock, according to the Los Alamos National Laboratory (LANL) researchers who characterized the cuttings. For brevity, these samples are referred to as 2800D, 2960D, and 3270D, respectively.

The cuttings were first ground up (see details below). A fairly extensive characterization of mineral phases followed, including x-ray diffraction (XRD), surface area measurements, micropore area and volume, and average pore diameter determination. The results of quantitative XRD analysis, performed by LANL, and the sample lithology and stratigraphy are shown in Table 1. Different colors were easily observed in the three samples. Sample 2800D had a white color that was thought to result from zeolites, consistent with a high percentage of clinoptilolite and mordenite revealed by XRD analysis. Samples 2960D and 3270D were of orange-red and slightly darker red color, respectively. The red color in these samples was expected to result from iron $(\mathrm{Fe})$ and XRD revealed that hematite and mica concentrations were higher in samples 2960D and 3270D compared to sample 2800D.

Table 1. Physicochemical characteristics of Frenchman Flat samples.

\begin{tabular}{lll}
\hline Core Samples & XRD Results & General Description \\
\hline ER5/3/2-2800D & $\begin{array}{l}\text { feldspar (11.4\%); opal (4.3\%); quartz (2.9\%); } \\
\text { smectite (2.6\%); clinoptilolite (74.2\%); }\end{array}$ & $\begin{array}{l}\text { nonwelded tuff; mafic-poor } \\
\text { Rainier Mesa tuff }\end{array}$ \\
& $\begin{array}{l}\text { mordenite (8.3\%) } \\
\text { cristobalite (4\%); feldspar (71.1\%); }\end{array}$ & $\begin{array}{l}\text { densely welded tuff; crystal- } \\
\text { rich Topopah spring tuff }\end{array}$ \\
& $\begin{array}{l}\text { hematite }(0.5 \%) ; \text { mica (1.9\%); quartz (14\%); } \\
\text { smectite (3.3\%); clinoptilolite (4.2\%) }\end{array}$ & reworked tuff; tuff of \\
ER5/3/2-3270D & $\begin{array}{l}\text { feldspar (63.9\%); hematite (1.1\%); } \\
\text { mica (10.7\%); quartz (18.3\%); smectite (6.4\%) }\end{array}$ & Wahmonie flat \\
\hline
\end{tabular}

Surface area measurement of the different sample size fractions is required for estimation of the sorption capacity of the adsorbents. Because the particle surface area of smooth, nonporous particles is inversely proportional to particle size and the sorption capacity of an adsorbent is directly proportional to the surface area, smaller size fractions are expected to have higher sorption capacity than coarse-sized particles on a per-mass basis. The specific surface area and the pore size distribution of different size fractions, resulting from crushing of the cuttings and sieving, were determined using a Micromeritics ASAP 2010 surface area analyzer and the BET-nitrogen method (Brunauer et al., 1938; Webb and Orr, 1997). This method can be used to estimate the total surface area of adsorbents ranging from a square meter per gram to several hundred square meters per gram. Although the BET method is questionable for surface area 
measurements of microporous materials and swelling clay minerals, it continues to be the most widely used method for particle surface area estimation (Gregg and Sing, 1982). In microporous sorbents in which the average pore diameter is smaller than $20 \AA$, the BET measurement overestimates the total surface area. For swelling clays, such as smectite, the BET method may be underestimating the mineral's specific surface area because nitrogen gas cannot diffuse inside interlayer spaces.

The results of surface area and pore size for different sizes of the Frenchman Flat samples are shown in Table 2. Average pore diameters were measured using the BET method and most pores were considered to be mesopores. Mesopores are defined as pores with average diameter between 20 and $500 \AA$ (Gregg and Sing, 1982). Because the BET isotherm does not provide quantitative information about micropores, micropore volume and area were estimated using the t-plot method, where the statistical thickness of the layer of adsorbed nitrogen is plotted as a function of relative pressure (Gregg and Sing, 1982). Extrapolation of the linear portion to the adsorption axis gives a positive intercept, leading to estimation of micropore volume (Webb and Orr, 1997). The measured micropore volume and area are also shown in Table 2. Because of the relatively low microporosity of the adsorbents and the small fraction of clay minerals (smectite) present, as evidenced by the previously discussed XRD results (Table 1), the measurement of surface area based on the BET method is considered accurate.

Table 2. Specific surface area and pore information for different sized samples.

\begin{tabular}{lcccc}
\hline Core samples & \multicolumn{5}{c}{} \\
\hline ER5/3/2-2800D & $<75 \mu \mathrm{m}$ & $75 \mu \mathrm{m}$ to $125 \mu \mathrm{m}$ & $125 \mu \mathrm{m}$ to $500 \mu \mathrm{m}$ & $2 \mathrm{~mm}$ to $4 \mathrm{~mm}$ \\
Specific surface area $\left(\mathrm{m}^{2} \mathrm{~g}^{-1}\right)$ & 24.4 & 26.0 & 23.1 & 22.8 \\
Average pore diameter by BET $(\AA)$ & 126.2 & 135.0 & 107.3 & 125.7 \\
Micropore volume by t-plot $\left(\mathrm{cm}^{3} \mathrm{~g}^{-1}\right)$ & 0.0037 & 0.0033 & 0.0041 & 0.0027 \\
Micropore area by t-plot $\left(\mathrm{m}^{2} \mathrm{~g}^{-1}\right)$ & 8.32 & 7.57 & 9.10 & 6.18 \\
& & & & \\
ER5/3/2-2960D & & & & \\
Specific surface area $\left(\mathrm{m}^{2} \mathrm{~g}^{-1}\right)$ & 4.51 & 3.40 & 3.15 & 2.58 \\
Average pore diameter by BET $(\AA)$ & 97.8 & 132.0 & 130.4 & 133.1 \\
Micropore volume by t-plot $\left(\mathrm{cm}^{3} \mathrm{~g}^{-1}\right)$ & 0.00069 & 0.00018 & 0.000037 & 0.000021 \\
Micropore area by t-plot $\left(\mathrm{m}^{2} \mathrm{~g}^{-1}\right)$ & 1.53 & 0.52 & 0.05 & 0.03 \\
& & & & \\
ER5/3/2-3270D & & & & \\
Specific surface area $\left(\mathrm{m}^{2} \mathrm{~g}^{-1}\right)$ & 7.94 & 6.61 & 7.29 & 5.91 \\
Average pore diameter by BET $(\AA)$ & 0.0 & 87.6 & 72.9 & 99.2 \\
Micropore volume by t-plot $\left(\mathrm{cm}^{3} \mathrm{~g}^{-1}\right)$ & 0.00069 & 0.00014 & 0.00057 & 0.000017 \\
Micropore area by t-plot $\left(\mathrm{m}^{2} \mathrm{~g}^{-1}\right)$ & 1.61 & 0.47 & 1.35 & 0.13 \\
\hline
\end{tabular}

Because of the presence of smectite and zeolites, these samples showed little dependence of specific surface area on particle size. However, the specific surface area was considerably higher in the 2800D sample compared to the others, suggesting that a higher fraction of porous minerals was present in the 2800D sample. The quantitative XRD study revealed that a high percentage of zeolites (clinoptilolite and mordenite) existed in the 2800D sample. Samples $2960 \mathrm{D}$ and 3270D contained a high fraction of feldspars and quartz that have no internal porosity like zeolites, consistent with the lower surface area estimate. 


\section{Methods}

\section{Batch Sorption/Desorption Equilibrium Experiments}

Particles between 44 and $149 \mu \mathrm{m}$ in diameter, obtained by sieving of the crushed cuttings, were used for batch equilibrium sorption and desorption experiments. Particles were pretreated with $0.1 \mathrm{~N}$ hydrochloric acid $(\mathrm{HCl})$ and $0.1 \mathrm{M}$ sodium nitrate $\left(\mathrm{NaNO}_{3}\right)$ solution to remove the organic coatings present on the surface and easily exchangeable metal ions in the sample. After washing several times with NANOpure ${ }^{\mathrm{TM}}$ water, a leaching process using $0.1 \mathrm{M}$ $\mathrm{NaNO}_{3}$ solution was repeated until the concentration of leached metal in the final solution was negligible. The prewashing of the solid with $\mathrm{NaNO}_{3}$ was necessary to remove any of the intended sorbates that might already be present on the samples. The most problematic of the sorbates is typically $\mathrm{Sr}$ because of the relatively high concentration in many rocks at the NTS. In such cases, without pretreatment, experiments with Sr cannot be conducted.

Synthetic groundwater solutions, including major cations and anions, were prepared based on analysis of groundwater from well U-20 at the NTS (Sloop, 1998). The pH of the volcanic groundwater was 8.3 , and the temperature of the well water was $37^{\circ} \mathrm{C}$. Based on the measured groundwater composition, the ionic strength was calculated as $3.2 \times 10^{-3} \mathrm{M}$ (Sloop, 1998). A variable amount of either $1.0 \mathrm{M}$ nitric acid $\left(\mathrm{HNO}_{3}\right)$ or $0.1 \mathrm{M}$ sodium hydroxide $(\mathrm{NaOH})$ was added to adjust the $\mathrm{pH}$. The amounts of acid and base required for $\mathrm{pH}$ adjustment were determined by trial and error.

All experiments were conducted using reagent-grade chemicals without further purification or modification. High purity water, NANOpure ${ }^{\mathrm{TM}}$ water (at least $18 \mathrm{M} \Omega \mathrm{cm}^{-1}$ resistivity) was provided by a NANOpure water purification system and used for preparing all solutions. Lead $(\mathrm{Pb})$, strontium $(\mathrm{Sr})$, cesium $(\mathrm{Cs})$, chromate $(\mathrm{Cr})$, and selenite $(\mathrm{Se})$ were prepared as lead nitrate $\left(\mathrm{Pb}\left(\mathrm{NO}_{3}\right)_{2}\right)$, strontium nitrate $\left(\mathrm{Sr}\left(\mathrm{NO}_{3}\right)_{2}\right)$, cesium nitrate $\left(\mathrm{CsNO}_{3}\right)$, potassium chromate $\left(\mathrm{K}_{2} \mathrm{CrO}_{4}\right)$, and sodium selenite $\left(\mathrm{Na}_{2} \mathrm{SeO}_{3}\right)$ solutions, respectively. In the rest of the report, the oxyanions of $\mathrm{Cr}^{\mathrm{VI}}$ and $\mathrm{Se}^{\mathrm{IV}}$, chromate and selenite, respectively, will be represented by $\mathrm{Cr}$ and Se. It should be emphasized, however, that the oxyanions of these elements, in the respective oxidation states, are implied

A set of eight to ten test tubes was prepared at the same time for the $\mathrm{pH}$-dependent experiments. The metal solution of interest was introduced in the individual test tube and mixed with weighed quantities of the crushed particles. Metal cation sorption experiments were conducted with $3 \mathrm{~g} / \mathrm{L}$ of solid concentration and $10^{-5} \mathrm{M}$ of initial metal concentration. Sorption experiments for anions were performed with $10 \mathrm{~g} / \mathrm{L}$ solid concentration and $10^{-6} \mathrm{M}$ metal concentration.

The individual test tubes were equilibrated for 48 hours by end-over-end rotation at 8 $\mathrm{rpm}$. End-over-end rotation was used to accelerate equilibration times. Comparison of preliminary experimental tests using different equilibration times of 1, 2, 3, and 7 days showed no significant differences in these data sets. Following equilibrium, the $\mathrm{pH}$ of the suspension was measured using an Orion model $720 \mathrm{pH}$ meter with an Orion Ross glass combination electrode. Solid-solution separation was achieved using a Marathon K/R 21 centrifuge by centrifugation at 9,000 rpm for 30 minutes and a $1-\mathrm{mL}$ aliquot of the supernatant was removed for metal analysis. The supernatant was acidified with $12 \mu \mathrm{L}$ of concentrated $\mathrm{HNO}_{3}$ (Fisher OptimaTM quality) before analysis. 
The metal concentration was determined using a Perkin Elmer 4110 ZL atomic absorption (AA) spectrometer with graphite furnace and Zeeman background correction. The fractional sorption uptake of the ions of interest was determined by the difference in concentrations in the supernatant to the concentration of a blank sample, in which no solid had been added. Duplicate analyses were run for each sample and the results were averaged. Individual samples were prepared under the same conditions to determine the variation of final sorption uptake and $\mathrm{pH}$. Duplicate samples revealed no significant variations among samples.

Desorption experiments were conducted following the sorption experiments. Usually eight to ten test tubes were prepared under the same conditions and individual test tubes were analyzed as a function of time. The $\mathrm{pH}$ was maintained constant throughout the experiment around 8.0. After sorption equilibrium was reached, the supernatant was centrifuged and decanted carefully. The metal concentration in the solution was measured by AA and the sorbed metal concentration on the solid was determined by the difference between blank concentration and metal concentration in the supernatant after centrifugation. The desorption solution was essentially the background electrolyte solution. Test tubes were mixed again by end-over-end rotation for a predetermined equilibration time. Each test tube was centrifuged after the predetermined equilibration time and the metal concentration desorbed from the solid was measured in the supernatant. The same synthetic groundwater solutions used for the previous sorption experiments were prepared to test the reversibility of sorption for each metal ion on the three different Frenchman Flat samples.

Sorption/Desorption Isotherm Derivation

Sorption and desorption isotherms of ions on Frenchman Flat volcanic tuffs under different geochemical conditions were obtained by changing metal and solid concentrations. For the desorption isotherms, sample analysis was conducted following a 30-day equilibration time period, a period long enough to ensure that equilibrium was reached. Sorption isotherms have been used to predict the sorption and migration of contaminants in the subsurface environment. The most commonly used isotherms are the linear, Freundlich, and Langmuir. Even though the linear isotherm of inorganic contaminants is dependent on $\mathrm{pH}$, possibly ionic strength, and solution composition, use of the $K_{\mathrm{d}}$ to model contaminant partitioning at mineral-water interfaces has been widely used, because of its simplicity and straightforward incorporation in transport models. The $K_{\mathrm{d}}$ for sorption and desorption isotherms were determined by Equation (1)

$$
K_{\mathrm{d}}=\frac{S}{C_{e q}}
$$

where $S$ is the concentration of sorbed ions (sorbed metal ion mass per mass of sorbent, $\mathrm{M} \mathrm{M}^{-1}$ ) and $C_{e q}$ is the equilibrium metal ion concentration in solution $\left(\mathrm{M} \mathrm{L}^{-3}\right)$.

\section{Rate of Uptake Experiments}

The experiments to determine the rates of metal ion uptake were conducted using spherical coarse particles with an average diameter of $2.2 \mathrm{~mm}$. The particles were equilibrated with NANOpure $^{\mathrm{TM}}$ water in 250-mL Nalgene centrifuge tubes for at least 24 hours. An additional 24-hour saturation was conducted in synthetic groundwater solution at constant $\mathrm{pH}$ around 8.0. After equilibrium of the particles at the desired $\mathrm{pH}$, the metal ion was introduced into the centrifuge tube and mixed by shaker. Negligible particle loss or abrasion was found during the experiment. Metal and solid concentrations for the rate of uptake experiments were similar to 
the concentrations used in the sorption experiments, typically $10^{-5} \mathrm{M}$ metal and $3 \mathrm{~g} / \mathrm{L}$ solid. The addition of the metal ion solution to the centrifuge tube marked the beginning of the experiment. Samples were withdrawn as a function of time with a $10-\mathrm{mL}$ plastic syringe equipped with a three-way stopcock and a $0.02-\mu \mathrm{m}$ nylon filter. A sample of only $0.2 \mathrm{~mL}$ was withdrawn to minimize the change of solid to liquid ratio and the metal concentration was determined by AA. Because the rate of uptake was fast early on during the experiment, more frequent sampling was conducted during the early stages of the experiment. The $\mathrm{pH}$ of the solution was also measured at the same time as sample collection and the $\mathrm{pH}$ variation during the experiment was within 0.1 units.

\section{X-ray Photoelectron Spectroscopy (XPS)}

Background. This section is intended as a brief introduction to x-ray photoelectron spectroscopy (XPS). A thorough introduction to this important technique is, obviously, beyond the scope of this report. The interested reader should consult the additional references given below. A very brief introduction to XPS is given by Hochella (1990). A concise overview of the technique, its history, the basic theory behind it, and some applications are given by Kelly (1986). Briggs and Seah (1990) give a complete coverage of the technique including instrumentation, spectral interpretation, quantification, and an extended discussion of various technological applications. A fairly basic introduction to XPS with emphasis on spectral interpretation and accompanied by a discussion of applications in geology is given by Perry et al. (1990). Hochella (1988) gives a more complete overview of the technique including basic theory, spectral interpretation, instrumentation, sample preparation, and applications in geology, mineralogy, and geochemistry. Finally, a substantially more theoretical treatment of the subject is presented by Brundle and Baker (1978).

Basic Theory. X-ray photoelectron spectroscopy is based on the photoelectric effect. When materials are illuminated with X-rays energetic enough to eject electrons from inner- or valence shells, the difference between the incoming photon energy and electron binding energy (BE) is converted to kinetic energy of the escaping photoelectron. Binding energies for different elements and orbitals can be determined either experimentally or from single-atom models. XPS is a surface sensitive technique because it is based on detection of photoelectrons, which have, essentially, undergone no inelastic collisions. This can only happen if the atoms from which electrons are ejected are located near the surface. The depth of analysis is therefore typically between 10 and $50 \AA$. Flat or polished surfaces, as well as rocks and powders can be analyzed, although certain types of studies require flat surfaces. The typical area of analysis depends on the particular instrument and can vary from several millimeters down to a few micrometers. The SSX-100 instrument used in this study is capable of analysis using a spot size between 100 and $800 \mu \mathrm{m}$.

Spectral Interpretation and Chemical Shifts. Because every element has a unique atomic structure and the low energy $x$-rays used can only excite a few atomic levels from each element, elements can be identified unambiguously in most cases. A wide-energy-range "survey" scan can be used to identify the elements present in the sample but it is not used for quantification of elemental composition because it lacks resolution; narrow scans are used for this purpose. Contaminants in the air and residual gases in the vacuum chamber give rise to a C 1s peak, referred to as the "adventitious carbon" peak.

A chemical shift is defined as the difference in BE between a particular line and the BE for the same line in a reference compound. The chemical shift is a function of the environment of 
an atom. By means of chemical shifts, one can often distinguish between two different oxidation states of an element in a given sample or between different coordination environments.

Charge Referencing. When insulators are being analyzed, the sample is necessarily insulated from the spectrometer and the surface is charged positively as photoelectrons are ejected from the sample. This surface charging results in observed BE shifts typically between 5 and $10 \mathrm{eV}$. To obtain accurate BEs for insulators, the magnitude of charge shifting must be estimated. This procedure is called charge referencing and is usually accomplished by the adventitious carbon, the gold dot, or the argon implantation method. The adventitious carbon method uses the presence of adventitious carbon on practically every sample and assigns a $\mathrm{BE}$ to the $\mathrm{C} 1 \mathrm{~s}$ line. Because reported values for the adventitious $\mathrm{C} 1 \mathrm{~s}$ line range from 284.6 to $285.2 \mathrm{eV}$, the values determined with this method may be off by as much as $0.6 \mathrm{eV}$.

Chemical Quantification. With XPS, one can obtain at least semi-quantitative estimates of the relative abundance of the elements present on the surface of a sample. To obtain reliable quantitative information, narrow scans of the regions corresponding to the peaks of interest must be collected using small energy steps. Quantification, however, from wide (survey) scans has also been attempted (Turner, 1992). If only the ratio of two elements is needed, as is often the case in surface analysis, the relative abundance of the two elements can be estimated from the photoionization cross sections and the intensities of the two peaks. The photoionization cross section gives the probability that a photon will be absorbed by an electron in a specific orbital. Theoretical photoionization cross sections were tabulated by Scofield (1976). Quantification, therefore, only requires measurement of the intensities of the two peaks and knowledge of the corresponding cross sections, as well as knowledge of the sensitivity of the analyzer for the specific electron energy involved. An alternative approach, however, which may give better results, is to use empirical cross sections derived from well-defined crystalline materials, where the elements whose ratio must be determined in the unknown are present.

\section{RESULTS AND DISCUSSION}

\section{Sorption Experiments}

It is well known that the fractional uptake of cations by oxide and hydroxide minerals is highly pH dependent (Davis and Leckie, 1980; Stumm et al., 1980; Hayes and Leckie, 1987; Katz and Hayes, 1995). This is because sorption of cations and anions on oxides and hydroxides is controlled by sorption on $\mathrm{pH}$-dependent, surface hydroxyl sites that can be protonated at low $\mathrm{pH}$ and deprotonated at high $\mathrm{pH}$. At low $\mathrm{pH}$, adsorption of cations is minimal, because the mineral surfaces have a positive charge resulting from protonation (Drever, 1997). As the $\mathrm{pH}$ increases, surface charge becomes more negative and sorption uptake increases as well. Different oxides and hydroxides display different $\mathrm{pH}$-dependent behavior, depending on the acidity of the surface hydroxyl sites, similar to acid-based systems that are characterized by their $\mathrm{pK}_{\mathrm{a}} \mathrm{s}$. Cation sorption on clay minerals may or may not be $\mathrm{pH}$ dependent. Sorption on non-swelling 1:1 clays (e.g., kaolinite) with primarily surface-hydroxyl sorption sites is expected to be highly $\mathrm{pH}$ dependent (O'Day et al., 1994a, b), whereas sorption on smectites (2:1 expanding clays) may or may not be $\mathrm{pH}$ dependent, depending on other geochemical conditions (Papelis and Hayes, 1996).

The sorption uptake of metal cations such as $\mathrm{Sr}, \mathrm{Cs}$, and $\mathrm{Pb}$, and the anions $\mathrm{Cr}$ and $\mathrm{Se}$ onto three different Frenchman Flat (FF) volcanic tuff samples was evaluated in a synthetic groundwater solution as a function of $\mathrm{pH}$. The sorption uptake of $\mathrm{Sr}$ by the three samples is shown in Figure 1. Because the same synthetic groundwater solution was used for all Sr sorption 
experiments, the ionic strength was the same during all experiments, approximately $0.003 \mathrm{M}$. The $\mathrm{pH}$ and mineral composition of the substrates were the only different parameters resulting in different sorption results.

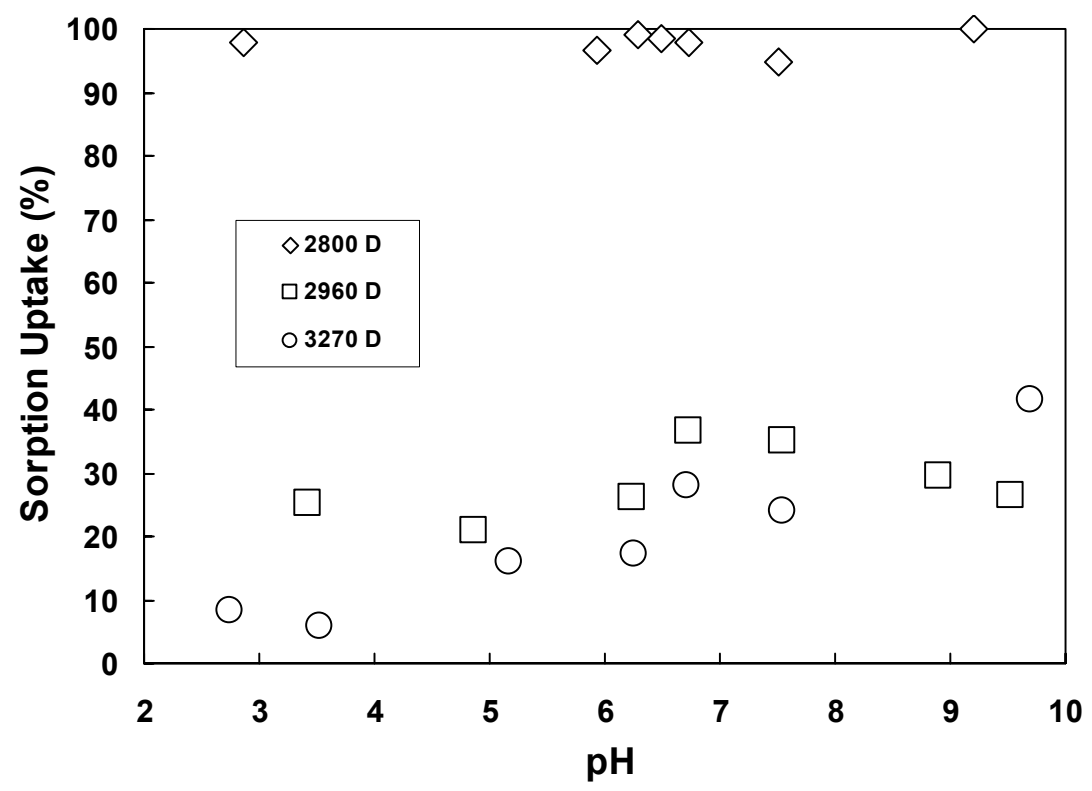

Figure 1. Sorption of $10^{-5} \mathrm{M} \mathrm{Sr}$ on $3 \mathrm{~g} / \mathrm{L}$ of samples 2800D, 2960D, and 3270D.

The results of $\mathrm{Sr}$ sorption were noticeably different for the three samples. Sample 2800D resulted in essentially quantitative (over 95\%) removal of $\mathrm{Sr}$ from solution, regardless of $\mathrm{pH}$. Because of the relatively high percentage of zeolites in the sample (clinoptilolite and mordentite), sample 2800D had higher surface area and therefore showed higher Sr sorption uptake compared to the other samples. Zeolites are hydrated aluminosilicate minerals and because of their molecular sieve structure and high CEC, zeolites have been used to remove radionuclides and metal cations (Ames, 1960; Faghihian et al., 1999). A white-colored zeolitized tuff collected from Rainier Mesa, which is similar to sample 2800D, had high sorption capacity for $\mathrm{Sr}$ and $\mathrm{Pb}$ at ionic strengths higher than used here $\left(\mathrm{I}=0.01 \mathrm{M} \mathrm{NaNO}_{3}\right)$, regardless of $\mathrm{pH}$ (Sloop, 1998; Um, 2001).

Because of the high CEC in zeolites, most Sr ions presumably sorb onto permanently negatively charged ion-exchange sorption sites, located inside channels of zeolites, resulting in $\mathrm{pH}$-independent sorption, rather than on surface hydroxyl sites at mineral edges, where surface charge is expected to be $\mathrm{pH}$ dependent. Sorption of $\mathrm{Sr}$ on 2960D was reduced (average 30\%) and Sr sorption appeared to be only slightly $\mathrm{pH}$ dependent, with similar fractional uptake at both low and high $\mathrm{pH}$. The overall reduced sorption uptake can be explained by the substantially reduced zeolite content of this sample, compared to $2800 \mathrm{D}$. In this sample, however, the majority of sorption sites were expected to be $\mathrm{pH}$-independent, cation exchange sites associated with mica, smectite, and clinoptilolite. Sorption on sample 3270D suggested a different sorption behavior, namely a slightly $\mathrm{pH}$-dependent sorption uptake, with fractional $\mathrm{Sr}$ uptake increasing from approximately 10 percent at low $\mathrm{pH}$ to essentially 40 percent at high $\mathrm{pH}$. Because the $3270 \mathrm{D}$ sample did not contain any zeolites, ion-exchange sites might only come from smectite or mica. Based on the observed behavior, one must conclude that the relative abundance of amphoteric 
surface hydroxyl sites compared to permanent charge cation exchange sites was higher in sample 3270D compared to the other samples.

Results of Cs uptake were qualitatively similar to the Sr results. Cesium sorption results are shown in Figure 2. Essentially complete (100\%) sorption uptake of Cs was found in sample 2800D. As described before, because of the high fraction of zeolites in this sample, Cs sorption uptake was the highest in 2800D. The fractional uptake of Cs by $2960 \mathrm{D}$ was approximately 60 to 70 percent and essentially $\mathrm{pH}$ independent, except at very low $\mathrm{pH}$, approximately 2 to 3 .

Compared to $\mathrm{Sr}, \mathrm{Cs}$ appeared to bind stronger on 2960D. This observation is consistent with results previously reported in the literature (Rajec et al., 1998). Based on these experiments, Cs sorption on zeolites and smectite minerals appeared to be the predominant mechanism of sorption on 2960D. The slight increase in sorption uptake with increasing $\mathrm{pH}$ could be attributed to sorption on surface hydroxyl sites on aluminosilicate minerals. Obviously, the majority of sorption sites appears to be associated with permanent charge cation exchange sites on zeolites and smectite clay minerals rather than surface hydroxyl sites on quartz and feldspars, or edge sites of smectite. The rather dramatic decrease in sorption at $\mathrm{pH}$ below 3 could be attributed to competition for cation exchange sites with the hydrogen ion. At these low $\mathrm{pH}$ values, the concentration of the hydrogen ion becomes significant and could compete successfully with the Cs cation for cation exchange sites.

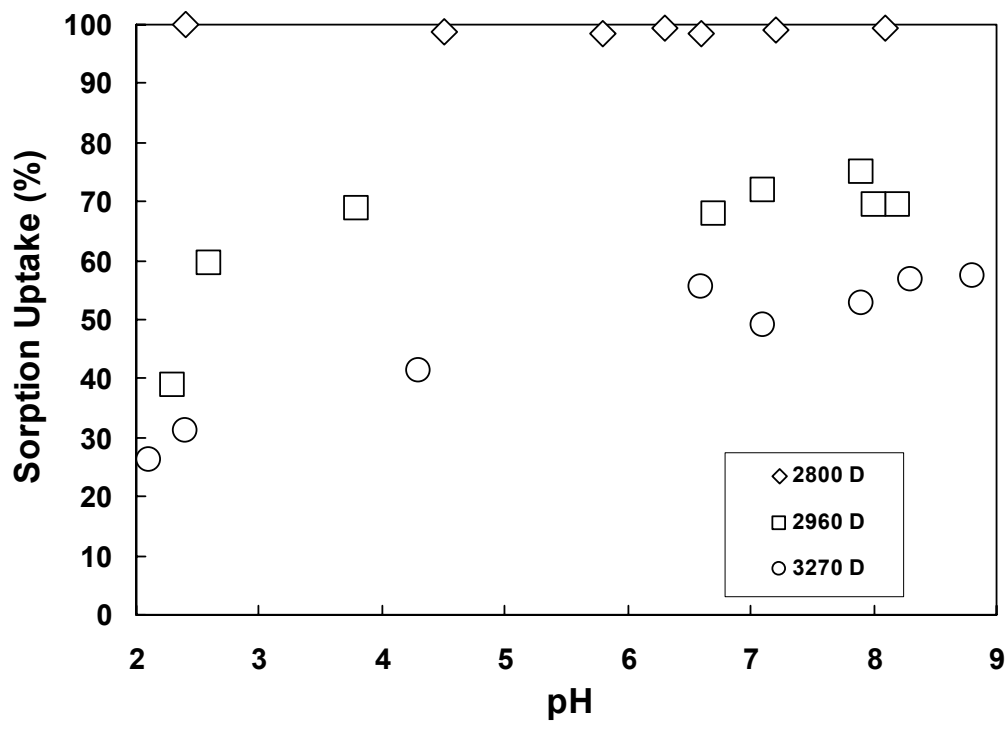

Figure 2. Sorption of $10^{-5} \mathrm{M} \mathrm{Cs}$ on $3 \mathrm{~g} / \mathrm{L}$ of samples 2800D, 2960D, and 3270D.

Sorption of Cs on 3270D was both quantitatively and qualitatively different compared to the other two samples. Quantitatively, Cs sorption on 3270D was reduced compared to sorption on 2800D and 2960D. In addition, while Cs sorption on 2800D was $\mathrm{pH}$ independent and sorption on 2960 was essentially $\mathrm{pH}$ independent, except at very low $\mathrm{pH}$, sorption on 3270D was considerably more $\mathrm{pH}$ dependent. Specifically, Cs fractional uptake increased from approximately 25 percent at $\mathrm{pH} 2$ to 50 to 60 percent at $\mathrm{pH} 9$. Assuming that the $\mathrm{pH}$-independent fraction can be attributed to sorption on cation exchange sites and the $\mathrm{pH}$-dependent fraction can be attributed to sorption on surface hydroxyl sites, it appears that at least 50 percent of Cs sorption on 3270D could be attributed to sorption on surface hydroxyl sites. This hypothesis appears to be consistent with the mineralogical characterization of this sample. According to the 
quantitative XRD analysis, 3270D contained no zeolites and instead it contained the highest fraction of hematite and quartz (Table 1). Only smectite clays could be responsible for sorption on cation exchange sites on this sample. Based on the results shown in Figure 2, approximately half of the observed sorption could be attributed to sorption on cation exchange sites, given that the sorption at the lowest $\mathrm{pH}$ values (less than 3 ) is unlikely to be attributed to sorption on surface hydroxyl sites.

The sorption behavior of $\mathrm{Pb}$ was different from $\mathrm{Sr}$ and $\mathrm{Cs}$ (Figure 3). Because of the high selectivity of $\mathrm{Pb}$ for cation exchange sites (Faghihian et al., 1999; Inglezakis et al., 2002; Langella et al., 2000), Pb sorption uptake by both 2800D and 2960D was essentially complete, over 90 to 95 percent. The slightly reduced $\mathrm{Pb}$ sorption uptake by 2960D at low pH probably resulted from competition with hydrogen ions for cation exchange sites. The $\mathrm{Pb}$ sorption results on 3270D were consistent with the hypothesis regarding Cs sorption on 3270D outlined above. Although the $\mathrm{pH}$ dependence of $\mathrm{Pb}$ sorption was much more pronounced compared to $\mathrm{Cs}$, the main conclusions are the same. A fraction of the total sorption can be attributed to sorption on cation exchange sites and, by comparing Figures 2 and 3, this fraction is estimated to be approximately 20 to 30 percent, while the rest of sorption must be attributed to sorption on surface hydroxyl sites. Because the affinity of $\mathrm{Pb}$ for surface hydroxyl sites is much stronger compared to $\mathrm{Cs}$, the $\mathrm{pH}$ dependent part of $\mathrm{Pb}$ sorption was much more pronounced and resulted in almost complete $\mathrm{Pb}$ removal around neutral $\mathrm{pH}$ and above.

Based on the sorption results with the three different metal cations, $\mathrm{Pb}$ had the strongest sorption affinity for all samples. Cesium and Sr appeared to bind significantly less, under most conditions. This is expected, unless cation exchange sites, because of high zeolite content of the

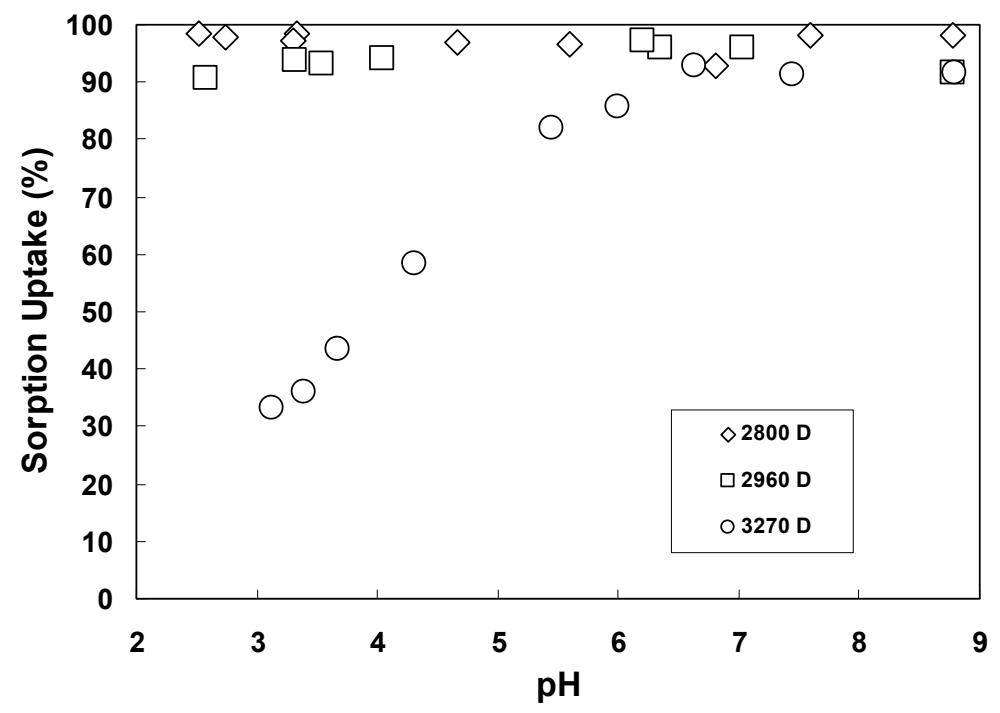

Figure 3. Sorption of $10^{-5} \mathrm{M} \mathrm{Pb}$ on $3 \mathrm{~g} / \mathrm{L}$ of samples 2800D, 2960D, and 3270D.

sample, are in substantial excess, compared to the metal ion concentration. The fact that at least 95 percent of all three cations was removed by sorption onto sample 2800D, the sample with the highest concentration of zeolites, points to the high efficiency of these sorbents in removing radionuclides and other cationic contaminants from solution. 
The sorption of anions and oxyanions on smectites and zeolites, the primary sources of cation exchange sites in a system, is minimal resulting in substantially lower retardation of anions compared to cations. Because of the potential adverse health effects of the oxyanions of technetium and chromium, sorption experiments with anions were also conducted to estimate if these anions would be retarded in the subsurface environments by interactions with Frenchman Flat samples. To better bracket the expected behavior of anions, experiments were conducted with a strongly binding anion, selenite $\mathrm{Se}^{\mathrm{IV}}$, and with a weakly binding anion, chromate $\mathrm{Cr}^{\mathrm{VI}}$.

Higher solid concentration and lower total initial concentration were used for the anion sorption experiments compared to the cation sorption experiments to achieve any measurable fractional sorption uptake. The Se sorption uptake by the three different samples is given in Figure 4. Selenite sorption was $\mathrm{pH}$ dependent, highest at low $\mathrm{pH}$ and decreasing with increasing $\mathrm{pH}$. Because of protonation-deprotonation reactions on amphoteric surface hydroxyl sites of oxides and hydroxides, sorption of anions is known to be $\mathrm{pH}$ dependent, increasing with decreasing $\mathrm{pH}$, as has been established by previous studies (Hingston et al., 1972, Stumm et al., 1980, Davis and Leckie, 1980).

The highest Se uptake was observed on sample 3270D at low $\mathrm{pH}$. In addition, uptake of Se by $2800 \mathrm{D}$ above neutral $\mathrm{pH}$ was negligible. These results are expected because of the higher content of oxide minerals in sample 2960D and especially 3270D, compared to sample 2800. Because zeolites possess a net negative structural charge, resulting from isomorphic substitution of cations in the structural lattice, they tend to have little or no affinity for anionic ions (Haggerty and Bowman, 1994). The same is true for smectite clay minerals. The high content of zeolites and small fraction of oxide minerals in $2800 \mathrm{D}$ resulted in relatively small Se sorption uptake compared to the other two samples.

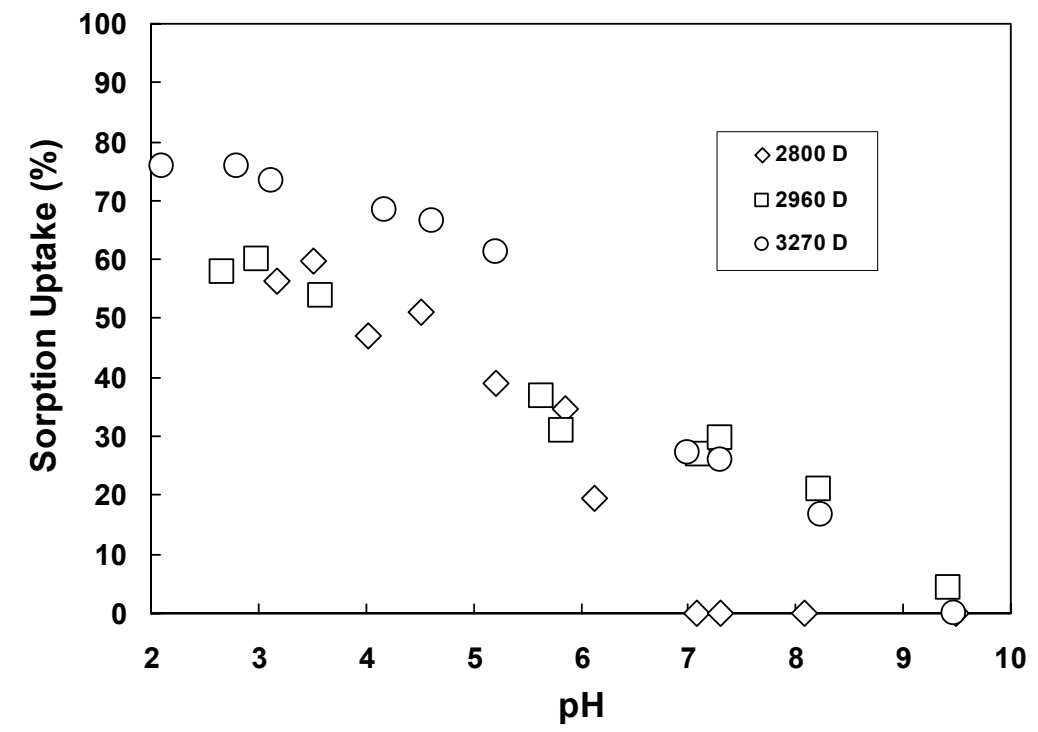

Figure 4. Sorption of $10^{-6} \mathrm{M}$ Se on $10 \mathrm{~g} / \mathrm{L}$ of samples 2800D, 2960D, and 3270D.

Chromate sorption on all samples is shown in Figure 5. Chromate sorption uptake was negligible on both 2800D and 2960D samples over the entire $\mathrm{pH}$ range. Small fractional $\mathrm{Cr}$ removal was only found on sample 3270D at very low $\mathrm{pH}$, consistent with the higher content of oxide minerals in 3270D compared to the other samples. Compared to Se sorption results, $\mathrm{Cr}$ 
was assumed to be a weakly binding anion. Selenite is considered a strongly binding anion on hydrous oxide surfaces and the formation of inner-sphere surface complexes on transition aluminas and goethite was verified by spectroscopic techniques (Hayes et al., 1988; Papelis et al., 1995). However, although high Se sorption uptake was found at low $\mathrm{pH}$, because of small Se sorption affinity at high $\mathrm{pH}$, the migration of Se would be enhanced compared to cations under natural conditions at the NTS $(\mathrm{pH}=8.3)$. Because of the abundance of $\mathrm{Cr}$ in the subsurface environment at the NTS and negligible $\mathrm{Cr}$ sorption on solids above neutral $\mathrm{pH}, \mathrm{Cr}$ would not be retarded significantly with respect to groundwater flow due to sorption on Frenchman Flat aquifer materials.

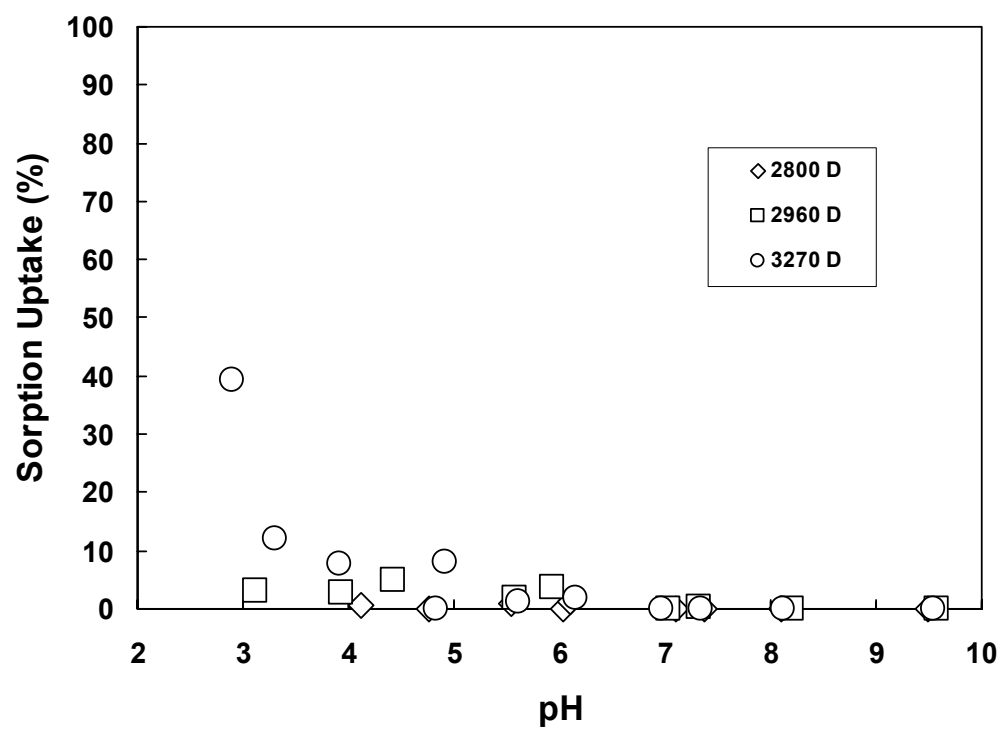

Figure 5. Sorption of $10^{-6} \mathrm{M} \mathrm{Cr}$ on $10 \mathrm{~g} / \mathrm{L}$ of samples $2800 \mathrm{D}, 2960 \mathrm{D}$, and 3270D.

\section{Desorption Experiments}

The desorption behavior of radionuclides and other contaminants is also required to estimate the mobility and the sorption reversibility of contaminants in an aqueous environment. In considering the reversibility of sorption, if the sorption uptake of a contaminant is reversible, although the initial sorption may be high, the initially sorbed ions may be released from the solid phase into the aqueous phase, thereby enabling the migration of contaminants. The objective of this experiment was to investigate the sorption reversibility of $\mathrm{Sr}, \mathrm{Cs}$, and $\mathrm{Pb}$ on Frenchman Flat aquifer materials. Because anions such as Se and $\mathrm{Cr}$ revealed negligible sorption uptake at the $\mathrm{pH}$ of NTS groundwater, desorption experiments with these anions were not conducted.

The results of $\mathrm{Sr}$ desorption are shown in Figure 6. The desorption release of $\mathrm{Sr}$ from $\mathrm{Sr}$ initially sorbed on 2800D was negligible, suggesting strong Sr sorption on this sample, consistent with the sorption experiments. However, up to an average of 80 percent of Sr initially sorbed on samples 2960D and 3270D was released during the desorption step. The reduced amounts of desorption after 30 days were attributed to limited re-adsorption onto the adsorbents. The relatively high desorption release from samples 2960D and 3270D is indicative of the relatively weak binding of $\mathrm{Sr}$ onto these solids. As discussed in the previous section, sorption on amphoteric surface hydroxyl sites was more important for samples 2960D and 3270D than 2800D. Spectroscopic investigations have always shown that $\mathrm{Sr}$ binds by formation of outer- 
sphere complexes, regardless of geochemical conditions (Chen et al., 1998; Um, 2001). The formation of outer-sphere complexes on surface hydroxyl sites has been correlated with increased ionic-strength dependence of sorption (Hayes et al., 1987). Strontium bound on amphoteric surface hydroxyl sites of samples 2960D and 3270D would be expected to desorb more readily compared to $\mathrm{Sr}$ bound by cation exchange in zeolites of sample 2800D.

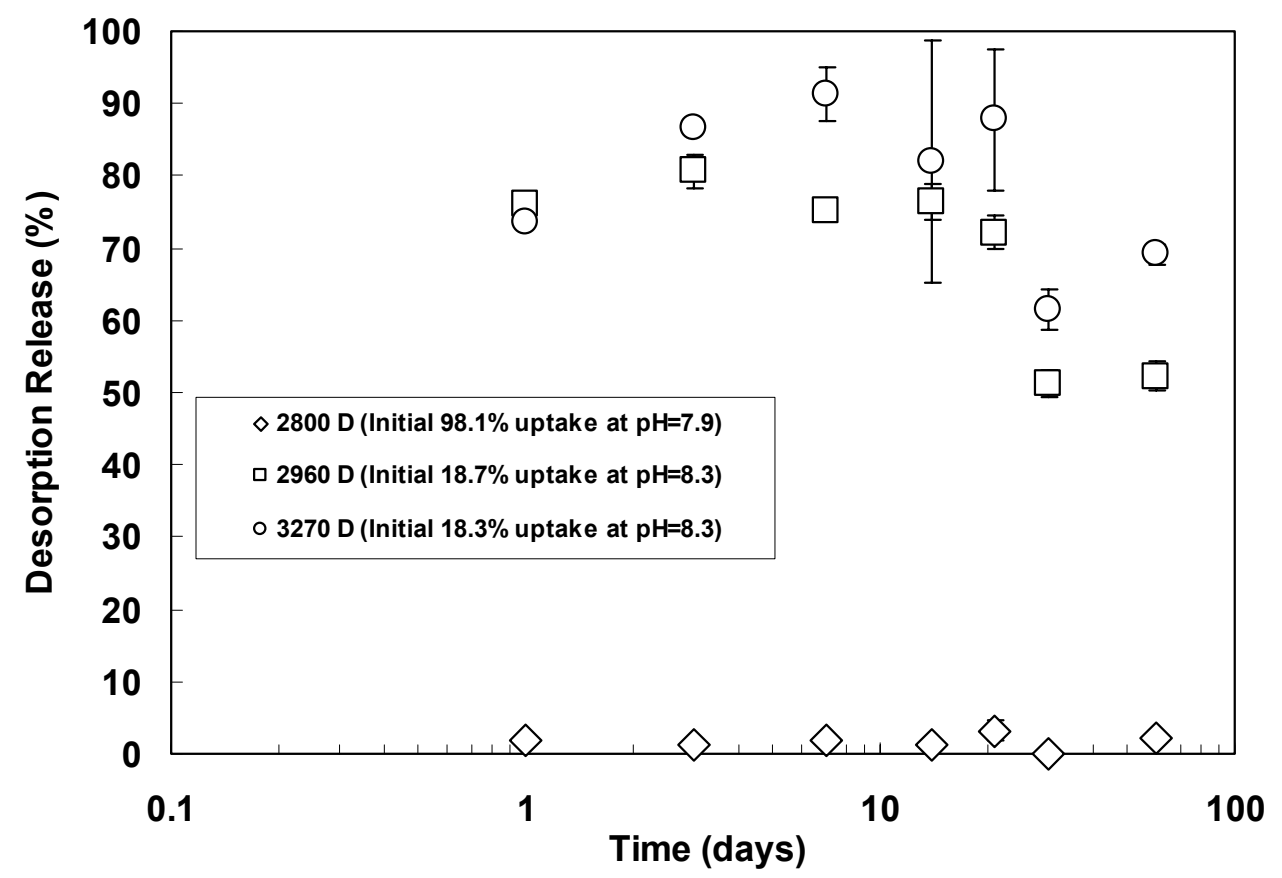

Figure 6. Percent Sr release following sorption of $10^{-5} \mathrm{M}$ Sr on $3 \mathrm{~g} / \mathrm{L}$ of samples 2800D, 2960D, and 3270D.

The desorption of Cs is shown in Figure 7. Qualitatively, the Cs desorption behavior was similar to Sr. The smaller amounts of Cs desorbed, however, indicate relatively stronger sorption affinity of Cs for samples 2960D and 3270D compared to Sr, under these conditions. Sample 2800D had strong sorption capacity for Cs as well, based on the fact that essentially no Cs was released from 2800D even after 60 days. The fractional release of Cs from samples 2960D and $3270 \mathrm{D}$ after one day averaged 20 percent and 30 percent, respectively. These results, again point to the differences in sorption mechanisms responsible for cation sorption on these three samples. Sorption on sample 2800D appears to be controlled entirely by sorption on cation exchange sites, whereas sorption on 2960D and 3270D involves, at least partially, sorption on surface hydroxyl sites through outer-sphere complex formation. Sorption of Sr by cation exchange appears to be irreversible under the conditions tested, whereas formation of outer-sphere complexes appears to be partly reversible. 


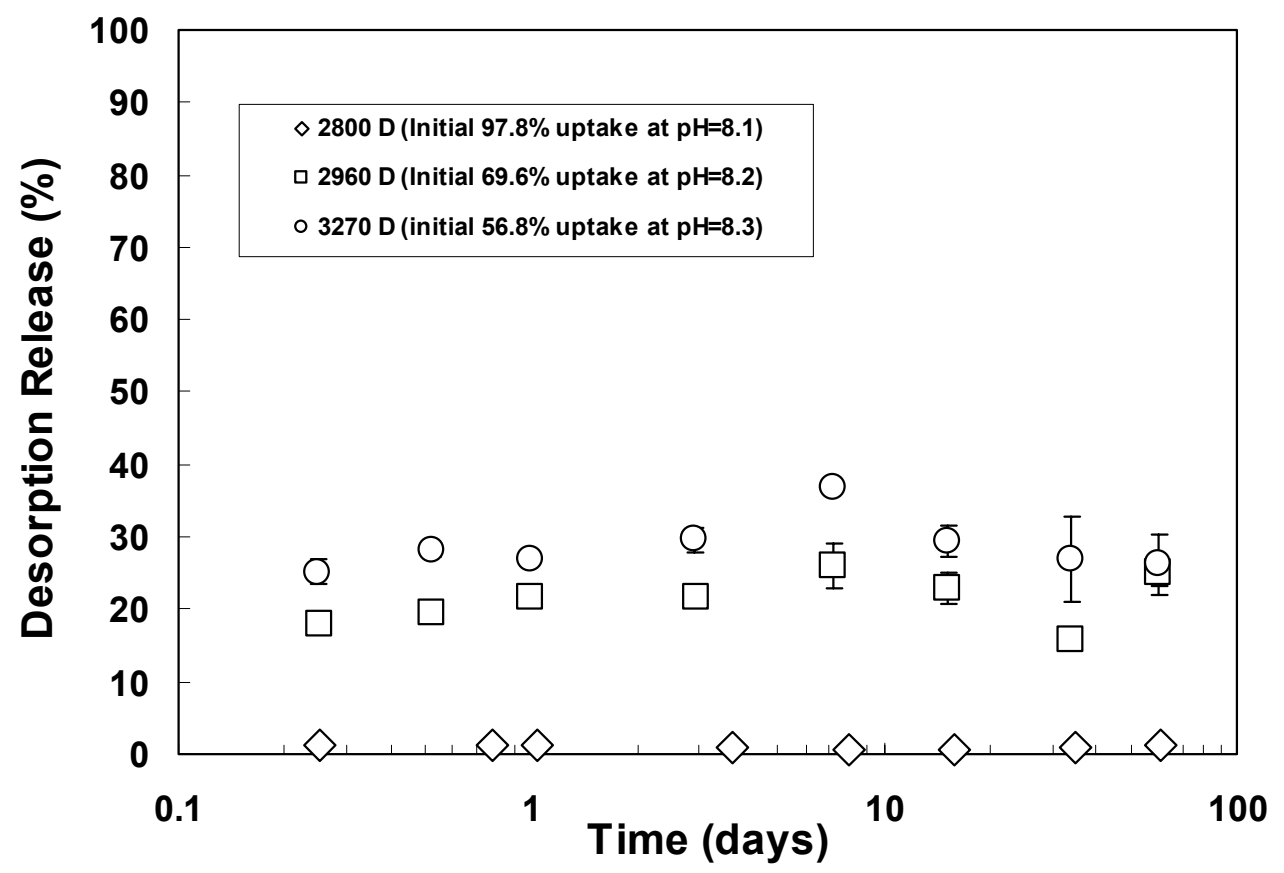

Figure 7. Percent Cs release following sorption of $10^{-5} \mathrm{M} \mathrm{Cs}$ on $3 \mathrm{~g} / \mathrm{L}$ of samples $2800 \mathrm{D}, 2960 \mathrm{D}$, and 3270D.

Lead desorption release from the three samples indicated much stronger sorption compared to $\mathrm{Sr}$ and $\mathrm{Cs}$ (Figure 8). Although the sorption experiments were conducted under the same conditions, only $\mathrm{Pb}$ ions were marginally released from all Frenchman Flat samples, almost independently of sample mineralogy. The strong affinity of $\mathrm{Pb}$ for these surfaces can be explained based on the following considerations. The affinity of a cation for ion-exchange sites is a function of the charge and size of the cation (Bohn et al., 1985; McBride, 1994). Lower charge and bigger crystallographic radius of ion results in a smaller radius of hydrated ion allowing closer proximity to the charged surface, thereby leading to stronger sorption complexes.

Both $\mathrm{Pb}$ and $\mathrm{Sr}$ are divalent cations, so that charge does not affect their relative affinity for ion-exchange sites. The crystallographic radii of these two cations, however, differ, the average reported radius of $\mathrm{Pb}^{\mathrm{II}}$ being $1.25 \AA$ and the average reported radius of $\mathrm{Sr}^{\mathrm{II}}$ being $1.20 \AA$ (Jaffe, 1988). Since the hydrated radius of an ion is inversely proportional to its crystallographic (dehydrated) radius (Bohn et al., 1985), the hydrated radius of $\mathrm{Pb}$ was expected to be smaller than the hydrated radius of $\mathrm{Sr}$, consistent with the observed stronger sorption affinity of $\mathrm{Pb}$ for ion-exchange sites compared to Sr. And although a difference of $0.05 \AA$ may not seem important, the hydrolysis behavior of the two ions is dramatically different (Baes and Mesmer, 1986), resulting in significantly different coordination numbers in aqueous solutions, namely approximately 8 for $\mathrm{Sr}$ and 2 to 3 for $\mathrm{Pb}$ (Um and Papelis, submitted). 


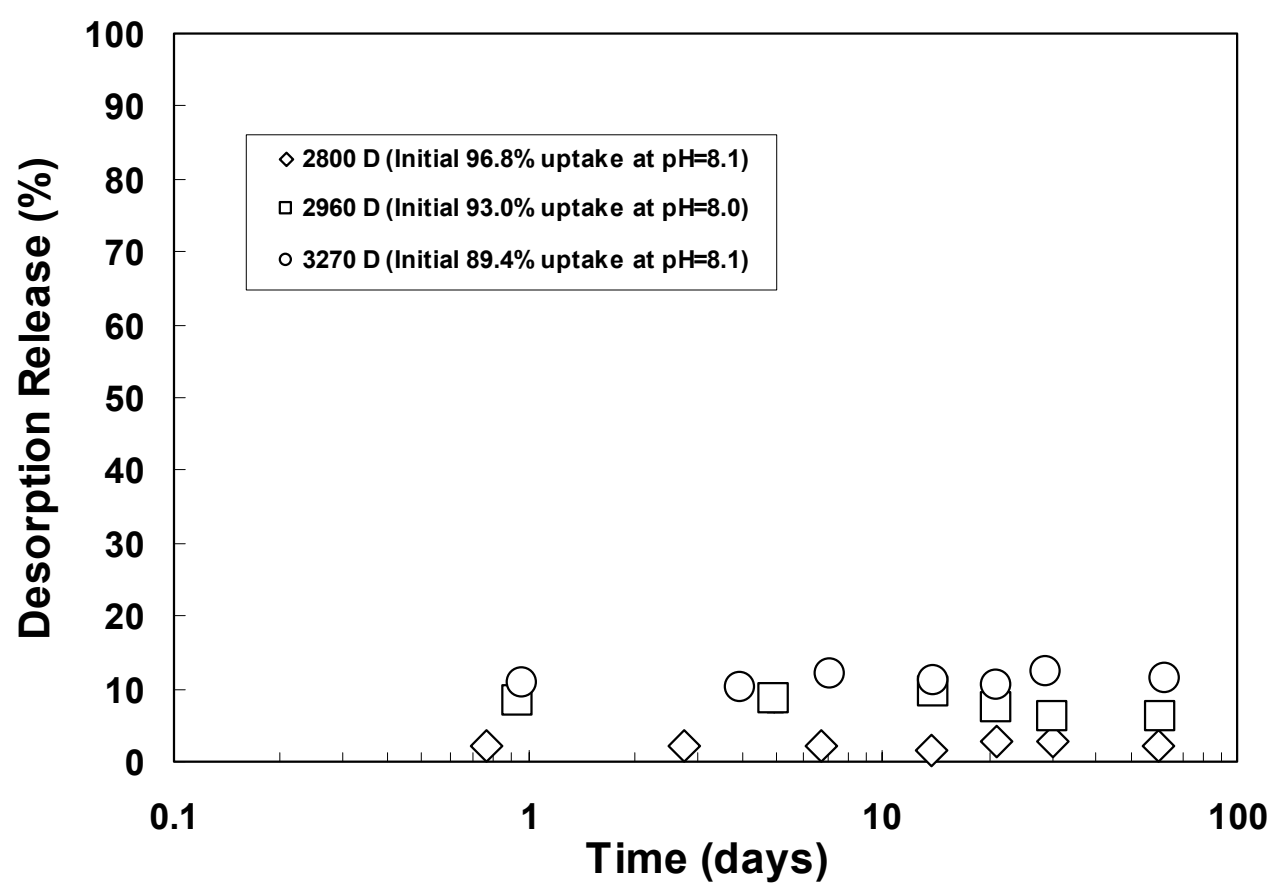

Figure 8. Percent $\mathrm{Pb}$ release following sorption of $10^{-5} \mathrm{M}$ Pb on $3 \mathrm{~g} / \mathrm{L}$ of samples $2800 \mathrm{D}, 2960 \mathrm{D}$, and 3270D.

Although the Cs ion was expected to form a smaller hydrated ion, because of low charge and big crystallographic radius (1.69 $\AA$ ) (Bohn et al., 1985), Cs sorption results showed generally lower sorption affinity for these samples compared to $\mathrm{Pb}$, except in cases where cation exchange sites were clearly abundant. Under these conditions, all three cations were quantitatively removed from solution. These results can be explained by the high hydrolysis or deprotonation constant of $\mathrm{Pb}$, which refers to the loss of $\mathrm{H}^{+}$from water ligands associated with central cations (Bohn et al., 1985). Due to the high hydrolysis constant, $\mathrm{Pb}$ can easily lose part of its primary hydration shell, especially at high $\mathrm{pH}$. The hydrolyzed ions will initially form strong inner-sphere complexes with oxide surfaces and eventually lead to formation of polynuclear complexes or surface precipitates.

The formation of $\mathrm{Pb}$ inner-sphere complexes on aluminum oxides and Rainier Mesa tuffs at high $\mathrm{pH}$ has been reported in previous XAS studies (Bargar et al., 1997; Um, 2001; Um and Papelis, 2003). Similarly, Pb sorption on Frenchman Flat samples was considered to be relatively strong compared to $\mathrm{Sr}$ and $\mathrm{Cs}$, which are thought to form outer-sphere complexes only. Based on desorption experiments, $\mathrm{Pb}$ was expected to be immobile in the subsurface environment, unless there were a substantial change in groundwater solution composition. In addition, these desorption results were in good agreement with the previously conducted sorption experiments, and also consistent with strong sorption of $\mathrm{Pb}$, moderate sorption of $\mathrm{Cs}$, and relatively weak sorption of Sr on Frenchman Flat volcanic tuffs.

\section{Sorption/Desorption Isotherms}

Most transport model codes that attempt to incorporate chemical reactions to predict the migration of contaminants in the subsurface environment require parameters describing the partitioning of the contaminants between the aqueous and solid interfaces. Although sorption 
modeling based on the surface complexation paradigm has been extensively used during the last two decades, most transport models still use parameters derived from isotherms to incorporate surface chemical reactions into numerical models.

Sorption/desorption isotherms represent the amount of adsorbate sorbed on adsorbents and desorbed from the solids, respectively. The amount of solute sorbed onto the sorbent can be measured by the mass difference between the initial and final metal concentration in solution. Several isotherms have been developed and used over the years; the most commonly used isotherms are the linear, Freundlich, and Langmuir isotherms. The linear distribution coefficient or $\mathrm{K}_{\mathrm{d}}$ is a function of the properties of the solid, the solute concentration, and the geochemical conditions in the background electrolyte solution. Because of its simplicity and straightforward incorporation in transport models, $\mathrm{K}_{\mathrm{d}} \mathrm{S}$ are still widely used in transport codes to incorporate partitioning reactions. Because of the generally reversible sorption behavior of organic contaminants, the distribution coefficients are typically more appropriate to predict organic contaminant sorption on aquifer materials.

For inorganic contaminants, sometimes the Freundlich or Langmuir isotherms provide better results compared to linear isotherms. The Freundlich isotherm is the oldest nonlinear sorption isotherm, which has been widely used to describe the sorption of solutes on soils (Travis and Etnier, 1981). Equilibrium sorption data are first linearized: if the value of $1 / \mathrm{n}$ in the Freundlich isotherm is equal to 1.0, the Freundlich isotherm reduces to the linear isotherm. If the value of $1 / \mathrm{n}$ is not equal to 1.0 , sorption is considered to be nonlinear and equilibrium sorption data can be plotted on a log-log scale resulting in a straight line, in turn resulting in determination of the Freundlich constant $\left(\mathrm{K}_{\mathrm{F}}\right)$ and a measure of nonlinearity $(1 / \mathrm{n})$ (Drever, 1997; Sloop, 1998). The nonlinearity of sorption is frequently correlated to the heat of a sorption reaction and can therefore provide some limited information about the sorption process (Weber and DiGiano, 1996). Within limited conditions, such as low solute concentration, the nonlinearity of the Freundlich and Langmuir isotherms is negligible so that the linear isotherm can be used without substantial loss of accuracy. Linear distribution coefficients $\left(\mathrm{K}_{\mathrm{d}}\right)$ for sorption and desorption isotherms were determined by the ratio of the concentration of sorbed ions (sorbed metal ion mass per mass of sorbent, $\mathrm{M} \mathrm{M}^{-1}$ ) to the equilibrium metal ion concentration in solution $\left(\mathrm{M} \mathrm{L}^{-3}\right)$. Sorption and desorption isotherms were obtained by changing metal and solid concentrations at fixed $\mathrm{pH}(\mathrm{pH} \approx 8.0)$. Sorption isotherms were prepared after 48-hour equilibration time. Because 14 days were shown to be enough to reach equilibrium based on the previously conducted desorption experiments, the metal concentration released in the solution was measured after 14 days following fresh solution introduction.

The linear sorption/desorption and Freundlich sorption isotherms for $\mathrm{Sr}$ are shown in Figures 9 and10, respectively. Desorption distribution coefficients for $\mathrm{Sr}, \mathrm{K}_{\mathrm{ds}(\mathrm{des})}$ were higher than sorption $\mathrm{K}_{\mathrm{d}(\mathrm{s})}$ for both the 2800D and 2960D samples, indicating initially sorbed $\mathrm{Sr}$ ions did not desorb reversibly. The $\mathrm{Sr}$ desorption $\mathrm{K}_{\mathrm{d}}$ was found to be higher than the sorption $\mathrm{K}_{\mathrm{d}}$ only in sample 3270D. Because most Sr ions sorbed on surface hydroxyl sites of sample 3270D by weak electrostatic attraction or hydrogen bond, the initially sorbed Sr would probably desorb from the solid surface by competition from background electrolyte cations. Sorption of Sr would be considered at least partially reversible under these conditions. Freundlich isotherms were also obtained and the calculated Freundlich parameters were compared to $\mathrm{K}_{\mathrm{d}} \mathrm{s}$. Calculated $\mathrm{K}_{\mathrm{d}}$ and Freundlich $\mathrm{K}_{\mathrm{F}}$ for $\mathrm{Sr}$ are given in Tables 3 and 4, respectively. There was no significant difference between $\mathrm{K}_{\mathrm{d}}$ and $\mathrm{K}_{\mathrm{F}}$. The Freundlich parameters were used in the pore diffusion model. 


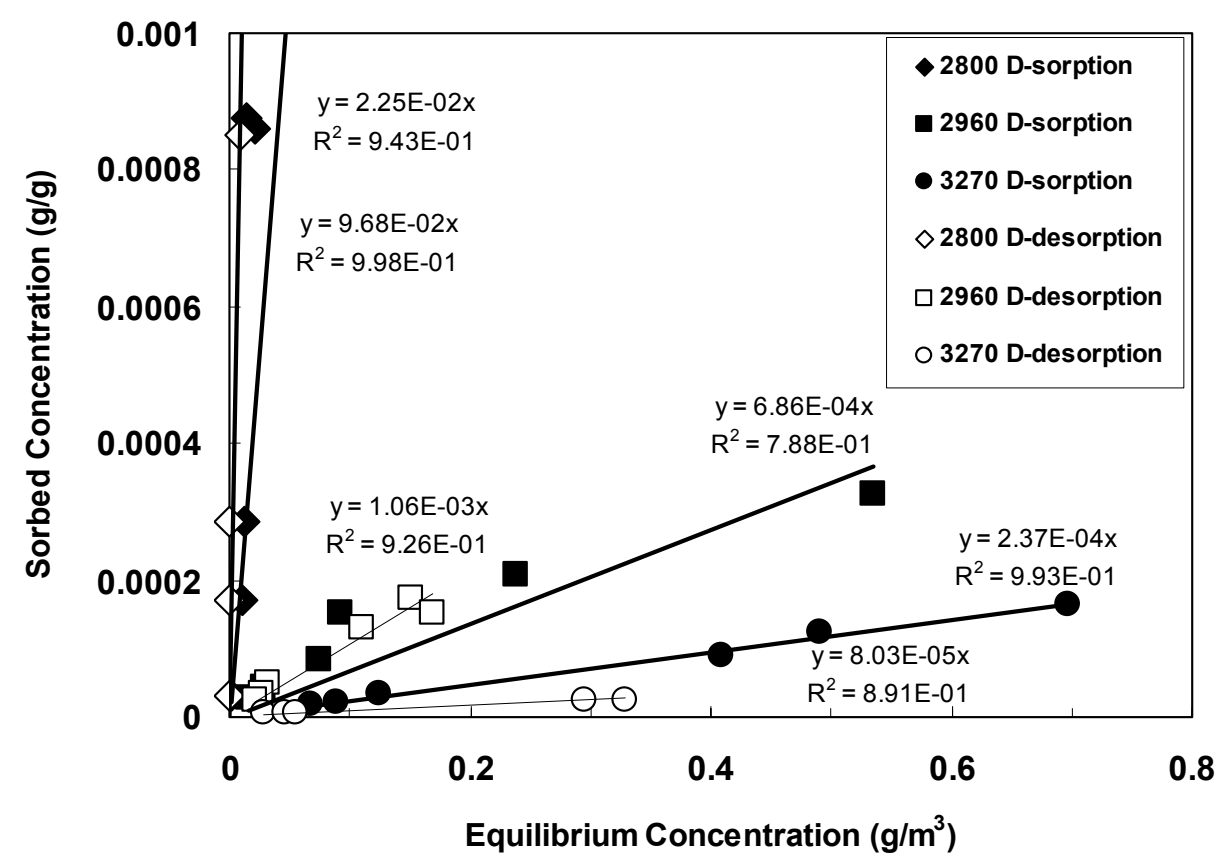

Figure 9. Linear sorption and desorption Sr isotherms for samples 2800D, 2960D, and 3270D.

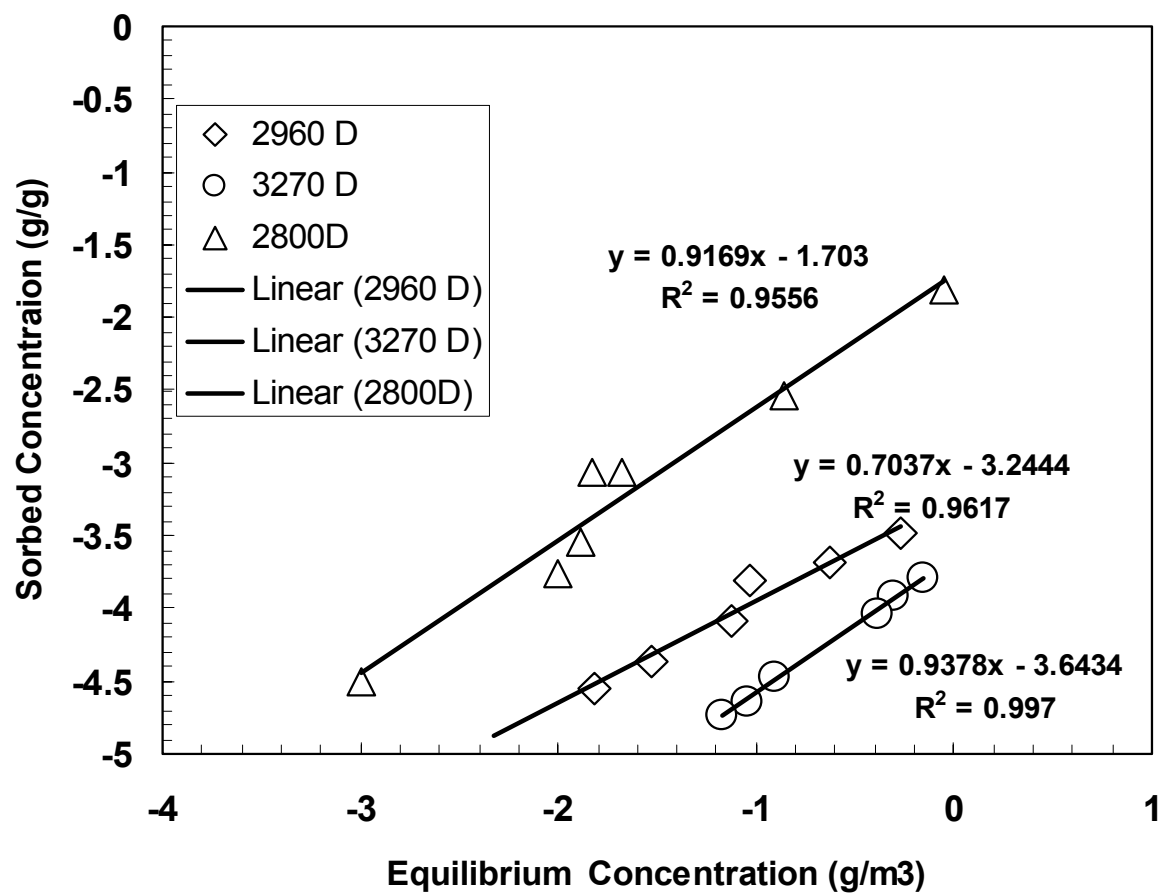

Figure 10. Freundlich Sr isotherms for samples 2800D, 2960D, and 3270D. 
Table 3. Linear sorption/desorption isotherms for $\mathrm{Sr}, \mathrm{Cs}$, and $\mathrm{Pb}$.

\begin{tabular}{llllll}
\hline Core samples & Metal & $\mathrm{K}_{\mathrm{d}(\mathrm{s})}\left(\mathrm{m}^{3} \mathrm{~g}^{-1}\right)^{*}$ & $\mathrm{R}^{2 \#}$ & $\mathrm{~K}_{\mathrm{d}(\mathrm{des})}\left(\mathrm{m}^{3} \mathrm{~g}^{-1}\right)^{+}$ & $\mathrm{R}^{2 \#}$ \\
\hline ER5/3/2-2800D & $\mathrm{Sr}$ & $2.25 \times 10^{-2}$ & 0.943 & $9.68 \times 10^{-2}$ & 0.998 \\
& $\mathrm{Cs}$ & $2.78 \times 10^{-3}$ & 0.999 & $3.08 \times 10^{-3}$ & 0.986 \\
& $\mathrm{~Pb}$ & $1.29 \times 10^{-2}$ & 0.990 & $5.70 \times 10^{-2}$ & 0.984 \\
ER5/3/2-2960D & $\mathrm{Sr}$ & $6.86 \times 10^{-4}$ & 0.788 & $1.06 \times 10^{-3}$ & 0.926 \\
& $\mathrm{Cs}$ & $6.72 \times 10^{-4}$ & 0.992 & $9.95 \times 10^{-4}$ & 0.993 \\
& $\mathrm{~Pb}$ & $8.62 \times 10^{-3}$ & 0.974 & $1.02 \times 10^{-2}$ & 0.994 \\
ER5/3/2-3270D & $\mathrm{Sr}$ & $2.37 \times 10^{-4}$ & 0.993 & $8.03 \times 10^{-5}$ & 0.891 \\
& $\mathrm{Cs}$ & $8.70 \times 10^{-4}$ & 0.977 & $1.43 \times 10^{-3}$ & 0.925 \\
& $\mathrm{~Pb}$ & $5.50 \times 10^{-3}$ & 0.964 & $6.90 \times 10^{-3}$ & 0.994 \\
\hline
\end{tabular}

* Sorption $\mathrm{K}_{\mathrm{d}}$

${ }^{+}$Desorption $\mathrm{K}_{\mathrm{d}}$

${ }^{\#}$ Correlation coefficient

Table 4. Freundlich isotherm parameters for $\mathrm{Sr}, \mathrm{Cs}$, and $\mathrm{Pb}$ at $\mathrm{pH}=8.0$.

\begin{tabular}{lllll}
\hline Core samples & Metal & $\mathrm{K}_{\mathrm{f}}(\mathrm{g} / \mathrm{g}) /\left(\mathrm{g} / \mathrm{m}^{3}\right)^{1 / \mathrm{n}}$ & $1 / \mathrm{n}(-)$ & $\mathrm{r}^{{ }^{*}}$ \\
\hline ER5/3/2-2800D & $\mathrm{Sr}$ & $1.98 \times 10^{-2}$ & 0.917 & 0.956 \\
& $\mathrm{Cs}$ & $3.15 \times 10^{-3}$ & 1.065 & 0.998 \\
ER5/3/2-2960D & $\mathrm{Pb}$ & $1.41 \times 10^{-2}$ & 1.035 & 0.875 \\
& $\mathrm{Sr}$ & $5.70 \times 10^{-4}$ & 0.704 & 0.962 \\
& $\mathrm{Cs}$ & $1.75 \times 10^{-3}$ & 1.200 & 0.928 \\
ER5/3/2-3270D & $\mathrm{Pb}$ & $1.09 \times 10^{-2}$ & 1.108 & 0.872 \\
& $\mathrm{Sr}$ & $2.27 \times 10^{-4}$ & 0.938 & 0.997 \\
& $\mathrm{Cs}$ & $7.60 \times 10^{-4}$ & 0.939 & 0.988 \\
& $\mathrm{~Pb}$ & $2.80 \times 10^{-3}$ & 0.730 & 0.954 \\
\hline
\end{tabular}

${ }^{*}$ Correlation coefficient

Linear sorption/desorption and Freundlich sorption isotherms for $\mathrm{Cs}$ and $\mathrm{Pb}$ are shown in Figures 11 to 14. The desorption $\mathrm{K}_{d} \mathrm{~S}$ for $\mathrm{Cs}$ and $\mathrm{Pb}$ were higher compared to sorption $\mathrm{K}_{d} \mathrm{~S}$, indicating strong sorption affinities of $\mathrm{Cs}$ and $\mathrm{Pb}$ ions for these samples. Based on these sorption/desorption experiments, $\mathrm{Cs}$ and $\mathrm{Pb}$ sorption was expected to be irreversible so that initially sorbed $\mathrm{Cs}$ and $\mathrm{Pb}$ on Frenchman Flat volcanic tuff materials would not easily desorb under the same geochemical conditions. A small amount of desorbed $\mathrm{Cs}$ and $\mathrm{Pb}$ was consistent with the previously performed sorption and desorption experiments, suggesting stronger sorption of $\mathrm{Cs}$ and $\mathrm{Pb}$ on these samples compared to Sr. The calculated distribution coefficients of sorption/desorption and Freundlich parameters for $\mathrm{Cs}$ and $\mathrm{Pb}$ are also given in Tables 3 and 4 . The results were similar between $\mathrm{K}_{\mathrm{d}}$ and $\mathrm{K}_{\mathrm{F}}$, indicating that linear isotherms could be used for modeling purposes under these specific conditions. 


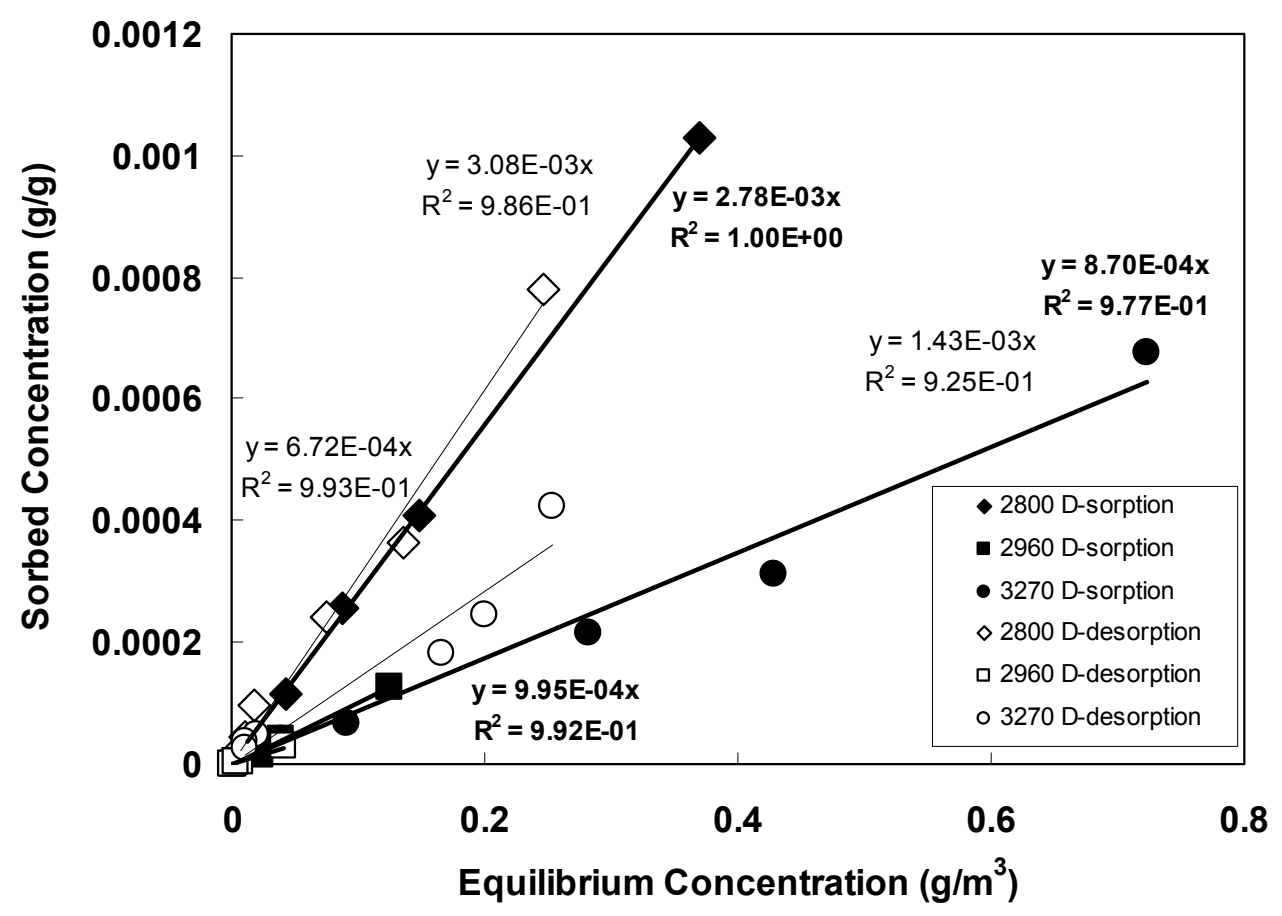

Figure 11. Linear sorption and desorption Cs isotherms for samples 2800D, 2960D, and 3270D.

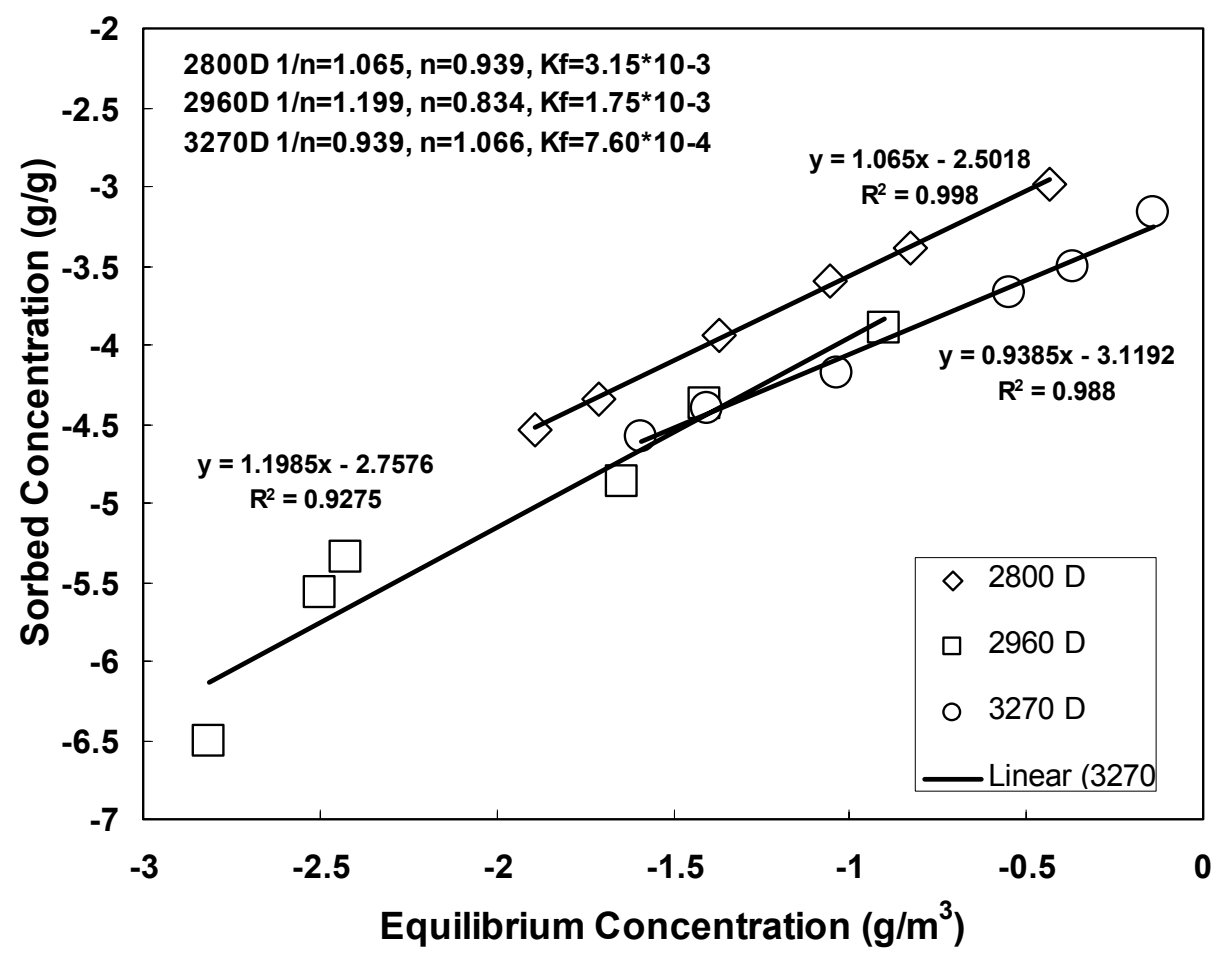

Figure 12. Freundlich Cs isotherms for samples 2800D, 2960D, and 3270D. 


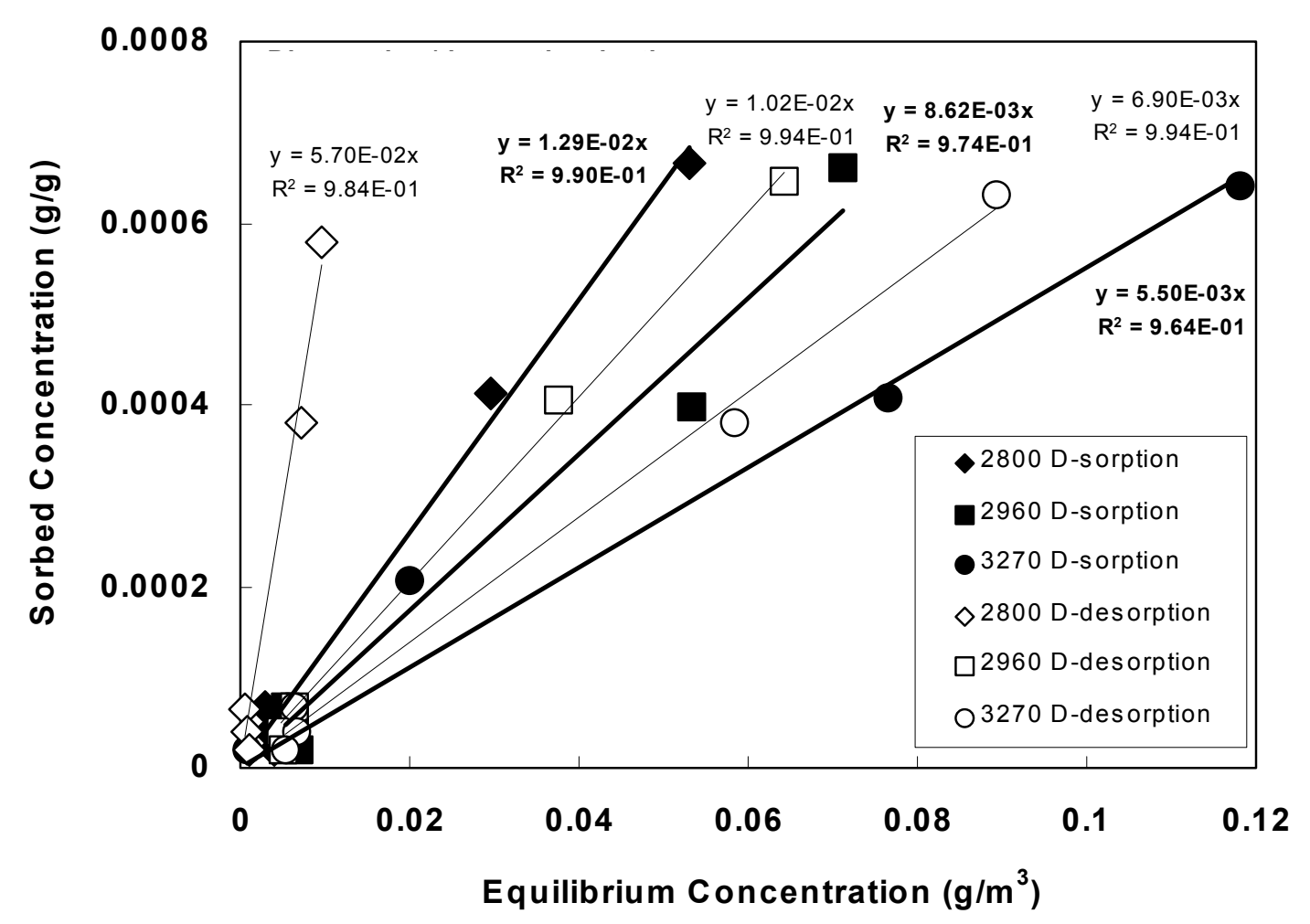

Figure 13. Linear sorption and desorption $\mathrm{Pb}$ isotherms for samples 2800D, 2960D, and 3270D.

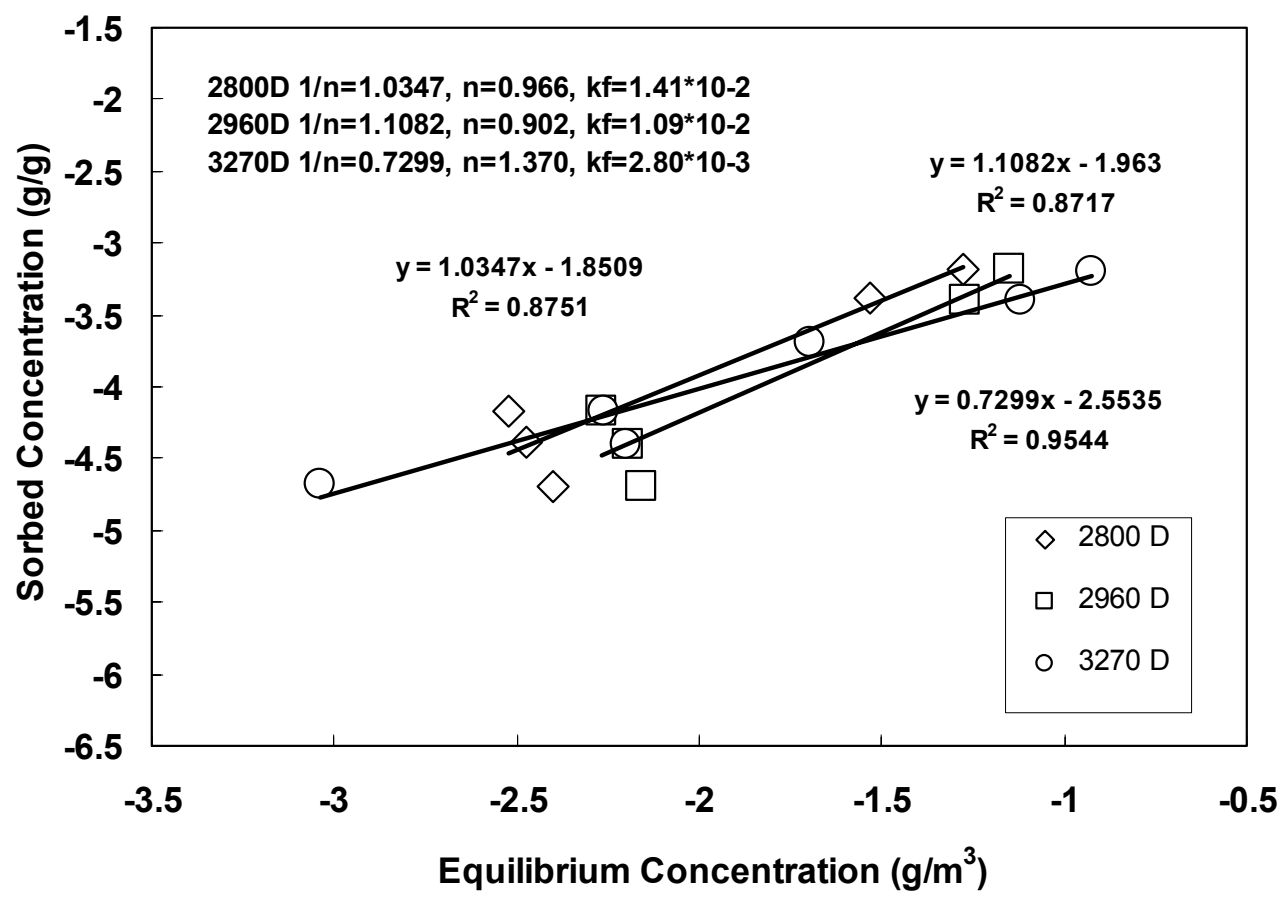

Figure 14. Freundlich $\mathrm{Pb}$ isotherms for samples 2800D, 2960D, and 3270D. 


\section{Rate of Uptake Experiments and Modeling}

Rate of uptake experiments with the three different cations and the three volcanic tuff samples were conducted to find out if diffusion processes controlled the rate of metal uptake from solution. The rate data are presented as fractional metal uptake versus time. In summary, the results indicated that all three metals $(\mathrm{Sr}, \mathrm{Cs}$, and $\mathrm{Pb})$ reached equilibrium the fastest in the case of sample 2800D. Although $\mathrm{Pb}$ also exhibited fast sorption uptake by samples 2960D and 3270D, Sr and Cs did not reach equilibrium even after 30 days. Discussion of these results will be presented below. Based on these results, diffusion-controlled sorption appeared to control the uptake of Cs and Sr by samples 2960D and 3270D and particles of the dimension used (approximately $2 \mathrm{~mm}$ ). To test this hypothesis, the pore diffusion model was used to fit the observed rate of metal uptake.

The pore diffusion model uses Fick's second law and assumes solute diffusion into spherical porous aggregates in a well-stirred reactor of limited volume, fixed initial concentration, diffusion only in the aqueous phase, and sorption represented by the Freundlich adsorption isotherm (Fuller et al., 1993). The computer code used to optimize the model-fitting parameters was obtained from G.Curtis (U.S. Geological Survey, Menlo Park, CA). The code iterates until the sum of the square of the difference between the measured data and calculated values reaches a minimum value. The Freundlich isotherm parameters $\left(K_{F}\right.$ and $\left.1 / n\right)$ were obtained from previously obtained sorption isotherms and were fixed during modeling. The adjustable parameters during fitting were $\mathrm{D}_{\text {app }}$ (apparent diffusion coefficient), $\mathrm{F}_{\text {eq }}$ (fraction of instantaneous equilibrium sorption sites relative to total available sites), and $\mathrm{n}$ (porosity).

The Sr uptake data and modeling results are shown in Figure 15. The pore diffusion model fit the Sr uptake by sample 3270D well and by sample 2960D reasonably well, suggesting that diffusion controlled the sorption uptake of $\mathrm{Sr}$ on these materials. Because the obtained $\mathrm{F}_{\mathrm{eq}}$ value was negligible, one must hypothesize that $\mathrm{Sr}$ ions diffused inside porous materials and reacted with sorption sites present in either pore walls or micropores. This assumption appears to be reasonable given the specific surface area and composition of these adsorbents on one hand, and the ability of Sr to form outer-sphere complexes only. It should also be noted that under the experimental conditions used, $\mathrm{Sr}$ ions remained in solution, even after 30 days, implying that $\mathrm{Sr}$ ions would have to diffuse inside particle pores before sorption equilibrium could be established.

Calculated diffusion parameters are given in Table 5. Because Sr was a reactive element, apparent diffusivity rather than effective diffusivity was obtained from the pore diffusion model using least-squares fitting. Apparent diffusivities and porosities were estimated from model simulations and appeared to be reasonable. The pore diffusion model, however, did not fit very well the data of Sr uptake by sample 2800D, suggesting that under the specific experimental conditions, diffusion was not the primary controlling mechanism. Because the 2800D sample had high cation exchange capacity and high surface area, as a result of the high percentage of zeolites present in this sample, Sr sorption was probably controlled by ion-exchange on abundant cation exchange sites, located close to the external surface area of the particles. Under these conditions, the sorption process is not controlled by diffusion. The fact that essentially complete removal was accomplished within two days indicates that enough sorption sites were readily available for Sr uptake. Under these conditions, diffusion and sorption of Sr into deeper particle regions would not be required. In addition, because of strong Sr sorption on sample 2800D, fitting results were not improved when $\mathrm{F}_{\text {eq }}$ was fixed from 0.01 to 1 . The apparent diffusivity of $\mathrm{Sr}$ was calculated by fixing the value of $F_{\text {eq }}$ at 0.8 . 


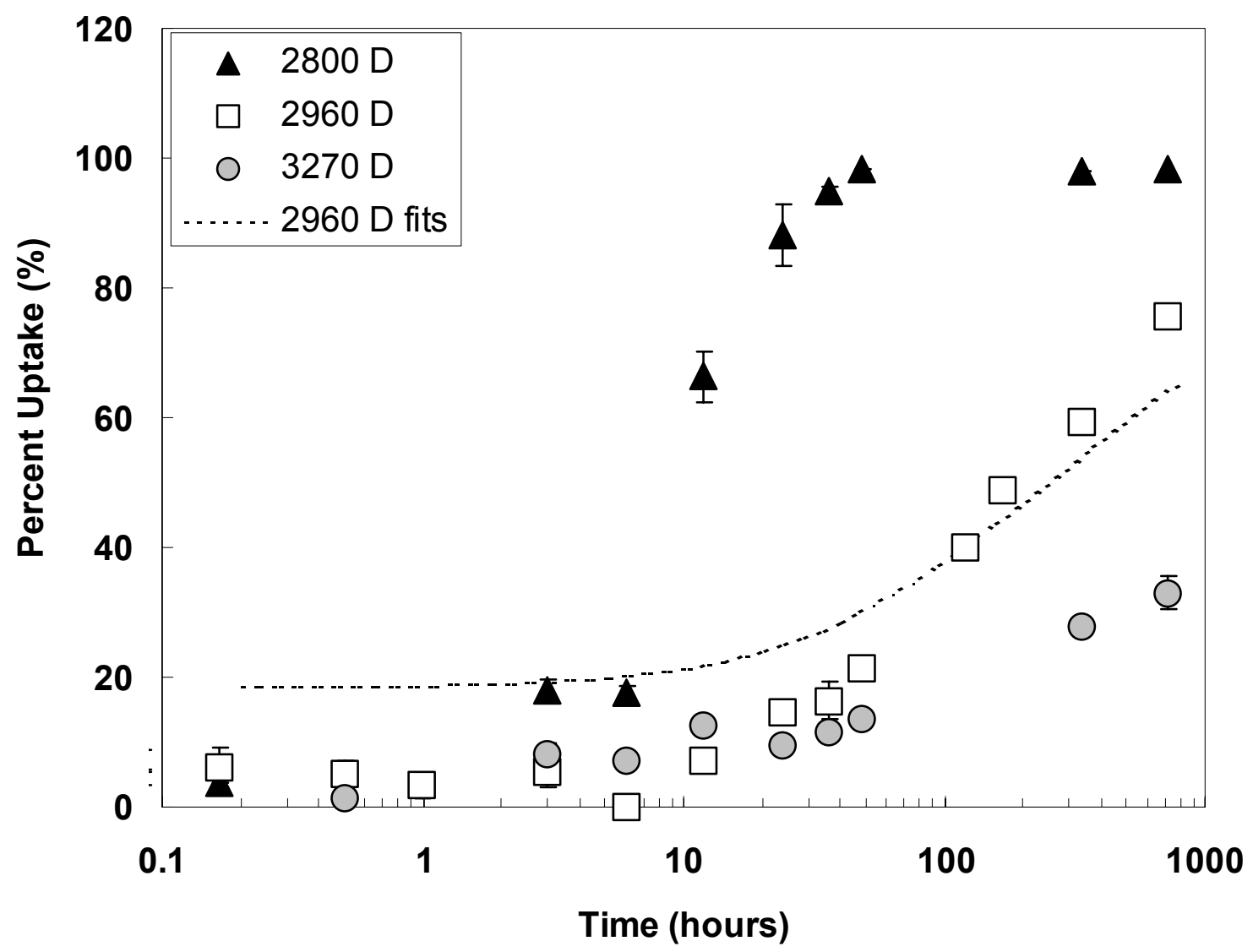

Figure 15. Modeling the rate of Sr uptake by samples 2800D, 2960D, and 3270D.

Table 5. Pore diffusion model results.

\begin{tabular}{lllll}
\hline Core samples & Metal & $\mathrm{D}_{\text {app }}\left(\mathrm{m}^{2} \mathrm{~s}^{-1}\right)$ & $\mathrm{F}_{\mathrm{eq}}$ & $\mathrm{n}(-)$ \\
\hline ER5/3/2-2800D & $\mathrm{Sr}$ & $2.46 \times 10^{-14}$ & $0.8^{*}$ & 0.28 \\
& $\mathrm{Cs}$ & $1.96 \times 10^{-13}$ & $0.8^{*}$ & 0.24 \\
$\mathrm{~Pb}$ & $1.26 \times 10^{-13}$ & $0.8^{*}$ & 0.20 \\
& & $1.26 \times 10^{-13}$ & $0.3^{*}$ & $0.20^{*}$ \\
ER5/3/2-2960D & & $1.26 \times 10^{-13}$ & $0.1^{*}$ & $0.20^{*}$ \\
& $\mathrm{Sr}$ & $1.26 \times 10^{-13}$ & $0.01^{*}$ & $0.20^{*}$ \\
& $\mathrm{Cs}$ & $7.55 \times 10^{-11}$ & 0.01 & 0.22 \\
& $\mathrm{~Pb}$ & $1.99 \times 10^{-11}$ & 0.01 & 0.18 \\
$\mathrm{ER} 5 / 3 / 2-3270 \mathrm{D}$ & $1.26 \times 10^{-13}$ & $0.8^{*}$ & 0.20 \\
& & $1.25 \times 10^{-13}$ & $0.3^{*}$ & 0.20 \\
& & $2.20 \times 10^{-9}$ & $0.1^{*}$ & 0.06 \\
& $\mathrm{Sr}$ & $8.56 \times 10^{-9}$ & $0.01^{*}$ & 0.10 \\
& $\mathrm{Cs}$ & $1.12 \times 10^{-10}$ & 0.01 & 0.12 \\
& $\mathrm{~Pb}$ & $1.91 \times 10^{-9}$ & 0.01 & 0.18 \\
& & $8.26 \times 10^{-13}$ & $0.8^{*}$ & 0.20 \\
& & $1.62 \times 10^{-9}$ & $0.3^{*}$ & 0.10 \\
& & $1.35 \times 10^{-9}$ & $0.1^{*}$ & 0.10 \\
\hline
\end{tabular}

\footnotetext{
${ }^{*}$ Parameter was fixed during model fits.
} 
The results of Cs experimental uptake experiments and modeling were similar to $\mathrm{Sr}$ (Figure 16). The sorption uptake of Cs by samples 2960D and 3270D was predicted by the pore diffusion model reasonably well, although the model failed to match the slow Cs uptake in the beginning of the experiment, especially for sample $3270 \mathrm{D}$, and it appeared to overpredict the sorption at later stages for sample 2960D. These results suggest that the rate of Cs uptake under these conditions may not be controlled by diffusion alone. As in the case of $\mathrm{Sr}$, Cs sorption uptake by $2800 \mathrm{D}$ was also not predicted very well by the diffusion model. Because of abundance of cation exchange sites on this sample and instantaneous equilibrium of $\mathrm{Cs}$ on either permanent charge sites or surface hydroxyl sites, Cs ions were not required to diffuse inside the interior of the particle. Sorption of Cs on 2800D was, therefore, not considered to be controlled by diffusion, under these specific experimental conditions. Because the equilibrium Cs uptake on samples 2960D and 3270D was less than 100 percent, even after sufficient equilibration time, diffusion and sorption of Cs in the interior of the particles must be assumed.

Uptake of $\mathrm{Pb}$ ions was fast by all three Frenchman Flat samples. The experimental $\mathrm{Pb}$ rate of uptake data by sample 2800D and the model simulations are shown in Figure 17. Because of strong binding and the fast sorption reactions involved, the diffusion model did not fit very well the $\mathrm{Pb}$ sorption data. Although several values of $\mathrm{F}_{\text {eq }}$ were tried, from 0.01 to 0.8 , the model results did not improve. The results of Pb sorption uptake by 2960D and 3270D are shown in Figures 18 and 19. The diffusion model results improved when a small $\mathrm{F}_{\mathrm{eq}}$ value was used (e.g., $0.01)$. However, the calculated apparent diffusivity was even faster than molecular diffusivity of $\mathrm{Pb}\left(1.41 \times 10^{-9} \mathrm{~m}^{2} \mathrm{~s}^{-1}\right)$ in solution. These results point to the conclusion that the observed rate of $\mathrm{Pb}$ uptake was not controlled by diffusion.

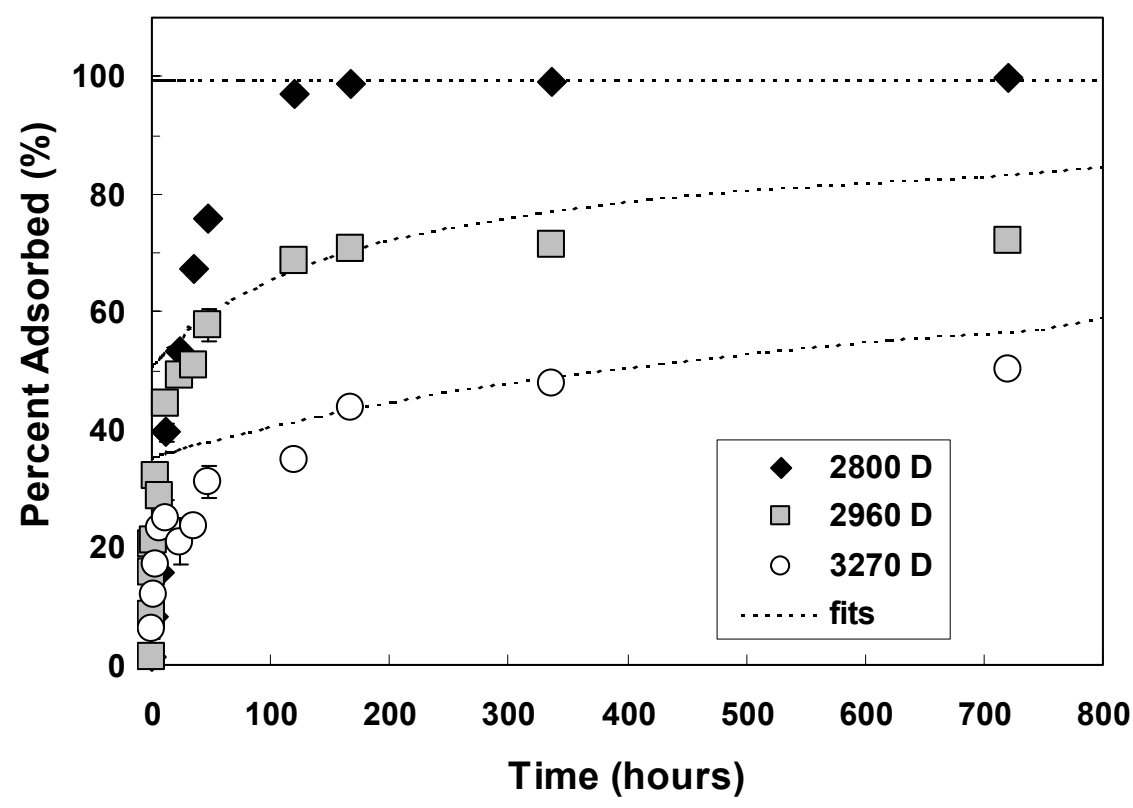

Figure 16. Modeling the rate of Cs uptake by samples 2800D, 2960D, and 3270D. 


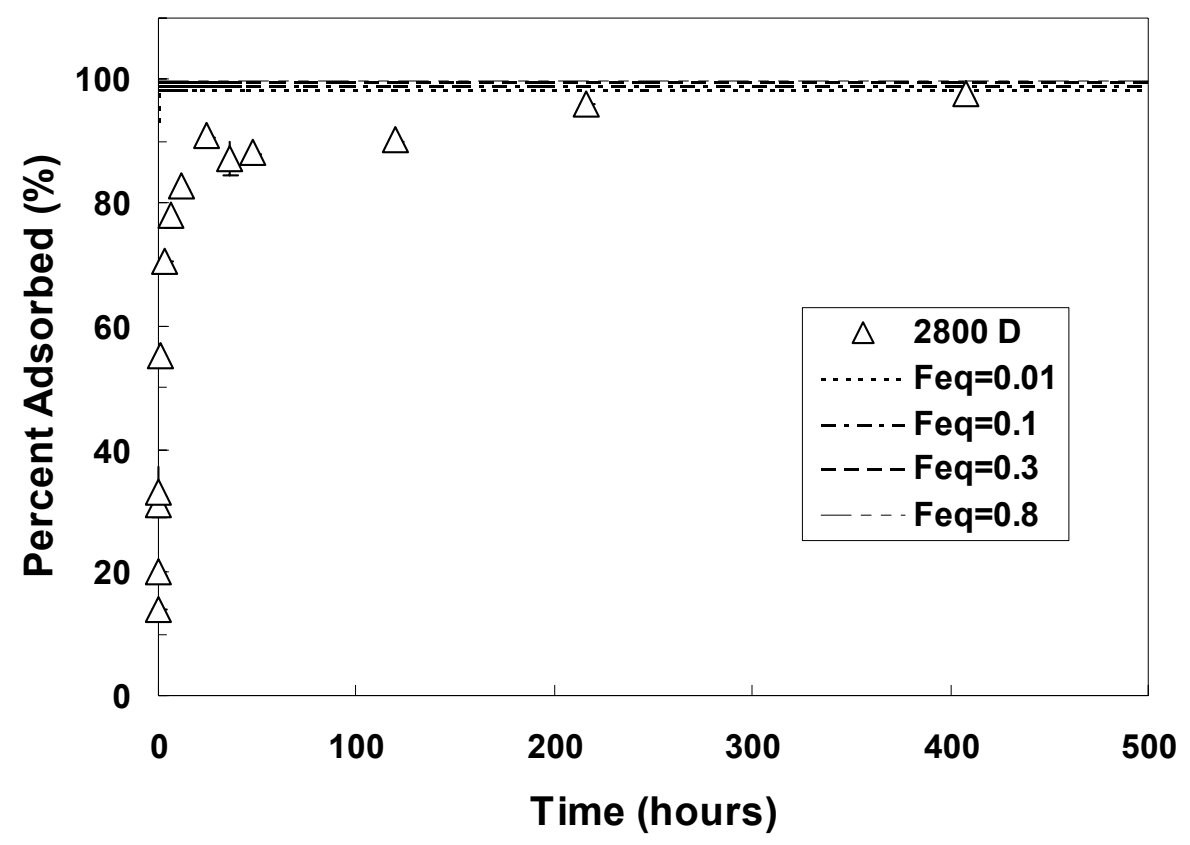

Figure 17. Modeling the rate of $\mathrm{Pb}$ uptake by sample 2800D.

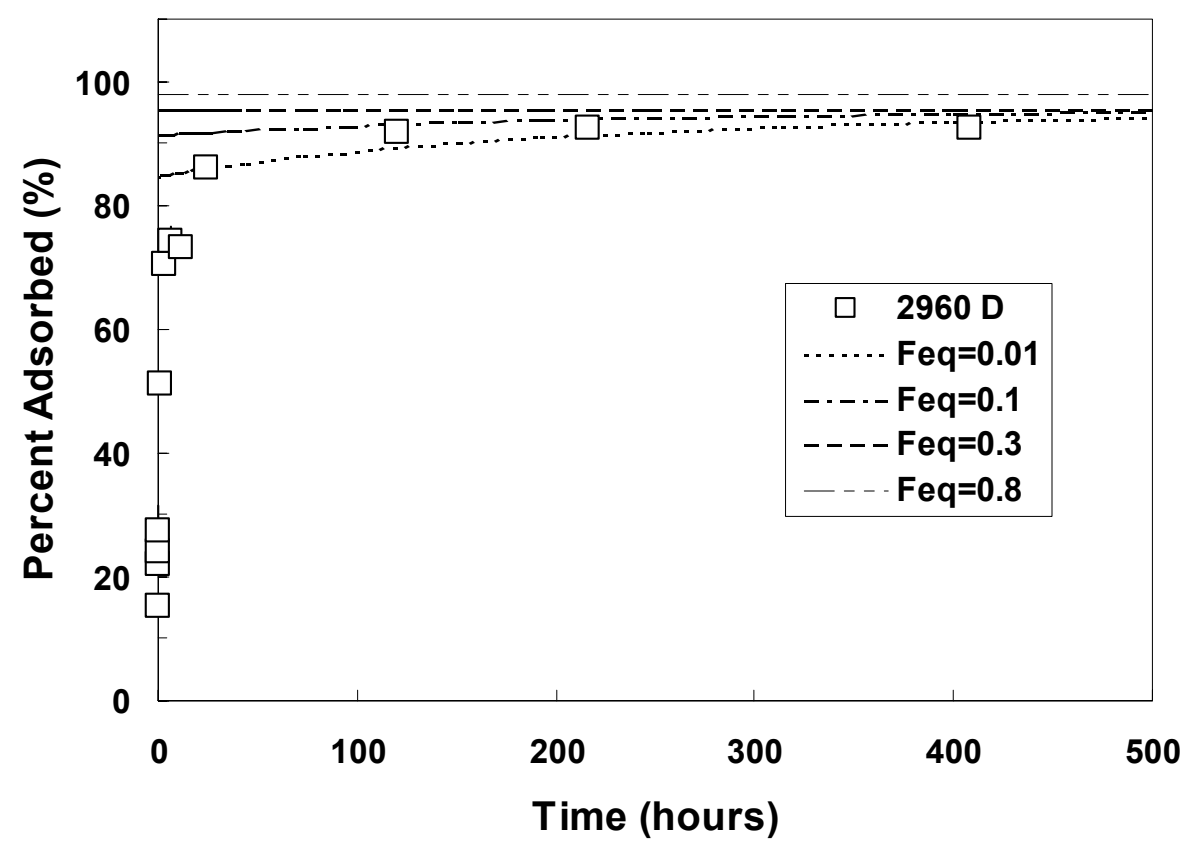

Figure 18. Modeling the rate of $\mathrm{Pb}$ uptake by sample 2960D. 


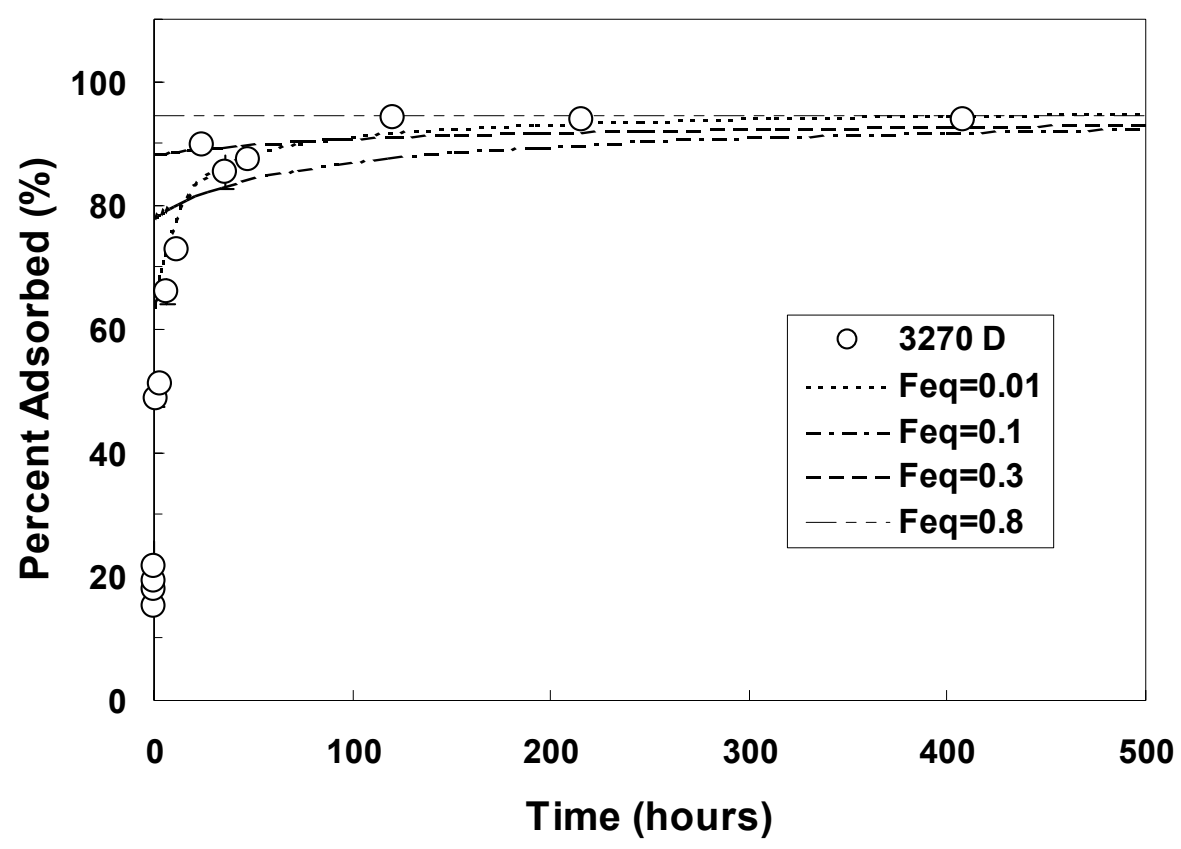

Figure 19. Modeling the rate of $\mathrm{Pb}$ uptake by sample 3270D.

\section{XPS Experiments}

The objective of the XPS experiments was to compare the macroscopic sorption and diffusion experiments to the spectroscopic information obtained from the characterization of these surfaces before and after sorption and diffusion. The major advantage of the XPS experiments compared to the macroscopic experiments is that specific information on chemical composition and chemical state of a surface can be obtained. Information about surface composition and chemical state, of course, is critical in any surface study. In addition, using XPS, information about specific areas of a sample can be obtained, depending on the spot size of analysis used.

For this work, XPS data were collected on the Frenchman Flat powders before the sorption experiments and after. Two types of general information were collected and will be discussed below: binding energies (BE) of elements present and information on elemental abundance. The information on BE is representative of the chemical state of the element and can provide valuable information regarding oxidation state and coordination environment. The elemental composition, besides being useful information by itself in terms of interpreting macroscopic sorption experiments, can be used to estimate the surface coverage of sorbents and to distinguish between different types of sorption, including adsorption and surface precipitation.

For every sample, a broad survey spectrum was collected, in the 0 to $1100 \mathrm{eV}$ energy range, using a high-sensitivity but low-resolution data collection mode ( $1 \mathrm{eV} / \mathrm{step})$. The survey scan was then followed by narrower scans, typically 20 to $30 \mathrm{eV}$, with lower sensitivity but higher resolution $(0.065 \mathrm{eV} / \mathrm{step})$ for the elements of interest. These elements typically included the major cations anticipated or observed in the sample, including silicon ( $\mathrm{Si}$ ), aluminum (Al), sodium $(\mathrm{Na})$, potassium $(\mathrm{K})$, calcium $(\mathrm{Ca})$, magnesium $(\mathrm{Mg})$, iron $(\mathrm{Fe})$, manganese $(\mathrm{Mn})$, and the anion oxygen $(\mathrm{O})$. In addition, narrow scans of the sorbates of interest, strontium (Sr), cesium 
$(\mathrm{Cs})$, and lead $(\mathrm{Pb})$ were also included when analyzing sorption samples. A typical survey scan of a core sample, with major photoelectron peaks labeled, is shown in Figure 20. Narrow scans for individual elements are shown in the following section.

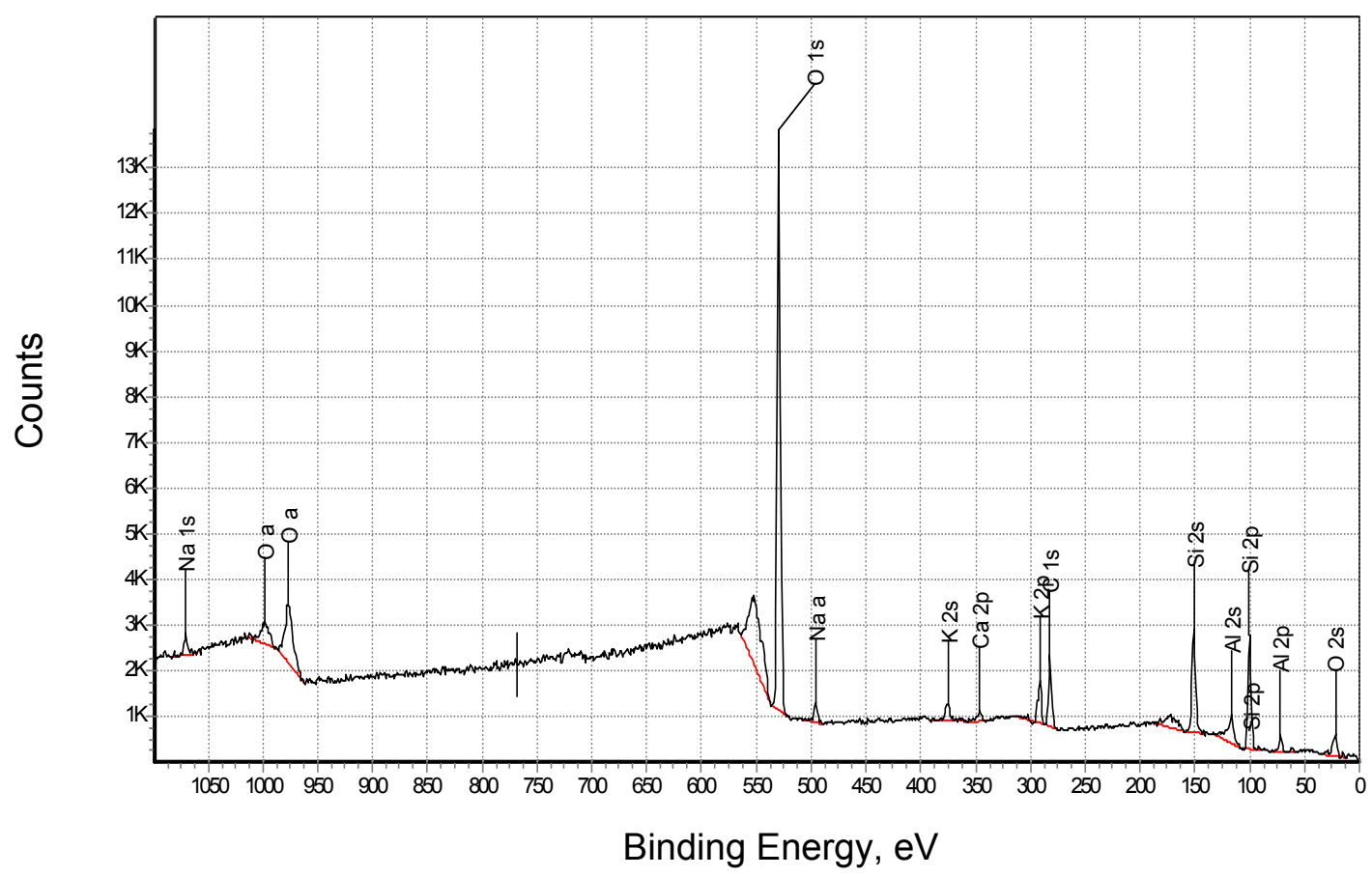

Figure 20. Typical XPS survey scan of a Frenchman Flat powder sample.

\section{Binding Energies}

The BE and corresponding chemical shift for all elements collected in narrow scan mode and for all samples analyzed are listed in Table 6 . The results are grouped together by sample and element. By comparing the measured BE to the BEs reported in the literature, one can draw conclusions about the chemical state and coordination environment of the element. The charge referencing for all narrow scans was made by the adventitious carbon method assuming the energy of the $\mathrm{C} 1 \mathrm{~s}$ line to be $284.6 \mathrm{eV}$.

A representative XPS spectrum of the Al 2p peak is shown in Figure 21. Average chemical shifts for most samples ranged between 1.10 and $1.75 \mathrm{eV}$, clustered mostly between 1.2 and $1.4 \mathrm{eV}$. The average chemical shift was around $1.3 \mathrm{eV}$ with very little variation between the three samples. These chemical shifts correspond to BEs ranging from 74.00 to $74.75 \mathrm{eV}$ with an average around $74.3 \mathrm{eV}$. These numbers are in good agreement with the quantitative mineralogy of these samples. For example, the BE for elemental Al is $72.9 \mathrm{eV}$, substantially lower than the measured BEs, whereas the Al in oxides and aluminosilicates corresponds to a BE of 74 to 75 . Specifically, the BE of albite, a very common feldspar, is $74.3 \mathrm{eV}$, in excellent agreement with the measured BE. The majority of samples 2960D and 3270D was comprised of feldspars. 
Table 6. Chemical shifts and binding energies $(\mathrm{eV})$ of major elements in powder samples.

\begin{tabular}{|c|c|c|c|c|c|c|c|c|c|c|c|c|c|c|c|c|c|c|c|c|}
\hline \multirow[b]{2}{*}{ SAMPLE } & \multicolumn{20}{|c|}{ XPS Line } \\
\hline & $\begin{array}{l}\text { Al 2p } \\
\text { Chem. } \\
\text { Shift } \\
\end{array}$ & $\begin{array}{l}\text { Adj. } \\
\text { BE }\end{array}$ & $\begin{array}{l}\text { Si } 2 p \\
\text { Chem. } \\
\text { Shift }\end{array}$ & $\begin{array}{l}\text { Adj. } \\
\text { BE }\end{array}$ & $\begin{array}{l}\text { O 1s } \\
\text { Chem. } \\
\text { Shift }\end{array}$ & $\begin{array}{l}\text { Adj. } \\
\text { BE }\end{array}$ & $\begin{array}{l}\mathrm{Na} 1 \mathrm{~s} \\
\text { Chem. } \\
\text { Shift }\end{array}$ & $\begin{array}{l}\text { Adj. } \\
\text { BE }\end{array}$ & $\begin{array}{l}\text { K 2p3 } \\
\text { Chem. } \\
\text { Shift }\end{array}$ & $\begin{array}{l}\text { Adj. } \\
\text { BE }\end{array}$ & $\begin{array}{l}\text { Ca 2p3 } \\
\text { Chem. } \\
\text { Shift }\end{array}$ & $\begin{array}{l}\text { Adj. } \\
\text { BE }\end{array}$ & $\begin{array}{l}\text { Fe } 2 p 3 \\
\text { Chem. } \\
\text { Shift }\end{array}$ & $\begin{array}{l}\text { Adj. } \\
\text { BE }\end{array}$ & $\begin{array}{l}\text { Sr 3d5 } \\
\text { Chem. } \\
\text { Shift }\end{array}$ & $\begin{array}{l}\text { Adj. } \\
\text { BE }\end{array}$ & $\begin{array}{l}\text { Cs 3d5 } \\
\text { Chem. } \\
\text { Shift }\end{array}$ & $\begin{array}{l}\text { Adj. } \\
\text { BE }\end{array}$ & $\begin{array}{l}\mathrm{Pb} 4 \mathrm{f} 7 \\
\mathrm{Chem} . \\
\text { Shift }\end{array}$ & $\begin{array}{l}\text { Adj. } \\
\text { BE }\end{array}$ \\
\hline \multicolumn{21}{|l|}{ ER5/3/2 2800D } \\
\hline Powder & 1.00 & 74.00 & 3.49 & 102.49 & -0.11 & 531.89 & 0.86 & 1072.86 & -0.20 & 293.80 & 1.48 & 348.48 & 4.22 & 714.22 & & & & & & \\
\hline Powder w/10E-5 M Cs & 1.18 & 74.18 & 3.72 & 102.79 & 0.14 & 532.14 & 1.08 & 1073.08 & 0.02 & 294.02 & 1.62 & 348.62 & 2.86 & 712.86 & & & & & & \\
\hline Powder w/10E-5 M Pb & 1.75 & 74.75 & 4.46 & 103.46 & 1014 & 533.14 & & & -0.09 & 293.91 & & & 2.80 & 712.80 & & & & & & \\
\hline Powder w/10E-5 M Sr & 1.32 & 74.32 & 3.75 & 102.75 & 0.27 & 532.27 & 1.25 & 1073.25 & 0.36 & 294.36 & 1.87 & 348.87 & 1.80 & 711.80 & & & & & & \\
\hline \multicolumn{21}{|l|}{ ER5/3/2 2960D } \\
\hline Powder & 1.21 & 74.21 & 3.70 & 102.70 & 0.05 & 532.05 & 0.49 & 1072.49 & -0.67 & 293.33 & 1.20 & 348.2 & 1.91 & 711.91 & & & & & & \\
\hline Powder w/10E-5 M Cs & 1.40 & 74.40 & 3.84 & 102.84 & 0.28 & 532.28 & 0.83 & 1072.83 & -0.46 & 293.54 & 1.48 & 348.48 & 2.52 & 712.52 & & & -0.76 & 725.24 & & \\
\hline Powder w/10E-5 M Pb & 1.10 & 74.10 & 3.54 & 102.54 & 0.13 & 532.13 & 0.73 & 1072.73 & -0.86 & 293.14 & & & 2.04 & 712.04 & & & & & & \\
\hline Powder w/10E-5 M Sr & 1.39 & 74.39 & 3.82 & 102.82 & 0.30 & 532.30 & 0.84 & 1072.84 & -0.43 & 293.57 & 1.64 & 348.64 & 1.90 & 711.90 & 1.77 & 134.77 & & & & \\
\hline \multicolumn{21}{|l|}{ ER5/3/2 3270D } \\
\hline Powder & 1.18 & 74.18 & 3.79 & 102.79 & 0.06 & 532.06 & 0.41 & 1072.41 & -0.59 & 293.41 & 1.45 & 348.45 & 2.71 & 712.71 & & & & & & \\
\hline Powder w/10E-5 M Cs & 1.31 & 74.31 & 3.84 & 102.84 & 0.25 & 532.25 & 0.89 & 1072.89 & -0.55 & 293.45 & 1.52 & 348.62 & 2.89 & 712.89 & & & & & & \\
\hline Powder w/10E-5 M Pb & 1.62 & 74.62 & 4.08 & 103.08 & 0.50 & 532.50 & 1.08 & 1073.08 & -0.36 & 293.64 & & & 2.89 & 712.89 & & & & & & \\
\hline Powder w/10E-5 M Sr & 1.26 & 74.26 & 3.74 & 102.74 & 0.10 & 532.10 & 0.73 & 1072.73 & -0.57 & 293.43 & 0.99 & 347.99 & 2.36 & 712.36 & & & & & & \\
\hline
\end{tabular}




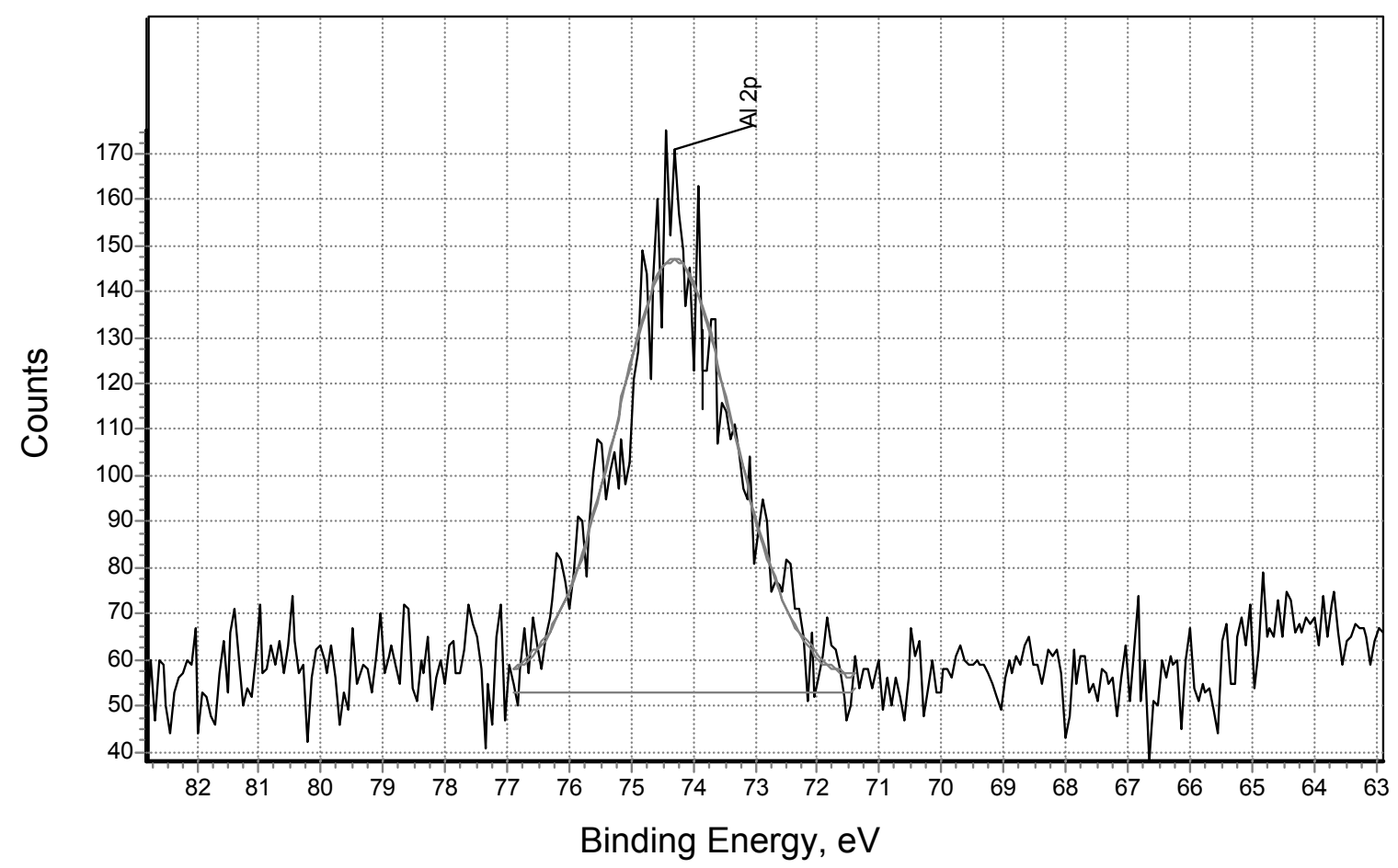

Figure 21. Typical Al 2p x-ray photoelectron spectrum in a Frenchman Flat powder sample.

A representative XPS spectrum of the Si $2 p$ peak is shown in Figure 22. Average chemical shifts for these samples ranged from 3.46 to $4.46 \mathrm{eV}$, with several clustered around 3.8 $\mathrm{eV}$, corresponding to Si $2 \mathrm{p}$ BEs from 102.46 to $103.46 \mathrm{eV}$, although the last value appears to be high. Average BE values were 102.7-102.9 eV. These values are in good agreement with BEs for silicates, which range between 102 and $103 \mathrm{eV}$, but they are too low for silica, with a Si $2 \mathrm{p} \mathrm{BE}$ of $103.5 \mathrm{eV}$. On the other hand, the Si $2 \mathrm{p}$ BEs for albite, mica, and kaolinite are 102.6, 102.4, and 103.0, respectively. The values measured, therefore, agree very well with a combination of mostly feldspars and other silicates and a smaller fraction of silica, again, in good agreement with the results of quantitative mineralogy performed on these samples.

A representative XPS spectrum of the $\mathrm{O} 1 \mathrm{~s}$ peak is shown in Figure 23. The $\mathrm{O} 1 \mathrm{~s}$ chemical shifts for all samples were very small, resulting essentially to a BE of $532 \mathrm{eV}$, an average value for several different oxygen containing compounds. The measured value, however, would be too high for metal oxides, that usually have BEs between 528 and $531 \mathrm{eV}$, and too low for silica, with a BE 532 to $533 \mathrm{eV}$. These results, therefore, are again consistent with $\mathrm{O}$ in a combination of aluminosilicates and quartz, as expected, based on the mineralogical analysis. 


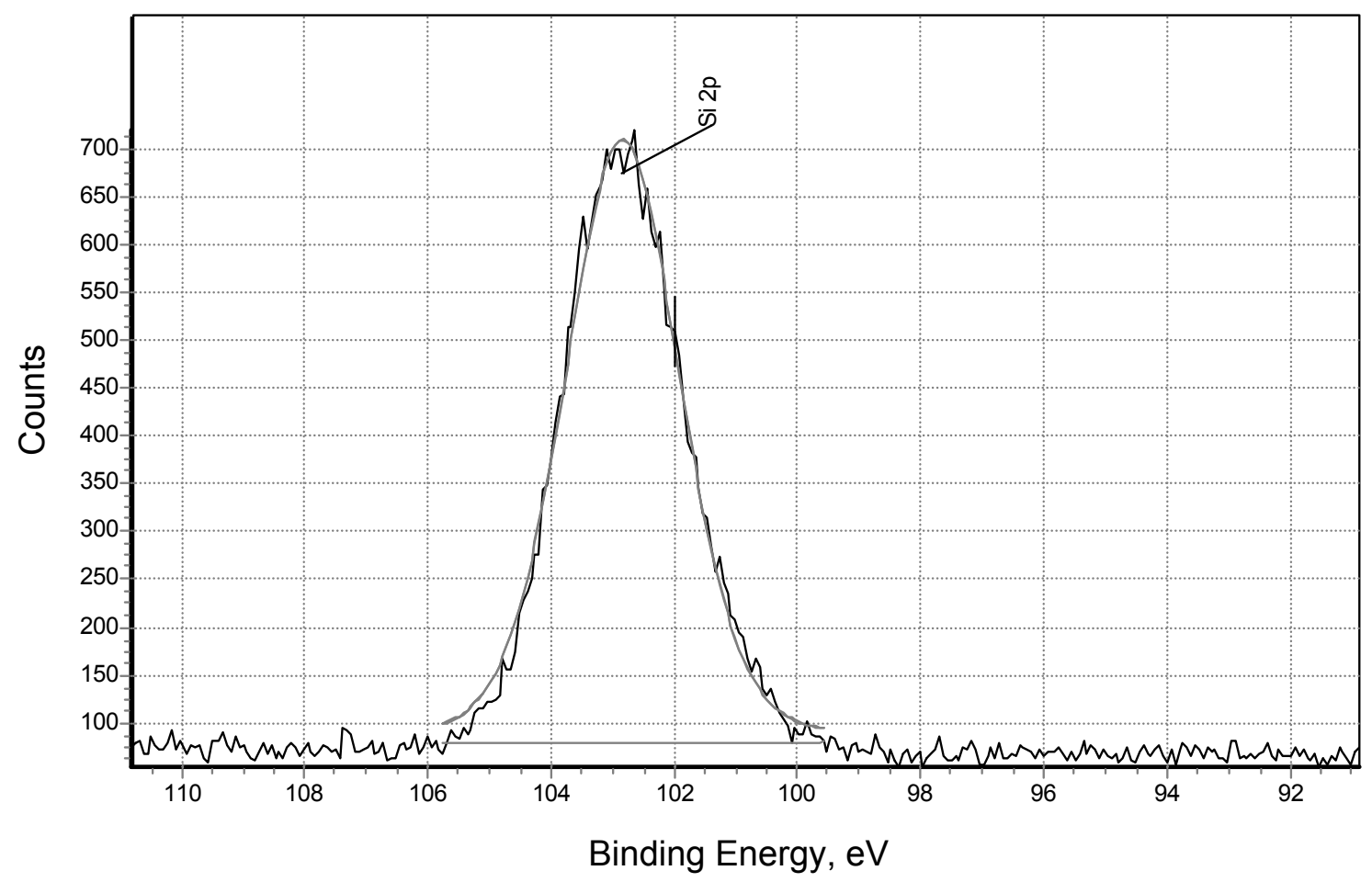

Figure 22. Typical Si 2p x-ray photoelectron spectrum in a Frenchman Flat powder sample.

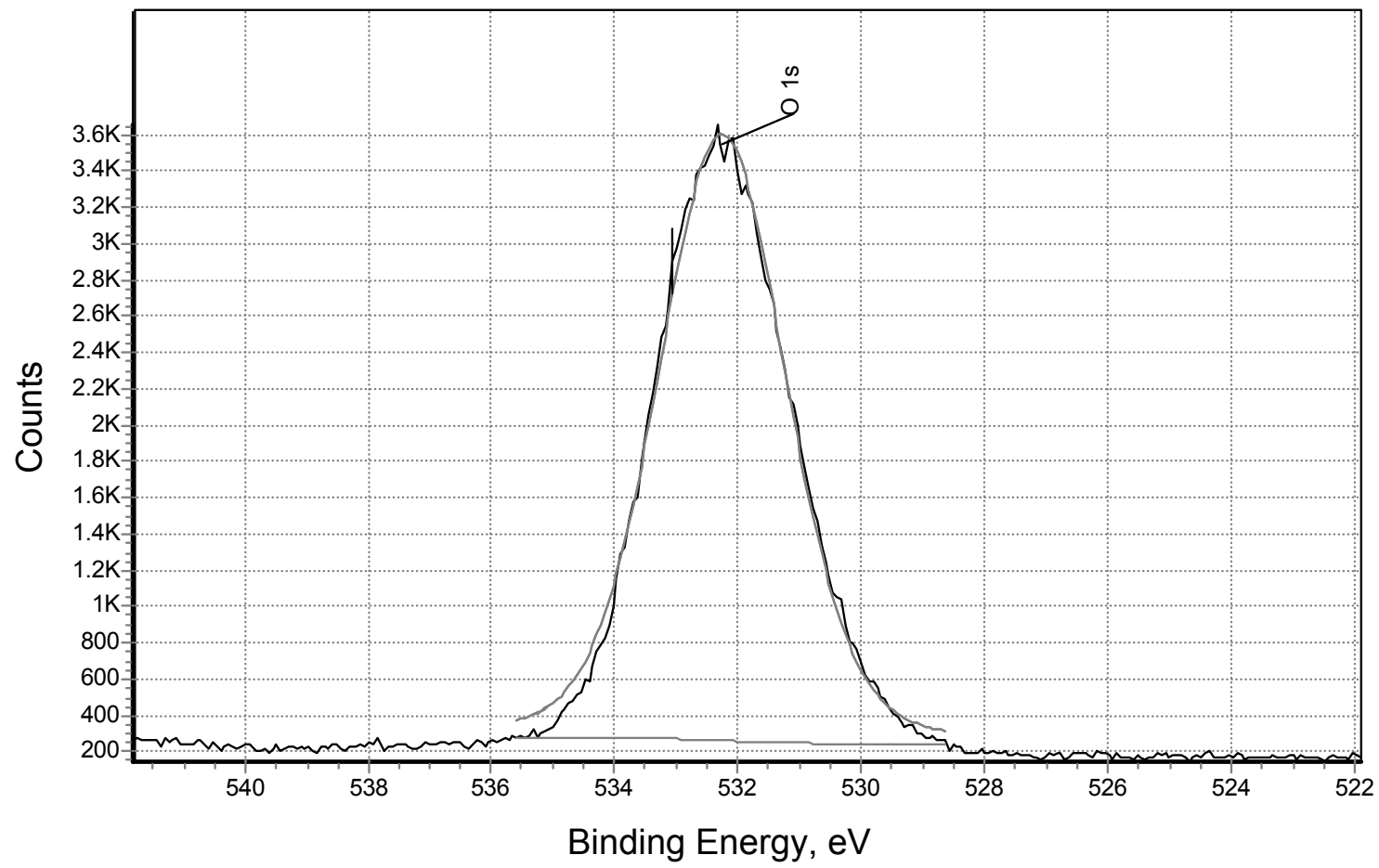

Figure 23. Typical O 1s x-ray photoelectron spectrum in a Frenchman Flat powder sample. 
The next set of major cations, in concentrations lower than the primary components $\mathrm{Al}$, $\mathrm{Si}$, and $\mathrm{O}$, included the cations of $\mathrm{Na}, \mathrm{K}$, and $\mathrm{Ca}$. Most elements were present in every sample. Potassium was present in every sample, whereas $\mathrm{Na}$ and $\mathrm{Ca}$ were not. A representative XPS spectrum of the $\mathrm{Na} 1 \mathrm{~s}$ peak is shown in Figure 24. Chemical shifts for these samples ranged between 0.41 and $1.25 \mathrm{eV}$, resulting in BEs mostly between 1072.4 and $1073.0 \mathrm{eV}$, with few exceptions. These results are certainly consistent with the presence of feldspars and other aluminosilicates. For example, the reported $\mathrm{BE}$ of $\mathrm{Na}$ in albite is $1072.2 \mathrm{eV}$ and values of $\mathrm{Na} 1 \mathrm{~s}$ BEs in molecular sieves range from 1071.8 to $1072.6 \mathrm{eV}$. The BEs for sodium carbonate and sodium bicarbonate appear to be lower, around $1071.5 \mathrm{eV}$.

A representative XPS spectrum of the $K 2 p$ peak is shown in Figure 25, where the spin orbit splitting of the $2 p$ level into $2 p_{1 / 2}$ and $2 p_{3 / 2}$ can be easily observed. The split orbit splitting was easily observed in all samples and the observed energy split was in good agreement with values reported in the literature. The chemical shift in the 2800D sample was consistently less than in the other two samples. Specifically, the average chemical shift in the 2800D sample was $0.02 \mathrm{eV}$, whereas the average chemical shifts in the 2960D and 3270D samples were -0.60 and -0.52 , respectively. The resulting average BEs were 294.02, 293.4, and 293.48 for samples 2800D, 2960D, and 3270D, respectively. These binding energies are common for a number of potassium salts, although no values for feldspars or zeolites have been reported specifically. It should be noted, however, that the differences in BEs for the three samples are consistent with the mineralogical analysis for these samples, namely the high fraction of zeolites in $2800 \mathrm{D}$ and the high fraction of feldspars in samples 2960D and 3270D. It is possible that the compositional differences could explain the differences in BEs for the three samples.

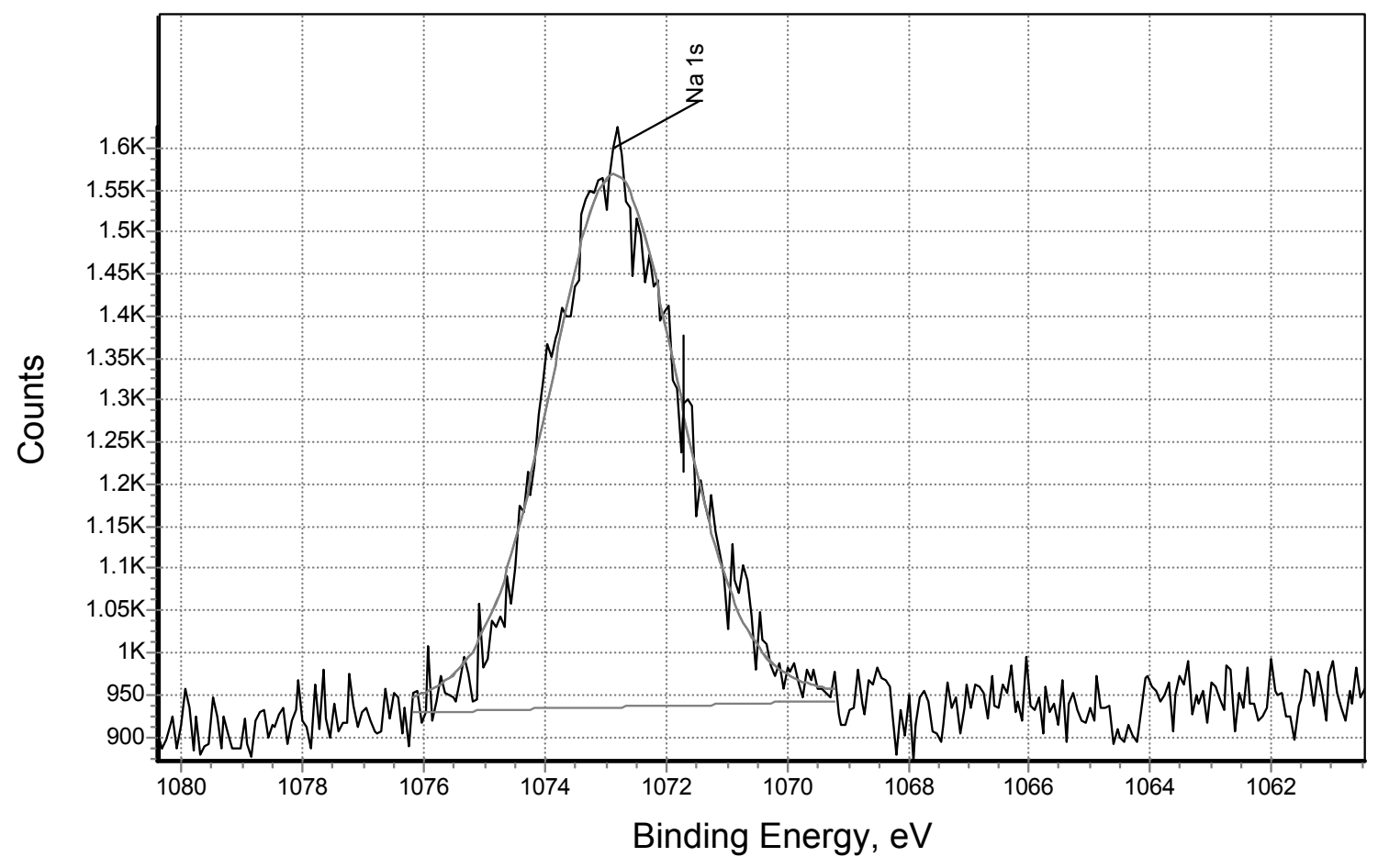

Figure 24. Typical Na 1s x-ray photoelectron spectrum in a Frenchman Flat powder sample. 


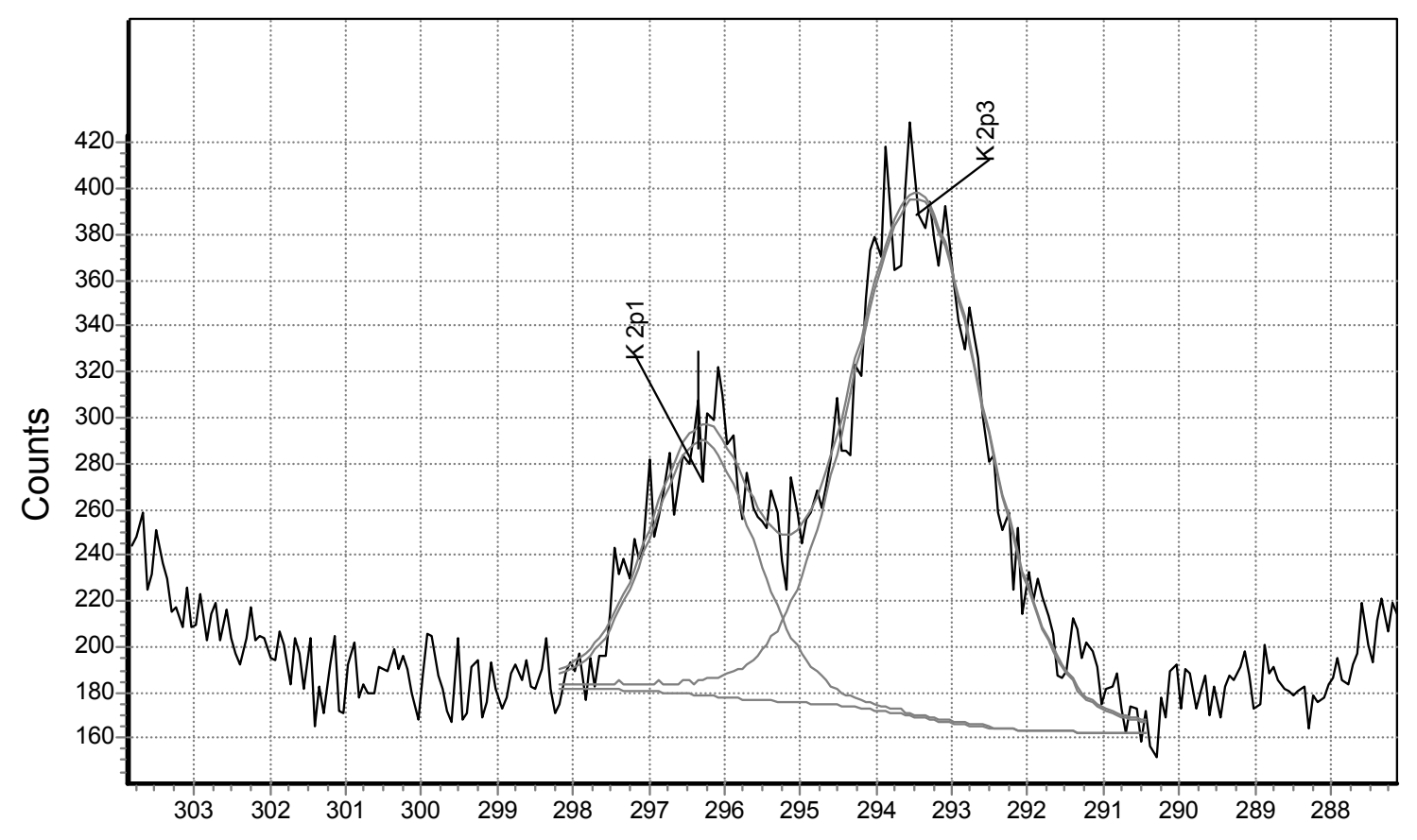

Binding Energy, eV

Figure 25. Typical K 2p x-ray photoelectron spectrum in a Frenchman Flat powder sample.

The concentration of $\mathrm{Ca}$ in the powder samples was typically lower than that of $\mathrm{Na}$ and $\mathrm{K}$. In some of the samples, Ca could not be detected. BE shifts ranged from 0.99 to $1.97 \mathrm{eV}$ with corresponding BEs between 347.99 and $348.87 \mathrm{eV}$. Typically, however, BE shifts ranged from 1.35 to $1.65 \mathrm{eV}$, resulting in BEs between 348.35 and $348.65 \mathrm{eV}$. There did not appear to exist a significant difference between the three different samples. A representative XPS spectrum of the $\mathrm{Ca} 2 \mathrm{p}$ peak is shown in Figure 26. The BEs measured appear to be too high for calcite (BE 346.9 $\mathrm{eV})$. The higher BE values measured would be consistent with aluminosilicates (e.g., feldspars) and calcium salts.

All samples had a relatively high Fe concentration, in several cases higher than the concentration of $\mathrm{Ca}$. With the exception of a single measurement of the $2800 \mathrm{D}$ powder, the chemical shifts ranged from 1.80 to $2.89 \mathrm{eV}$, resulting in BEs between 711.8 and $712.89 \mathrm{eV}$. A range of chemical shifts could be attributed to multiple sources of Fe, including smectite in all samples, mica in samples 2960D and 3270D, and hematite in sample 3270D. A representative XPS spectrum of the Fe 2p peak is shown in Figure 27. For comparison purposes, the reported Fe 2p BEs for hematite, ferrihydrite, and ferrous oxide are 710.9, 711.8, and 709.4, respectively. The Fe $2 p$ BEs obtained in this project appear to be on the high side of reported BEs. These results suggest that in these samples Fe must be present in a variety of coordination environments resulting in a range of BEs, as mentioned above.

A small quantity of $\mathrm{Sr}$ was detected on sample 2960D and a representative spectrum of the $\mathrm{Sr} 3 \mathrm{~d}$ photoelectric peak is shown in Figure 28. The BE of this peak in this sample was 134.77. The reported BE for $\mathrm{Sr}$ metal is $134.3 \mathrm{eV}$. Unfortunately, there are not as many values reported for different $\mathrm{Sr}$ environments, so that assigning of the peaks becomes more challenging. It appears though, that this value is too low for strontium oxide $(135.3 \mathrm{eV})$ and too high for strontium carbonate $(133.2 \mathrm{eV})$. These results, therefore, are not consistent with the formation of 


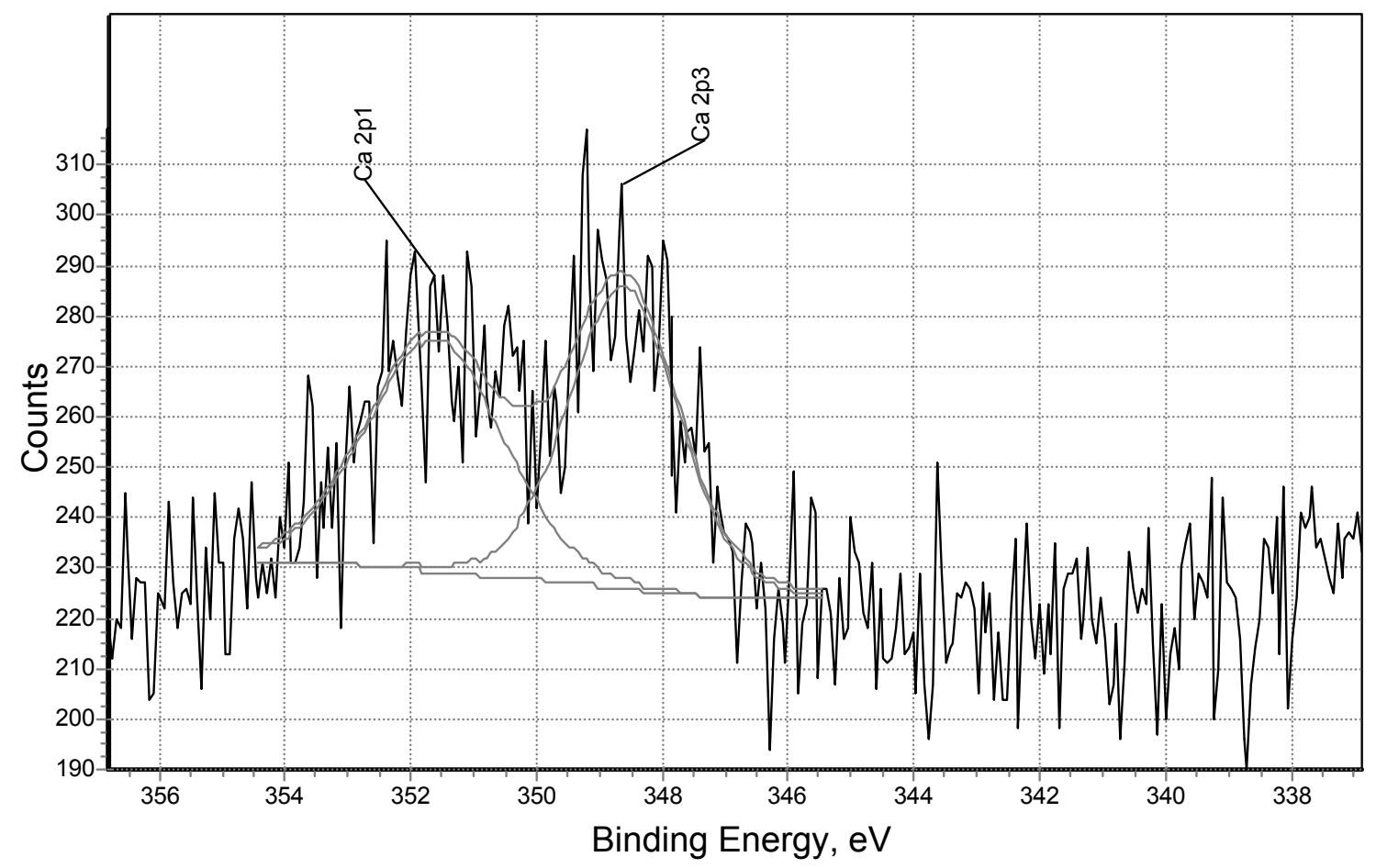

Figure 26. Typical Ca 2p x-ray photoelectron spectrum in a Frenchman Flat powder sample.

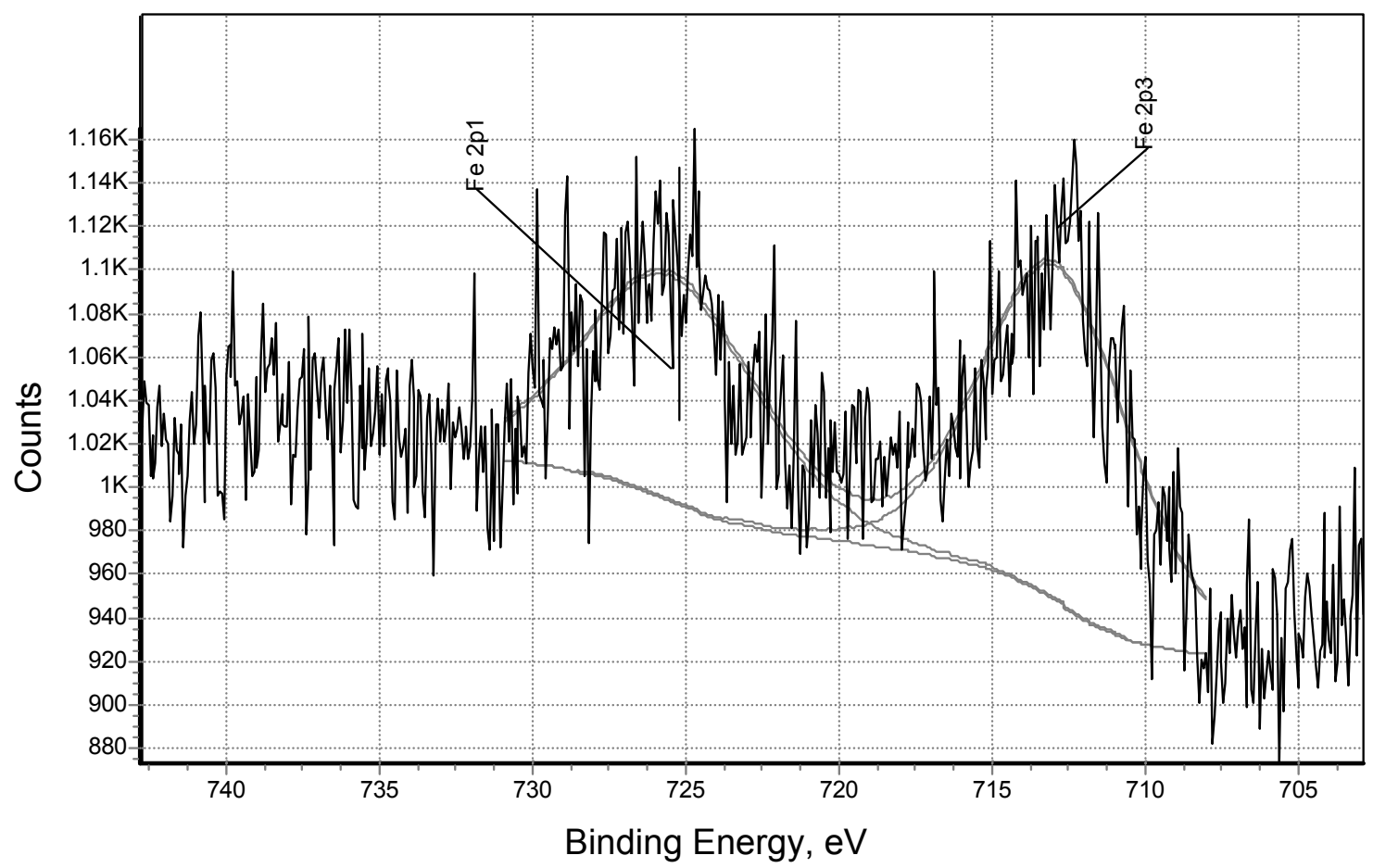

Figure 27. Typical Fe 2p x-ray photoelectron spectrum in a Frenchman Flat powder sample. 


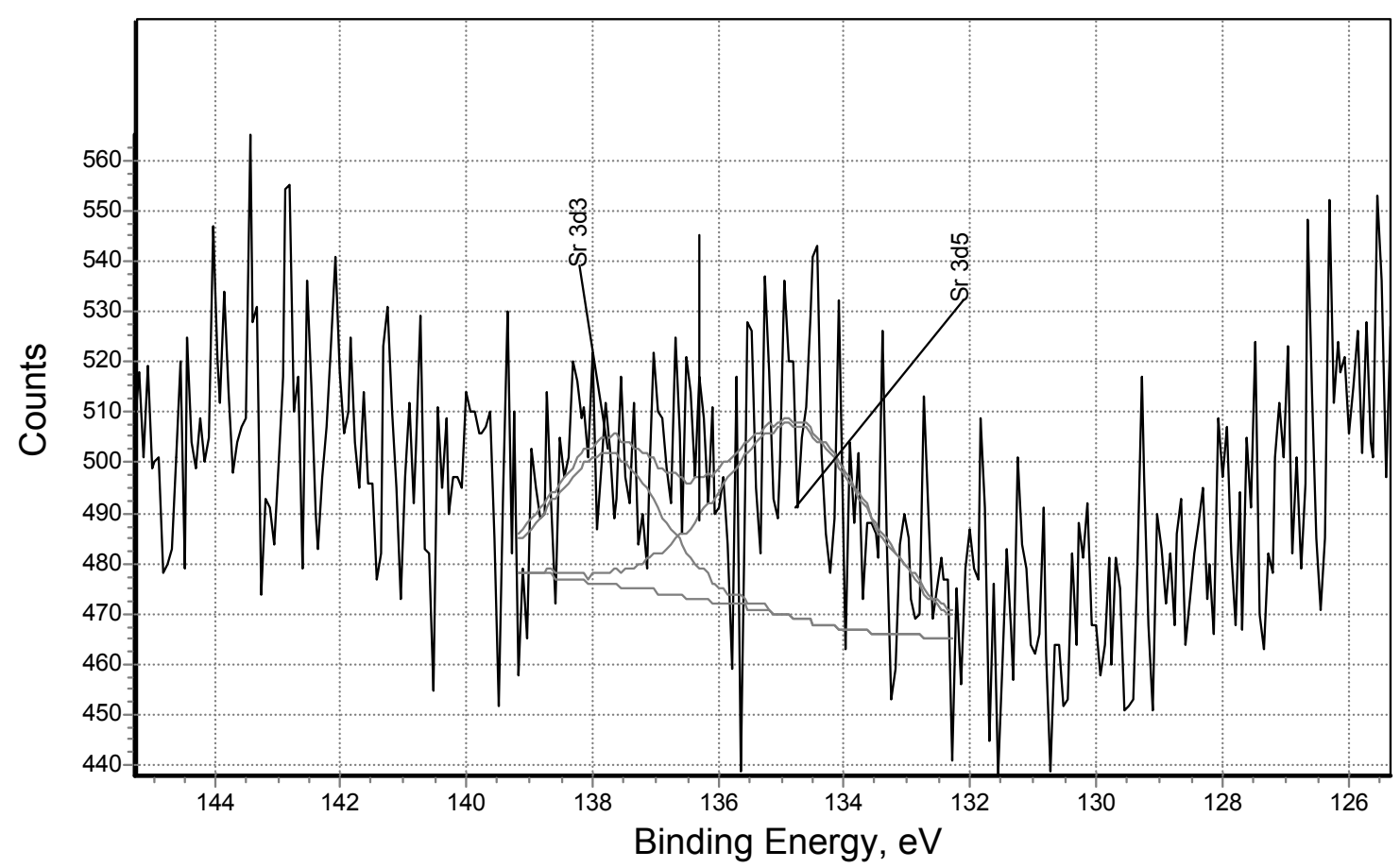

Figure 28. Typical Sr 3d x-ray photoelectron spectrum in a Frenchman Flat powder sorption sample.

either a strontium carbonate precipitate, or the formation of a simple $\mathrm{Sr}-\mathrm{O}$ bond, similar to the one in strontium oxide. This latter scenario would imply the formation of Sr inner-sphere complexes on these surfaces, against substantial evidence from spectroscopic studies that suggest that $\mathrm{Sr}$ forms outer-sphere complexes only. The measured BE, therefore, could be consistent with the presence of $\mathrm{Sr}$ as outer-sphere complexes.

Cesium was also detected on sample 2960D. A representative spectrum of the Cs $3 \mathrm{~d}$ photoelectric peak is shown in Figure 29. The measured chemical shift was $-0.76 \mathrm{eV}$, resulting in a BE of $725.24 \mathrm{eV}$. The reported BE for Cs is 726.0 or $726.4 \mathrm{eV}$. The measured values are therefore definitely lower than that and, most likely, correspond to a different chemical state. As for Sr, relatively few studies have been conducted that would help identify the chemical state in the sorption samples. The Cs $3 \mathrm{~d} \mathrm{BE}$ in cesium hydroxide, however, is reported as $724.5 \mathrm{eV}$, lower than the measured values. The measured BE for these sorption complexes, therefore, appears to be reasonable. 


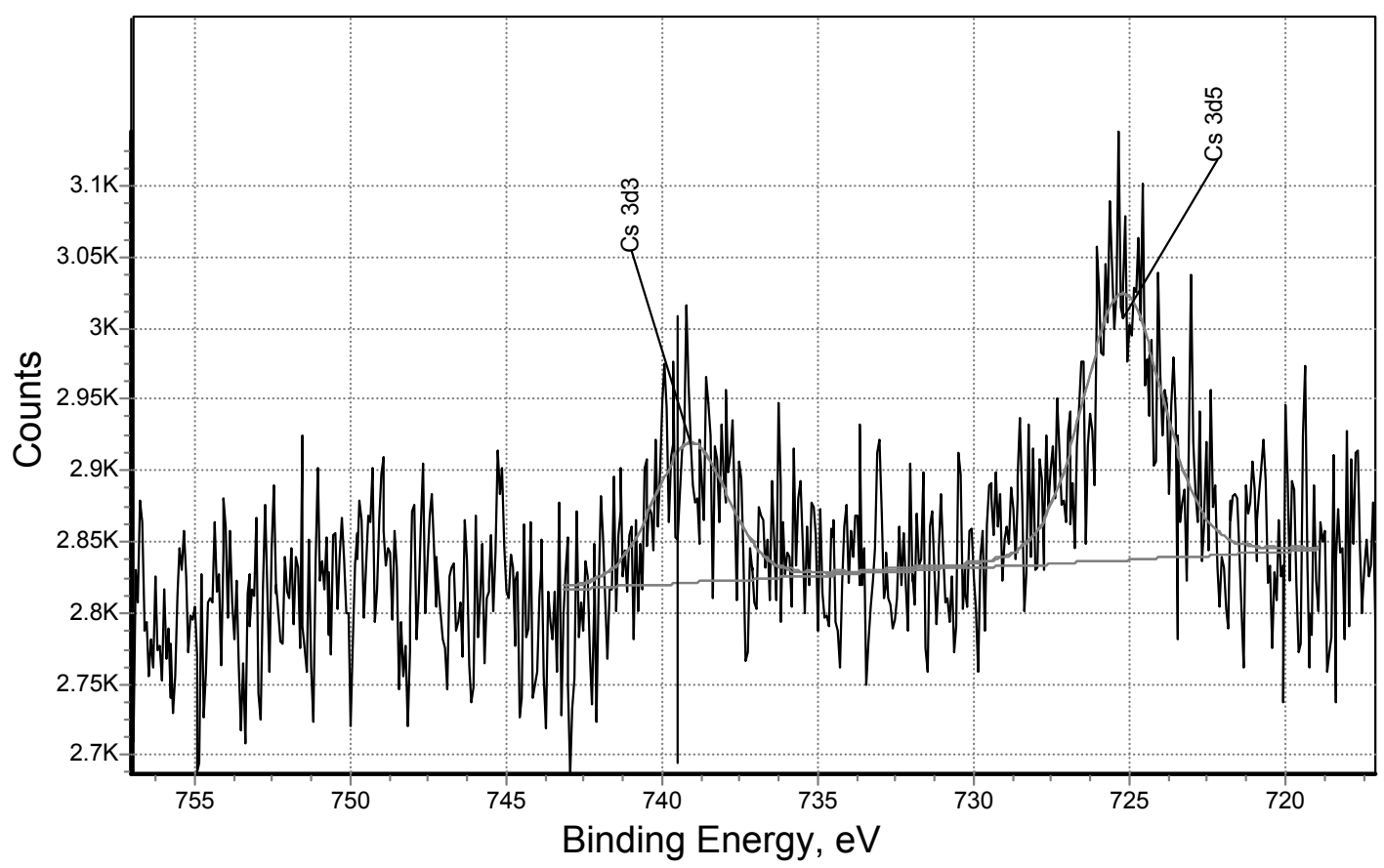

Figure 29. Typical Cs 3d x-ray photoelectron spectrum in a Frenchman Flat powder sorption sample.

\section{Surface Composition}

The composition of the powder samples was also determined using the XPS. The advantage of the XPS in elemental composition determination over other techniques, such as EDX, is that only the first few atomic layers contribute to the signal, thus allowing us to study the composition of the surfaces involved in sorption reactions. Sample analysis was conducted for the powder samples, both before and after the sorption reactions. In all cases, all major cations and $\mathrm{O}$ were included in the elemental composition table. The composition table is prepared automatically by the ESCAVB software by including the peaks of interest.

The calculations of surface composition are listed in Table 7 . In addition to the major cations and $\mathrm{O}$, elemental composition includes the $\mathrm{Cs}, \mathrm{Sr}$, and $\mathrm{Pb}$ adsorbates, where present. Although, strictly speaking, these elements were not present in the original samples and therefore it would not be appropriate to include them in the core composition, the total concentration of these elements is so small (approximately $0.1 \%$ ), so that the calculated percentage would not be affected by the inclusion of these elements.

The surface composition of the samples is reported as a percentage of atomic composition, based on the number of atoms present, as opposed to percentage composition by weight, as is sometimes done. The general trends in atomic composition will be summarized below. Oxygen, Si, and Al were, as expected, the most abundant elements at the surface, although in some measurements, Na concentration was almost as high as Al. Sodium and K were present at lower concentrations and in every sample. Calcium was not detectable in all samples. The ratio between $\mathrm{Na}, \mathrm{K}$, and $\mathrm{Ca}$ concentration was a function of the specific sample. The highest $\mathrm{Na}$ concentrations (approximately 3 to $5 \%$ ) were in sample $2800 \mathrm{D}$, the highest $\mathrm{K}$ concentrations (approximately 3.5\%) were in sample 2960D, and Ca concentrations were approximately the same in all samples, around 0.5 percent. Although Fe was present in all three 
Table 7. Atomic composition of powder samples.

\begin{tabular}{|c|c|c|c|c|c|c|c|c|}
\hline SAMPLE & \multicolumn{2}{|c|}{ Powder } & \multicolumn{2}{|c|}{ Powder } & \multicolumn{2}{|c|}{ Powder } & \multicolumn{2}{|c|}{ Powder } \\
\hline \multirow[t]{8}{*}{ ER5/3/2 2800D } & & & \multicolumn{2}{|c|}{ with $10 \mathrm{E}-5 \mathrm{M} \mathrm{Cs}$} & \multicolumn{2}{|c|}{ with $10 \mathrm{E}-5 \mathrm{M} \mathrm{Pb}$} & \multicolumn{2}{|c|}{ with $10 \mathrm{E}-5 \mathrm{M} \mathrm{Sr}$} \\
\hline & $\mathrm{Al} 2 \mathrm{p}$ & 4.48 & $\mathrm{Al} 2 \mathrm{p}$ & 5.02 & $\mathrm{Al} 2 \mathrm{p}$ & 1.50 & $\mathrm{Al} 2 \mathrm{p}$ & 4.29 \\
\hline & Si $2 p$ & 22.60 & Si $2 p$ & 24.11 & Si $2 p$ & 29.28 & Si $2 p$ & 26.42 \\
\hline & $\mathrm{O} 1 \mathrm{~s}$ & 64.71 & $\mathrm{O} 1 \mathrm{~s}$ & 64.43 & $\mathrm{O} 1 \mathrm{~s}$ & 68.20 & $\mathrm{O} 1 \mathrm{~s}$ & 64.24 \\
\hline & $\mathrm{Na} 1 \mathrm{~s}$ & 5.29 & $\mathrm{Na} 1 \mathrm{~s}$ & 4.25 & & & $\mathrm{Na} 1 \mathrm{~s}$ & 3.19 \\
\hline & $\mathrm{K} 2 \mathrm{p} 3$ & 1.27 & $\mathrm{~K} 2 \mathrm{p} 3$ & 1.24 & $\mathrm{~K} 2 \mathrm{p} 3$ & .449 & $\mathrm{~K} 2 \mathrm{p} 3$ & 1.03 \\
\hline & $\mathrm{Ca} 2 \mathrm{p}$ & 0.57 & $\mathrm{Ca} 2 \mathrm{p}$ & 0.49 & & & $\mathrm{Ca} 2 \mathrm{p}$ & 0.46 \\
\hline & $\mathrm{Fe} 2 \mathrm{p} 3$ & 1.08 & Fe $2 p 3$ & 0.46 & $\mathrm{Fe} 2 \mathrm{p} 3$ & .573 & Fe $2 p 3$ & 0.37 \\
\hline \multirow[t]{9}{*}{ ER5/3/2 2960D } & \multicolumn{2}{|c|}{ Powder } & \multicolumn{2}{|c|}{ Powder } & \multicolumn{2}{|c|}{$\begin{array}{c}\text { Powder } \\
\text { with } 10 \mathrm{E}-5 \mathrm{M} \mathrm{Pb}\end{array}$} & \multicolumn{2}{|c|}{$\begin{array}{c}\text { Powder } \\
\text { with } 10 \mathrm{E}-5 \mathrm{M} \mathrm{Sr}\end{array}$} \\
\hline & & & Cs $3 d 5$ & 0.13 & & $\operatorname{Sr} 3 d 5$ & 0.14 \\
\hline & $\mathrm{Al} 2 \mathrm{p}$ & 4.66 & $\mathrm{Al} 2 \mathrm{p}$ & 5.23 & $\mathrm{Al} 2 \mathrm{p}$ & 3.30 & $\mathrm{Al} 2 \mathrm{p}$ & 4.72 \\
\hline & Si $2 p$ & 23.10 & Si $2 p$ & 25.29 & Si $2 p$ & 28.45 & Si $2 p$ & 25.11 \\
\hline & $\mathrm{O} 1 \mathrm{~s}$ & 65.13 & $\mathrm{O} 1 \mathrm{~s}$ & 63.50 & $\mathrm{O} 1 \mathrm{~s}$ & 63.94 & $\mathrm{O} 1 \mathrm{~s}$ & 63.72 \\
\hline & $\mathrm{Na} 1 \mathrm{~s}$ & 3.19 & $\mathrm{Na} 1 \mathrm{~s}$ & 1.77 & $\mathrm{Na} 1 \mathrm{~s}$ & 0.86 & $\mathrm{Na} 1 \mathrm{~s}$ & 1.68 \\
\hline & $\mathrm{K} 2 \mathrm{p} 3$ & 3.45 & $\mathrm{~K} 2 \mathrm{p} 3$ & 3.29 & $\mathrm{~K} 2 \mathrm{p} 3$ & 2.94 & $\mathrm{~K} 2 \mathrm{p} 3$ & 3.43 \\
\hline & $\mathrm{Ca} 2 \mathrm{p} 3$ & 0.25 & $\mathrm{Ca} 2 \mathrm{p}$ & 0.47 & & & $\mathrm{Ca} 2 \mathrm{p}$ & 0.60 \\
\hline & $\mathrm{Fe} 2 \mathrm{p} 3$ & 0.22 & Fe $2 p 1$ & 0.33 & $\mathrm{Fe} 2 \mathrm{p} 3$ & 0.52 & Fe $2 p 3$ & 0.59 \\
\hline $\mathrm{ER} 5 / 3 / 23270 \mathrm{D}$ & \multicolumn{2}{|c|}{ Powder } & \multicolumn{2}{|c|}{$\begin{array}{c}\text { Powder } \\
\text { with } 10 \mathrm{E}-5 \mathrm{M} \mathrm{Cs}\end{array}$} & \multicolumn{2}{|c|}{$\begin{array}{c}\text { Powder } \\
\text { with } 10 \mathrm{E}-5 \mathrm{M} \mathrm{Pb}\end{array}$} & \multicolumn{2}{|c|}{$\begin{array}{c}\text { Powder } \\
\text { with } 10 \mathrm{E}-5 \mathrm{M} \mathrm{Sr}\end{array}$} \\
\hline & $\mathrm{Al} 2 \mathrm{p}$ & 4.64 & $\mathrm{Al} 2 \mathrm{p}$ & 5.34 & $\mathrm{Al} 2 \mathrm{p}$ & 3.37 & $\mathrm{Al} 2 \mathrm{p}$ & 4.20 \\
\hline & Si $2 p$ & 21.36 & Si $2 p$ & 23.40 & Si $2 p$ & 24.84 & Si $2 p$ & 22.27 \\
\hline & $\mathrm{O} 1 \mathrm{~s}$ & 67.44 & $\mathrm{O} 1 \mathrm{~s}$ & 66.06 & $\mathrm{O} 1 \mathrm{~s}$ & 68.09 & $\mathrm{O} 1 \mathrm{~s}$ & 66.57 \\
\hline & $\mathrm{Na} 1 \mathrm{~s}$ & 2.57 & $\mathrm{Na} 1 \mathrm{~s}$ & 0.91 & $\mathrm{Na} 1 \mathrm{~s}$ & 0.27 & $\mathrm{Na} 1 \mathrm{~s}$ & 3.14 \\
\hline & $\mathrm{K} 2 \mathrm{p} 3$ & 2.32 & $\mathrm{~K} 2 \mathrm{p} 3$ & 2.79 & $\mathrm{~K} 2 \mathrm{p} 3$ & 2.03 & $\mathrm{~K} 2 \mathrm{p} 3$ & 2.40 \\
\hline & $\mathrm{Ca} 2 \mathrm{p}$ & 0.44 & $\mathrm{Ca} 2 \mathrm{p}$ & 0.40 & $\mathrm{Ca} 2 \mathrm{p}$ & 0.27 & $\mathrm{Ca} 2 \mathrm{p}$ & 0.45 \\
\hline & Fe $2 p 3$ & 1.24 & $\mathrm{Fe} 2 \mathrm{p} 1$ & 1.10 & Fe $2 p 3$ & 1.12 & $\mathrm{Fe} 2 \mathrm{p} 3$ & 0.97 \\
\hline
\end{tabular}

samples, the highest concentration was found in sample $3270 \mathrm{D}$, approximately 1 percent, as expected, because of the presence of 1.1 percent hematite.

The atomic percentages of $\mathrm{O}, \mathrm{Si}$, and $\mathrm{Al}$ averaged around 65, 22, and 5 percent, respectively, accounting for approximately 92 percent of the sample. The highest $O$ percentage was approximately 68, the highest Si percentage was approximately 29, and the highest $\mathrm{Al}$ percentage was approximately 5 . These numbers are entirely consistent with the reported mineralogy of the samples that consisted mainly of zeolites, feldspars, quartz, mica, and smectites. In the case of quartz, the expected composition would be 33 percent $\mathrm{Si}$ and 67 percent $\mathrm{O}$, with no Al. In the case of feldspars, taking the mineral albite as an example with the formula 
$\mathrm{NaAlSi}_{3} \mathrm{O}_{8}$, the expected percentages would be $\mathrm{O}$ 62, Si 23, and $\mathrm{Al}$ and $\mathrm{Na} 8$ each. If half of $\mathrm{Na}$ were substituted by $\mathrm{K}$ the expected composition would have been $\mathrm{O} 62$, Si 23, A1 8, Na 4, and K 4. Assuming a mixture of 33 percent quartz and 67 percent feldspar would result in an approximate composition of 64 percent $\mathrm{O}, 26$ percent $\mathrm{Si}, 5$ percent $\mathrm{Al}, 3$ percent $\mathrm{Na}$, and 3 percent K.

This composition is indeed remarkably close to the obtained results and reassuring in terms of confidence in the XPS analytical results. In addition, these results are significant because they provide evidence that the surface composition is most likely very similar to the bulk mineralogy of the samples. It should be remembered that XRD is a bulk technique, unlikely to detect the presence of thin surface coatings. The presence of such coatings, however, can play an important role in surface controlled reactions. XPS is unique in that it allows us to examine the top surface layers of the sorbent. This type of analysis is even more important when rock is crushed, possibly creating new surfaces with different composition and therefore properties.

Estimation of Sorbent Surface Coverage by Cesium, Lead, and Strontium

One of the major advantages of the XPS technique is its ability to analyze the composition of a surface and, specifically, to determine the composition of the first few atomic monolayers of that surface. This is obviously a great advantage for the study of interfaces, including the study of sorption reactions, as these reactions are controlled by the interaction of dissolved ions with the uppermost layers of atoms on exposed surfaces. Based on macroscopic sorption experiments, one can determine the total uptake of a metal ion by a solid. This information, alone, however, cannot provide any insight into the sorption mechanism of ions of interest on mineral surfaces. For example, based on macroscopic information, one can determine the total amount of a metal sorbed onto a surface, but one cannot distinguish between sorption and surface precipitation. Such a distinction is particularly important when trying to distinguish between surface precipitation on one hand and diffusion followed by sorption on the other.

Using XPS, one can estimate the surface coverage of adsorbents, thereby obtaining a direct measurement of the distribution of metal ions at the surface of adsorbents. In this study, this was achieved by analyzing powder samples after they had been exposed to a metal ion solution. Because the formation of a surface precipitate is expected to result in multi-layer coverage of the surface and because the XPS signal coming from the uppermost layers is particularly amplified, a multi-layer coverage would be consistent with the formation of surface precipitates. In addition, it was thought that the different samples, with different mineralogical compositions, might result in a different coordination environment for the ions, thereby leading to different surface complexes that could be differentiated by XPS.

For this study, powder samples were analyzed by XPS and measured surface coverage estimates were compared to the estimated surface coverage based on macroscopic sorption experiments. The comparison between the three different metal cations was expected to provide information that could be used to distinguish between different sorption mechanisms. For the XPS analysis, sorption experiments were conducted as usual, except in larger, 250-mL containers to ensure adequate solid could be collected for analysis. Following equilibration, the samples were centrifuged, most of the supernatant was removed, and the remaining wet paste was freeze-dried using a Labconco freeze dryer. Freeze-drying was used to disturb the coordination environment of the sorbed metal ions as little as possible during the drying process. The samples were loaded onto aluminum sample holders and pumped down for at least one day in the prep chamber before they were introduced in the analytical chamber for surface analysis. 
The comparison of surface coverage as estimated by total metal uptake and measured by XPS involved a number of calculations and assumptions that will be briefly summarized here. The surface coverage based on total metal uptake was estimated by comparing the total amount of metal sorbed to the total number of surface sites available. The total amount of metal sorbed was estimated by the total initial metal concentration, the fractional metal uptake (percentage), and was converted to total number of atoms sorbed using Avogadro's number. The total number of sites was estimated from the solid concentration, the specific surface area of the solid, and an estimate of the surface site density of the sample. The highest uncertainty in these calculations stems from the estimate of the surface site density. A value of 5 sites $/ \mathrm{nm}^{2}$ was used for these calculations. This is an average value obtained from the literature and is only supposed to be an estimate. Obviously, this value would be a function of the mineral and is probably not constant even for a single solid. For different types of surface studies, researchers have used values ranging from 3 to 10 sites $/ \mathrm{nm}^{2}$. The value chosen, 5 sites $/ \mathrm{nm}^{2}$, was considered a reasonable average. Obviously, the calculated numbers depend on the choice for this parameter.

The estimation of surface coverage by XPS involved a number of assumptions. The adsorbed layer thickness was estimated by comparing the photoelectric peak of the metal of interest to the photoelectric peak of the most abundant cation on the surface, Si. Specifically, the adsorbed layer thickness was calculated based on Equation (2), a generalization of the equation used by Papelis et al. (1995) to calculate surface coverages of adsorbents.

$$
x=-\lambda_{\alpha} \cos \theta \ln \left(1-\frac{A_{x}}{A_{x}+A_{S i}}\right)
$$

where $\mathrm{x}$ is the adsorbed layer thickness $(\AA), \lambda_{\alpha}$ is the attenuation length $(\AA), \theta$ is the angle between the sample normal and the detector, and $A_{x}$ and $A_{S i}$ are the normalized areas of the metal ion of interest and Si, respectively. As explained in more detail in Papelis et al. (1995), the angle between the sample normal and the detector was assumed to be zero and the attenuation length was assumed to be $15 \AA$. The normalized area is calculated by the ESCAVB software and is a function of peak area, the number of scans collected, the peak photoionization cross section, a sensitivity exponent, and the position of the photoelectric peak. The percent monolayer coverage is then estimated by comparing the adsorbed layer thickness to an assumed monolayer thickness of $1.5 \AA$. For example a calculated adsorbed layer thickness of $0.5 \AA$ would be interpreted as 33 percent monolayer coverage.

The results of all calculations of surface coverage either by total metal uptake or as determined by XPS for $\mathrm{Pb}, \mathrm{Cs}$, and $\mathrm{Sr}$ are shown in Tables 8 through 10, respectively. As can be noticed by inspection of these tables, the elements of concern could not be detected in all three samples. In fact, Cs and Sr could be detected in sample 2960D only, while Pb could not be detected in any sample. Possible reasons for these results will be given below for the individual metals. 
Table 8. Comparison of percent monolayer coverage of Frenchman Flat powders as determined by total lead uptake and XPS.

\begin{tabular}{lccc}
\hline Samples & Percent Uptake & Surface Coverage by Total Uptake & Surface Coverage by XPS \\
\hline $2800 \mathrm{D}$ & 94.2 & 1.5 & ND \\
$2960 \mathrm{D}$ & 4.91 & 0.6 & ND \\
3270D & 2.71 & 0.2 & ND \\
\hline
\end{tabular}

ND: Not Detected

See text for assumptions and details

Table 9. Comparison of percent monolayer coverage of Frenchman Flat powders as determined by total cesium uptake and XPS.

\begin{tabular}{lccc}
\hline Samples & Percent Uptake & Surface Coverage by Total Uptake & Surface Coverage by XPS \\
\hline 2800D & 99.1 & 1.5 & ND \\
2960D & 56.47 & 20.0 & 4.96 \\
3270D & 58.46 & 3.5 & ND \\
\hline
\end{tabular}

ND: Not Detected

See text for assumptions and details

Table 10. Comparison of percent monolayer coverage of Frenchman Flat powders as determined by total strontium uptake and XPS.

\begin{tabular}{lccc}
\hline Samples & Percent Uptake & Surface Coverage by Total Uptake & Surface Coverage by XPS \\
\hline 2800D & 100.0 & 1.5 & ND \\
2960D & 8.9 & 3.2 & 5.75 \\
3270D & 33.32 & 2.0 & ND \\
\hline
\end{tabular}

ND: Not Detected

See text for assumptions and details

The comparison of percent monolayer coverage of all powders by $\mathrm{Pb}$ as determined by total uptake and XPS is shown in Table 8. As mentioned above and as can be seen in Table $8, \mathrm{~Pb}$ could not be determined in any of the samples. The results could be explained by considering the macroscopic sorption results and sample mineralogy. The total $\mathrm{Pb}$ concentration in all samples was $10^{-5} \mathrm{M}$. It was decided not to use any higher concentration to avoid the potential precipitation of $\mathrm{Pb}$. In the case of sample $2800 \mathrm{D}$, for example, because of the relatively high surface area, the resulting surface coverage was only 1.5 percent of a monolayer, although a fractional uptake of 94.2 percent was measured. This surface coverage is at or below the detection limit of the XPS measurements. If the entire $\mathrm{Pb}$ concentration were located at the surface of the particles, however, it could have been possible to detect $\mathrm{Pb}$ even under these low surface coverage conditions. It should be remembered, however, that essentially all sorption capacity of the $2800 \mathrm{D}$ sample was attributed to zeolites, primarily clinoptilolite. The sorption sites of these minerals are all internal and it is highly likely that the majority of $\mathrm{Pb}$ was beyond the top few monolayers that can be examined by XPS.

The surface coverage of the other samples, 2960D and 3270D, was even lower because the fractional uptake of $\mathrm{Pb}$ was 4.9 and 2.7 percent, respectively, resulting in surface coverage of 0.6 and 0.2 percent of a monolayer, respectively. These surface coverages are definitely beyond the detection limit of the technique and the fact that no $\mathrm{Pb}$ could be detected was certainly expected. The low $\mathrm{Pb}$ fractional uptake of these samples was definitely puzzling, given that based on the macroscopic sorption experiments, a much higher fractional uptake would have 
been expected under these conditions. The XPS results, however, were consistent with the measured uptake. In addition, the fact that $\mathrm{Pb}$ was not detectable by XPS is consistent with the absence of surface precipitation. If any precipitates were present, the effective surface coverage would have been substantially higher and $\mathrm{Pb}$ would have been detected.

The comparison of percent monolayer coverage of all powders by Cs as determined by total uptake and XPS is shown in Table 9. As can be seen in Table 9, only on sample 2960D could any Cs be detected. These results can be justified based on the sorption experiment results. Although the fractional Cs uptake was of the same order of magnitude for all three samples, 99, 56 , and 58 percent for the samples 2800D, 2960D, and 3270D, respectively, the differences in specific surface area and solid concentration used resulted in substantially different estimated surface coverages. For example, the estimated surface coverage of 2960D was 20 percent of a monolayer, whereas the surface coverage in samples $2800 \mathrm{D}$ and $3270 \mathrm{D}$ were much lower, 1.5 and 3.5 percent, respectively.

Given the low surface coverage of the 2800D sample and the zeolitic nature of the primary adsorbent, it is not surprising that no Cs could be detected. Similarly, although the surface coverage of the $3270 \mathrm{D}$ was higher $(3.5 \%)$ and could be expected to be detectable, it is possible that smectites were responsible for the majority of observed uptake. Similar to zeolites, it is possible that the effective detectable surface coverage decreases by sorption on porous materials.

Only on sample 2960D could Cs be detected and the corresponding Cs 3d peak is shown in Figure 29. The fact that Cs could be detected in this sample is not surprising, given the much higher surface coverage in this sample, 20 percent. If anything, the XPS measurements appear to underestimate surface coverage by a factor of 4 ( 5 versus $20 \%$ ). When accounting for the discrepancy, it should be remembered that these estimates are based on a number of assumptions with smaller or larger degree of uncertainty. The uncertainty in these parameters could easily account for the observed differences. In addition, the fact that most of Cs was probably associated with smectites and clinoptilolite, where most of the cation exchange capacity of this sample was, may have resulted in some shielding of Cs in deeper mineral layers, as explained above. At any rate, these results are also consistent with Cs sorption by ion exchange processes as opposed to sorption by surface precipitation.

The comparison of percent monolayer coverage of all samples by $\mathrm{Sr}$ as determined by total uptake and XPS is shown in Table 10. As in the case of Cs, Sr could only be detected on sample 2960D and the Sr 3d peak is shown in Figure 28. It will be noticed by inspection of Table 10 that the surface coverage on sample 2960D was higher compared to the other two samples. Specifically, surface coverage on sample 2960D was more than twice the surface coverage on sample $2800 \mathrm{D}$. In addition, the sorption on sample $2800 \mathrm{D}$ was most likely accounted for by sorption on internal zeolite sites, as explained above, and was therefore most likely difficult to quantify by XPS. The difference in surface coverage between samples 2960D and 3270D was not quite as significant but nevertheless present. Based on the results shown in Figure 28, it appears that a surface coverage of about 3 percent of a monolayer was close to the detection limit. It is therefore not surprising that a surface coverage almost half as much would not be detectable. It is also possible that differences in mineralogy of the samples would affect the limit of detection. The agreement between surface coverage as determined based on the macroscopic experiments and XPS, however, was within a factor of two and therefore considered satisfactory. As in the case of $\mathrm{Cs}$, these results point to the formation of Sr sorption complexes, as opposed to formation of surface precipitates. 
These results complement and help interpret the sorption and diffusion experiments and have significant implications for the migration of radionuclides in Frenchman Flat at the NTS. The surface coverages measured by XPS, whenever detectable, agree with formation of sorption complexes and not formation of surface precipitates. Even in the cases where elements of interest could not be determined, the absence of an XPS peak was either expected, based on the low surface coverage, or was consistent with sorption on internal sites of zeolites and smectites. These results provide therefore significant information regarding the mechanism of radionuclides of concern on aquifer materials from Frenchman Flat.

\section{SUMMARY AND CONCLUSIONS}

Sorption and desorption experiments were conducted with three different volcanic tuff samples from Frenchman Flat on the NTS. All these samples were obtained from well ER5/3/2 and from different depths. Specifically, the three samples were obtained from the following depths: 2800, 2960, and $3270 \mathrm{ft}$. Consequently, the three samples were referred to as ER5/3/2 2800D, ER5/3/2 2960D, and ER5/3/2 3270D. The letter D signifies that these samples represent in situ rock, according to the Los Alamos National Laboratory researchers who characterized the cuttings. For brevity, these samples are referred to as 2800D, 2960D, and 3270D, respectively.

The three samples of cuttings were crushed and characterized. Quantitative mineralogical characterization was performed by the Los Alamos National Laboratory. The specific surface area of the adsorbents was measured by nitrogen adsorption as a function of particle size. The bulk density and porosity of the cores was also determined, based on the nitrogen adsorption measurements. Finally, the composition of the surfaces, powders and pieces of cuttings, was detemined using x-ray photoelectron spectroscopy, in an attempt to focus specifically on the surfaces, where radionuclides interact with minerals and other aquifer materials.

The three samples had different physicochemical characteristics, which was why they were chosen. Sample 2800D, a mafic-poor Rainier Mesa nonwelded tuff, was mostly composed of zeolites (over $80 \%$, mostly clinoptilolite), feldspars (11\%), while the remaining sample was composed of opal, quartz, and smectite. The other two samples had much lower concentrations of zeolites. Sample 2960D, a crystal-rich Topopah Spring densely welded tuff, was composed primarily of feldspars $(71 \%)$ but it also included 4 percent clinoptilolite, while the remaining of the sample contained mostly quartz, smectite, mica, and hematite. Finally, sample 3270D, a Wahmonie Flat reworked tuff, was the only sample that did not contain any zeolites. The majority of this sample was composed of feldspars (64\%), while the remainder was composed of quartz $(18 \%)$, mica $(11 \%)$, smectite $(6 \%)$ and hematite $(1 \%)$.

Both macroscopic and spectroscopic experiments were conducted with the three samples. The macroscopic experiments included sorption and desorption experiments as well as diffusion of reactive tracers in larger particles. The reactive tracers used were $\mathrm{Sr}, \mathrm{Cs}$, and $\mathrm{Pb}$. Strontium

and Cs were used because ${ }^{90} \mathrm{Sr}$ and ${ }^{137} \mathrm{Cs}$ are radionuclides very commonly found in nuclear testing areas, in general, and the NTS, in particular. Lead was used because it is known to bind by different mechanism on oxide and hydroxide surfaces and it binds stronger than $\mathrm{Cs}$ or $\mathrm{Sr}$. Because of its strong binding affinity for surfaces, $\mathrm{Pb}$ could be used as an analog of strongly sorbing radionuclides, from a retardation point of view, even though its aqueous geochemistry may not necessarily be similar to the geochemistry of any actinides. Based on sorption data with different solid and metal concentrations, linear and Freundlich sorption isotherms were derived for sorption around $\mathrm{pH} \mathrm{8,} \mathrm{representing} \mathrm{a} \mathrm{common} \mathrm{NTS} \mathrm{groundwater} \mathrm{pH}$. Following sorption, desorption experiments were conducted to determine the reversibility of the sorption reactions. 
These experiments were conducted as a function of time to better understand kinetic aspects of the desorption processes. Based on the desorption experiments, linear desorption isotherms were also derived.

The sorption experiments can be summarized as follows. In general, the results were a function of both the metal ion of interest and the volcanic tuff sample. These differences were expected, based on the different aqueous chemistry of $\mathrm{Pb}, \mathrm{Cs}$, and $\mathrm{Sr}$ and the different mineralogical composition of the three samples. Lead was the most reactive of the three metals. The other two metals, Sr and Cs, exhibited similar behavior. Sorption of all metal ions on 2800D was the least $\mathrm{pH}$ dependent, as expected, given the high zeolite component in this sample. Sorption of $\mathrm{Cs}$ and $\mathrm{Sr}$ on all samples was minimally $\mathrm{pH}$ dependent, also expected, given that these ions are known to bind primarily on cation exchange sites of smectites and zeolites. The sorption of $\mathrm{Pb}$ on $3270 \mathrm{D}$, however, the sample with primarily amphoteric surface hydroxyl sites, was strongly $\mathrm{pH}$ dependent. Distribution coefficients, $K_{\mathrm{d}}$, for $\mathrm{Pb}$ ranged from $5.5 \times 10^{-3}$ to $1.29 \times 10^{-2} \mathrm{~m}^{3} \mathrm{~g}^{-1}$, while the $K_{\mathrm{d}} \mathrm{S}$ for $\mathrm{Cs}$ and $\mathrm{Sr}$ were approximately one order of magnitude lower. It appears, therefore, that under these conditions all three ions of concern would be retarded considerably.

The desorption experiments resulted in isotherms that were compared to the sorption isotherms. In all but one case, $K_{\mathrm{d}} \mathrm{s}$ obtained from desorption experiments were higher than the $K_{\mathrm{d}} \mathrm{s}$ obtained from sorption experiments. The differences were in several cases relatively small, approximately 20 to 25 percent, but in the case of sample $2800 \mathrm{D}$, the desorption $K_{\mathrm{d}} \mathrm{S}$ were higher than the sorption $K_{\mathrm{d}} \mathrm{s}$ by a factor of 3 to 4 . These differences are the result of the different mineralogies of the three samples. It appears that the higher the fraction of zeolites, the higher the irreversibility (or hysteresis) of sorption. Based on these experiments, sorption of $\mathrm{Pb}, \mathrm{Cs}$, and Sr would be largely reversible on aquifer materials from 2960D and 3270D, but not on aquifer materials from 2800D. Diffusion experiments were conducted with coarser particles, compared to the finer particles used in the sorption and desorption experiments. These experiments were conducted with $\mathrm{Cs}, \mathrm{Sr}$, and $\mathrm{Pb}$. The data were fitted with a pore diffusion model, assuming diffusion into a sphere from limited volume. The diffusion model was better at representing the uptake of cations by samples 2960D and 3270D compared to sample 2800D. This is because 2800D, the high zeolite concentration sample, with its high cation exchange capacity, resulted in fast quantitative removal of all three cations. The uptake of these cations by 2800D was therefore not controlled by a diffusion process and the model results were consistent with this hypothesis.

Lead uptake by any of the samples was also not very well modeled by the diffusion model. The reasons for the failure in the case of sample 2800D have already been mentioned. The reasons for failure in the other two samples are related to the mechanism responsible for $\mathrm{Pb}$ uptake by these solids. The combination of the tendency of $\mathrm{Pb}$ to form inner-sphere complexes and the relatively low surface coverage on these solids resulting in quantitative $\mathrm{Pb}$ removal points toward an instantaneous removal of $\mathrm{Pb}$ that is not controlled by diffusion into a porous matrix.

All powder samples were examined by x-ray photoelectron spectroscopy, before and after sorption experiments, to obtain additional information on surface composition and to help distinguish between different sorption mechanisms for the trace elements of interest. The elemental surface composition determined by XPS was consistent with the known sample mineralogy, namely a combination of zeolites, feldspars, smectites, mica, and quartz, with limited amount of hematite. In addition to the surface composition, XPS allowed us to distinguish between different chemical states of elements. The binding energies determined for 
the major cations and oxygen were consistent with expected binding energies in aluminosilicates. The binding energies for $\mathrm{Cs}$ and $\mathrm{Sr}$ were consistent with the expected coordination environment of these metals under these conditions, although additional studies would be required to establish a better database of binding energies as a function of coordination environment for these elements.

The surface coverage of adsorbents by $\mathrm{Cs}, \mathrm{Pb}$, and $\mathrm{Sr}$ was independently determined by XPS measurements following the sorption experiments. These surface coverages were compared to estimates from macroscopic uptake experiments. Surface coverages could only be determined for Cs and Sr sorption on 2960D. These results, however, were consistent with the estimated surface coverages based on the macroscopic experiments and point toward diffusion and sorption as a mechanism for cation sorption on these samples, as opposed to surface precipitate formation. In the cases where surface coverages could be estimated by XPS, there was good agreement between the value estimated by XPS and the surface coverage estimated from total metal uptake.

In summary, sorption, desorption, diffusion, and spectroscopic experiments with three different samples from Frenchman Flat were used to reduce the uncertainty of modeling interactions of radionuclides with mineral surfaces. These experiments clearly show the difference in degree of interaction between different metal ions with different aquifer materials. Sorption of all three cations was not entirely reversible and the stronger the sorption, the less reversible was the reaction. The reversibility of sorption was a function of both the sorbent mineral phase and the metal cation. The spectroscopic experiments allowed distinction between different possible sorption mechanisms and helped explained observations from macroscopic sorption experiments. Finally, this work showed the significance of aquifer material properties on the observed radionuclide transport behavior and the importance of a combination of studies to reduce transport modeling uncertainties.

These results have significant implications for the migration of radionuclides on the NTS.

- With respect to equilibrium sorption, all three elements could be considered as reactive or highly reactive. Lead showed the highest reactivity, followed by Cs, which was followed by Sr. Strontium in particular could be fairly mobile in rocks with high natural Sr content, although this was not the case in these samples.

- The sorption of radionuclides and other metal cations may not be entirely reversible. Based on these results, it appears that the higher the affinity of a metal cation for a specific surface, the higher the possibility for desorption hysteresis. Under these conditions, modeling the sorption of radionuclides as an equilibrium process could lead to errors.

- Based on the diffusion experiments, the time-dependent sorption of Cs and Sr could be interpreted as a diffusion-controlled process, whereas the sorption of strongly binding radionuclides may be considered instantaneous. Different sorption models (equilibrium versus kinetic) may therefore be more appropriate for the different radionuclides.

- The spectroscopic experiments were consistent with the equilibrium and diffusion experiments and can be used to choose appropriate sorption models. 


\section{REFERENCES}

Ames L.L., Jr., 1960. The cation sieve properties of clinoptilolite. Am. Mineral. 45:689-700.

Baes, C.F., Jr. and R.E. Mesmer, 1986. The Hydrolysis of Cations. Krieger, Malabar, FL, 489 pp.

Bargar, J.R., G.E. Brown Jr. and G.A. Parks, 1997. Surface complexation of Pb(II) at oxidewater interfaces: I. XAFS and bond-valence determination of mononuclear and polynuclear $\mathrm{Pb}$ (II) sorption products on aluminum oxides. Geochim. Cosmochim. Acta. 61, 2617-2637.

Biino, G.G. and P. Gröning, 1998. Cleavage mechanism and surface chemical characterization of phengitic muscovite and muscovite as constrained by X-ray photoelectron spectroscopy. Phys. Chem. Minerals, 25, 168-181.

Bohn, H.L., B.L. McNeal and G.A. O’Conner, 1985). Soil Chemistry. John Wiley and Sons, New York, $341 \mathrm{pp}$.

Briggs, D. and M.P. Seah, 1990. Practical Surface Analysis, Volume 1, Auger and X-ray Photoelectron Spectroscopy. John Wiley \& Sons, New York, 657 pp.

Brunauer S., P.H. Emmet and E. Teller, 1938. Adsorption of gases in multimolecular layers. J. Am. Chem. Soc. 60, 309-319.

Brundle, C.R. and A.D. Baker, 1978. Electron Spectroscopy: Theory, Techniques and Applications, 2. Academic Press, London, 287 pp.

Bryant, E.A., and J. Fabryka-Martin, 1991. Survey of hazardous materials used in nuclear testing. Report No. LA-12014-MS, Los Alamos, National Laboratory, Los Alamos, NM.

Chang R. (1988) Chemistry. $3^{\text {rd }}$ ed. McGraw-Hill, New York.

Chen, C.-C., C. Papelis and K.F. Hayes, 1998. Extended x-ray absorption fine structure (EXAFS) analysis of aqueous $\mathrm{Sr}^{\mathrm{II}}$ ion sorption at clay-water interfaces. In Sorption of Metals by Geomedia: Variables, Mechanisms, and Model Applications, (Edited by E.A. Jenne). Academic Press, San Diego, 333-348.

Davis J.A. and J.O. Leckie, 1980. Surface ionization and complexation at the oxide/water interface 3. Adsorption of anions. J. Colloid Interface Sci. 74, 32-43.

Drever, J.I., 1997. The Geochemistry of Natural Waters. $3^{\text {rd }}$ edition, Prentice Hall, 436 pp.

Faghihian, H., M.G. Marageh and H. Kazemian, 1999. The use of clinoptilolite and its sodium form for removal of radioactive cesium, and strontium from nuclear wastewater and $\mathrm{Pb}^{2+}$, $\mathrm{Ni}^{2+}, \mathrm{Cd}^{2+}, \mathrm{Ba}^{2+}$ from municipal wastewater. Applied Radiation Isotopes. 50:655-660.

Francis B.M., 1994. Toxic Substances in the Environment. John Wiley and Sons, New York, 360 pp.

Fuller C.C., J.A. Davis and G.A. Waychunas, 1993. Surface chemistry of ferrihydrite: Part 2. Kinetics of arsenate adsorption and coprecipitation, Geochim. Cosmochim. Acta. 57, 22712282.

Gregg S.J. and K.S.W. Sing, 1982. Adsorption, Surface Area, and Porosity. $2^{\text {nd }}$ ed., Orlando, Florida, Academic Press, Inc., 303 pp. 
Haggerty, G.M. and R.S. Bowman, 1994. Sorption of chromate and other inorganic anions by organo-zeolite. Environ. Sci. Technol. 28, 452-458.

Hammond, C.R., 1995. The Elements. In CRC Handbook of Chemistry and Physics. $80^{\text {th }}$ ed. (ed. David R. Lide). New York, NY, CRC Press, 4.1-4.34.

Hayes, K.F., A.L. Roe, G.E. Brown, Jr., K.O. Hodgson, J.O. Leckie, and G.A. Parks, 1987. In situ X-ray absorption study of surface complexes: selenium oxyanions on $\alpha-\mathrm{FeOOH}$. Science. 238, 783.

Hayes, K.F. and J.O. Leckie. 1987. Modeling ionic strength effects on cation adsorption at hydrous oxide/solution interfaces. J. Colloid Interface Sci. 115, 564.

Hayes, K.F., C. Papelis and J.O. Leckie, 1988. Modeling ionic strength effects on anion adsorption at hydrous oxide/solution interfaces. J. Colloid Interface Sci. 125, 717-726.

Hingston, F.J., A.M. Posner and J.P. Quirk, 1972. Anion adsorption by goethite and gibbsite. I. The role of the proton in determining adsorption envelopes. J. Soil Sci. 23, 177-192.

Hochella, M.F., Jr., 1988. Auger electron and X-ray photoelectron spectroscopies. In Spectroscopic Methods in Mineralogy and Geology (edited by F.C. Hawthorne), Reviews in Mineralogy. 18, 573-637.

Hochella, M.F., Jr., 1990. Atomic structure, microtopography, compound and reactivity of mineral surfaces. In Mineral-Water Interface Geochemistry (edited by M.F. Hochella, Jr. and A.F. White), Reviews in Mineralogy. 23, 87-132.

Hogan, R.G., 1993. Selenium. In Handbook of Hazardous Materials (ed. Morton Corn). San Diego, California, Academic press, 649-660.

Inglezakis, V.J., M.D. Loizidou and H.P. Grigoropoulou, 2002. Equilibrium and kinetic ion exchange studies of $\mathrm{Pb}^{2+}, \mathrm{Cr}^{3+}, \mathrm{Fe}^{3+}$ and $\mathrm{Cu}^{2+}$ on natural clinoptilolite, Water Resour. Res. 36, 2784-2792.

Jaffe, H.W., 1988. Introduction to Crystal Chemistry. Cambridge University Press, Cambridge, $161 \mathrm{pp}$.

Katz, L.E. and K.F. Hayes, 1995. Surface complexation modeling: I. Strategy for modeling monomer complex formation at moderate surface coverage. J. Colloid Interface Sci. 170, 477-490.

Kelly, M.A., 1986. Electron spectroscopy for chemical analysis. In Encyclopedia of Materials Science and Engineering, (edited by M.B. Bever), No. 2. Pergamon Press, Oxford, 14621467.

Langella, A., M. Pansini, P. Cappelletti, B. de Gennaro, M. de' Gennaro and C. Colella, 2000. $\mathrm{NH}_{4}{ }^{+}, \mathrm{Cu}^{2+}, \mathrm{Zn}^{2+}, \mathrm{Cd}^{2+}$ and $\mathrm{Pb}^{2+}$ exchange for $\mathrm{Na}^{+}$in a sedimentary clinoptilolite, Narth Sardinia, Italy, Microporous Mesoporous Mater. 37, 337-343.

McBride, M.B., 1994. Environmental Chemistry of Soils. Oxford University Press, New York, $406 \mathrm{pp}$.

Neretnieks, I., 1980. Diffusion in the rock matrix: An important factor in radionuclide retardation. J. Geophys. Res., 85, 4379-4397. 
O’Day, P.A., G.E. Brown, Jr. and G.A. Parks, 1994a. X-ray absorption spectroscopy of cobalt(II) multinuclear surface complexes and surface precipitates on kaolinite. J. Colloid Interface Sci. $165,269-289$.

O’Day, P.A., G.E. Brown, Jr., and G.A. Parks, 1994b. Molecular structure and binding sites of cobalt(II) surface complexes on kaolinite from x-ray absorption spectroscopy. Clays \& Clay Minerals. 42, 337-355.

Papelis, C., G.E. Brown, G.A. Parks and J.O. Leckie, 1995. X-ray absorption spectroscopic studies of cadmium and selenite adsorption on aluminum oxides. Langmuir 11, No.6, 20412048.

Papelis, C. and K.F. Hayes, 1996. Distinguishing between interlayer and external sorption sites of clay minerals using x-ray absorption spectroscopy. Colloids and Surfaces, A. 107, 89-96.

Perry, D.L., J.A. Taylor and C.D. Wagner, 1990. X-ray induced photoelectron and Auger spectroscopy. In Instrumental Surface Analysis of Geologic Materials (edited by D.L. Perry), VCH Publishers, New York, 45-86.

Rajec, P., F. Macasek, M. Feder, P. Misaelides and E. Samajova, 1998. Sorption of caesium and strontium on clinoptilolite- and mordenite-containing sedimentary rocks, J. Radioanal. Nucl. Chem. 229, 49-56.

Sax, N.I., 1981. Cancer Causing Chemical. Van Nostrand Reinhold, New York, 466 pp.

Scofield, J.H., 1976. Hartree-Slater subshell photoionization cross sections at 1254 and $1487 \mathrm{eV}$. J. Electron Spectr. Rel. Phen. 8, 129-137.

Shackelford, C.D., 1991. Laboratory diffusion testing for waste disposal - A review. J. Contam. Hydrol. 7, 177-217.

Skagius, K. and I. Neretnieks, 1986. Porosities and diffusivities of some nonsorbing species in crystalline rocks. Water Resour. Res. 22, 389-398.

Sloop, D.A., 1998. Equilibrium studies of ion sorption on zeolitized tuff from Rainier Mesa, Nye County, Nevada. M.S. thesis, Dept. of Geoscience, University of Nevada, Las Vegas.

Squibb, K.S. and E.T. Snow, 1993. Chromium. In Handbook of Hazardous Materials (ed. Morton Corn). San Diego, California, Academic Press, 127-144.

Stumm, W., R. Kummert and L. Sigg, 1980. A ligand exchange model for the adsorption of inorganic and organic ligands at hydrous oxide interfaces. Croat. Chem. Acta. 53, 291-312.

Tachi, Y., T. Shibutani, H. Sato and M. Yui, 1998. Sorption and diffusion behavior of selenium in tuff. J. Contam. Hydrol. 35, 77-89.

Travis C.C. and E.L. Etnier, 1981. A survey of sorption relationships for reactive solutes in soil. J. Environ. Qual. 10(1), 8-17.

Turner, N.H., 1992. Estimates of peak areas and relative atomic amounts from wide-scan XPS spectra. Surf. Interface Anal. 18, 47-51.

Um, W., 2001. Sorption mechanisms and transport behavior of $\mathrm{Sr}$ (II) and $\mathrm{Pb}$ (II0 on zeolitized tuffs from the Nevada Test Site. Ph.D. Dissertation, Graduate Program of Hydrologic Science. University of Nevada, Reno. 
Um, W. and C. Papelis, 2003. Sorption mechanisms of Sr(II) and $\mathrm{Pb}(\mathrm{II})$ on zeolitized tuffs from the Nevada Test Site as a function of $\mathrm{pH}$ and ionic strength. Submitted to American Mineralogist.

U.S. Department of Energy, Nevada Operations Office, 2000. United States Nuclear Tests, July 1945 Through September 1992. DOE/NV-209, Las Vegas, NV, 182 pp.

Webb, P.A. and C. Orr, 1997. Analytical methods in fine particle technology. Micromeritics Instrument Corporation, Norcross, Georgia, 301pp.

Weber, W.J., Jr. and F.A. DiGiano (1996). Process Dynamics in Environmental Systems. Environmental Science and Technology. John Wiley and Sons, New York, 943 pp. 


\section{DISTRIBUTION}

Jim Aldrich

Los Alamos National Laboratory

P.O. 1663, M/S D-462

Los Alamos, NM 87545

Bob Bangerter

Environmental Restoration Division

Nevada Site Office

National Nuclear Security Administration

U.S. Department of Energy

P.O. Box 98518

Las Vegas, NV 89193-8518

Rick Betteridge, Director

Technology Division

Nevada Site Office

National Nuclear Security Administration

U.S. Department of Energy

P.O. Box 98518

Las Vegas, NV 89193-8518

Melody Bell

Office of Business Affairs

Nevada Site Office

National Nuclear Security Administration

U.S. Department of Energy

P.O. Box 98518

Las Vegas, NV 89193-8518

Jeff Daniels

Lawrence Livermore National Laboratory

P.O. Box 808

M/S J514 CST-7

Livermore, CA 94551

Barbara Deshler

Shaw Environmental, Inc.

7710 W. Cheyenne

Las Vegas, NV 89129

Dave Finnegan

Los Alamos National Laboratory

P.O. Box 1663, M/S J-514, CST-7

Los Alamos, NM 87545
Ward Hawkins

Los Alamos National Laboratory

P.O. Box 1663, M/S F-665

Los Alamos, NM 87545

Bruce Hurley

Hydrology Program Manager

Environment, Safety \& Health Division

Nevada Site Office

National Nuclear Security Administration

U.S. Department of Energy

P.O. Box 98518

Las Vegas, NV 89193-8518

Kenneth Hoar, Director

Environment, Safety \& Health Division

Nevada Site Office

National Nuclear Security Administration

U.S. Department of Energy

P.O. Box 98518

Las Vegas, NV 89193-8518

Marjory Jones

Division of Hydrologic Sciences

Desert Research Institute

2215 Ragio Parkway

Reno, NV 89512-1095

Randy Laczniak

Water Resources Division

U.S. Geological Survey

6770 S. Paradise Rd.

Las Vegas, NV 89119

Dirk Schmidhofer

Stockpile Stewardship Division

Nevada Site Office

National Nuclear Security Administration

U.S. Department of Energy

P.O. Box 98518

Las Vegas, NV 89193-8518

Ken Ortego

Bechtel Nevada

P.O. Box 98521

Las Vegas, NV 89193-8521 
Gayle Pawloski

Lawrence Livermore National Laboratory

P.O. Box 808 L-221

Livermore, CA 94551

Ken Rehfeldt

HSI-GeoTrans

7710 W. Cheyenne

Las Vegas, NV 89129

Tim Rose

Lawrence Livermore National Laboratory

P.O. Box 808, M/S L-231

Livermore, CA 94551

Chuck Russell

Division of Hydrologic Sciences

Desert Research Institute

755 E. Flamingo Road

Las Vegas, NV 89119-7363

David Shafer

Division of Hydrologic Sciences

Desert Research Institute

755 E. Flamingo Road

Las Vegas, NV 89119-7363

Bonnie Thompson

Water Resources Division

U.S. Geological Survey

6770 S. Paradise Rd.

Las Vegas, NV 89119

Rick Waddell

HSI-GeoTrans

9101 Harlan Street, Ste. 210

Westminster, CO 80030

Janet Wille

Shaw Environmental, Inc.

7710 W. Cheyenne

Las Vegas, NV 89129

Nevada State Library and Archives

State Publications

100 North Stewart Street

Carson City, NV 89701-4285

Archives

Getchell Library

University of Nevada, Reno
DeLaMare Library/262

University of Nevada, Reno

Document Section, Library

University of Nevada, Las Vegas

4505 Maryland Parkway

Las Vegas, NV 89154

Shaw Environmental, Inc.

P.O. Box 93838

Bldg. B-1, M/S 439

Las Vegas, NV 89193-3838

ATTN: Toni Miller, M/S 439

Library

Southern Nevada Science Center

Desert Research Institute

755 E. Flaming Road

Las Vegas, NV 89119-7363

Public Reading Facility

Nevada Site Office

National Nuclear Security Administration

U.S. Department of Energy

P.O. Box 98521

Las Vegas, NV 89193-8521

Technical Library

Nevada Site Office

National Nuclear Security Administration

U.S. Department of Energy

P.O. Box 98518

Las Vegas, NV 89193-8518

Office of Scientific and Technical Information

U.S. Department of Energy

P.O. Box 62

Oak Ridge, TN 37831-9939

(electronic copy) 